C13.44:74

NBS MONOGRAPH 74

Creep and Drying Shrinkage

of Lightweight and

Normal-Weight Concretes

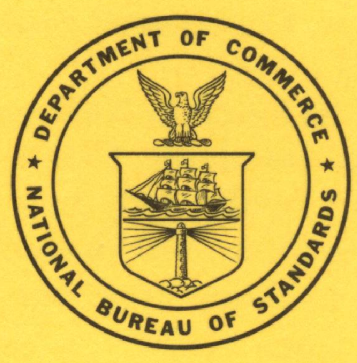

U.S. DEPARTMENT OF COMMERCE

NATIONAL BUREAU OF STANDARDS 


\section{THE NATIONAL BUREAU OF STANDARDS}

\section{Functions and Activities}

The functions of the National Bureau of Standards include the development and maintenance of the national standards of measurement and the provision of means and methods for making measurements consistent with these standards; the determination of physical constants and properties of materials; the development of methods and instruments for testing materials, devices, and structures; advisory services to government agencies on scientific and technical problems; invention and development of devices to serve special needs of the Government; and the development of standard practices, codes, and specifications, including assistance to industry, business and consumers in the development and acceptance of commercial standards and simplified trade practice recommendations. The work includes basic and applied research, development, engineering, instrumentation, testing, evaluation, calibration services, and various consultation and information services. Research projects are also performed for other government agencies when the work relates to and supplements the basic program of the Bureau or when the Bureau's unique competence is required. The scope of activities is suggested by the listing of divisions and sections on the inside of the back cover.

\section{Publications}

The results of the Bureau's research are published either in the Bureau's own series of publications or in the journals of professional and scientific societies. The Bureau itself publishes three periodicals available from the Government Printing Office: The Journal of Research, published in four separate sections, presents complete scientific and technical papers; the Technical News Bulletin presents summary and preliminary report on work in progress; and Central Radio Propagation Laboratory Ionospheric Predictions provides data for determining the best frequencies to use for radio communications throughout the world. There are also seven series of nonperiodical publications: Monographs, Applied Mathematics Series, Handbooks, Miscellaneous Publications, Technical Notes, Commercial Standards, and Simplified Practice Recommendations.

A complete listing of the Bureau's publications can be found in National Bureau of Standards Circular 460, Publications of the National Bureau of Standards, 1901 to June 1947 (\$1.25), and the Supplement to National Bureau of Standards Circular 460, July 1947 to June 1957 (\$1.50), and Miscellaneous Publication 240, July 1957 to June 1960 (includes Titles of Papers Published in Outside Journals 1950 to 1959) (\$2.25); available from the Superintendent of Documents, Government Printing Office, Washington, D.C., 20402. 
UNITED STATES DEPARTMENT OF COMMERCE • Luther H. Hodges, Secretary NATIONAL BUREAU OF STANDARDS • $\quad \Lambda$. I. Astin, Director

\section{Creep and Drying Shrinkage of Lightweight and Normal-Weight Concretes}

T. W. Reichard

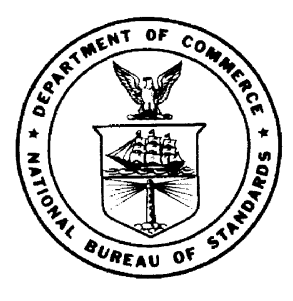

National Bureau of Standards Monograph 74 Issued March 4, 1964

For sale by the Superintendent of Documents, U.S. Government Printing Office Washington, D.C., 20402 - Price 30 cents 
Library of Congress Catalog Card Number: 64-60007 


\section{Contents}

2. Scope of investigation...

3. Materials _...

3.1. Aggregates

3.1.1. Lightweight aggregates...

3.1.2. Natural dense aggregates $\ldots \ldots \ldots \ldots$

3.3. Air-entraining agent

4. Concrete mixes

4.1. Trial mixes

4.2. Test concrete mixes

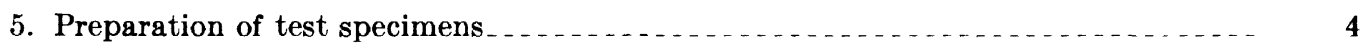

5.1. Description of specimens

5.3. Curing of the series A concretes.

5.4. Preparation of cylinder bearing surfaces

6. Test procedure $\ldots \ldots \ldots \ldots$

6.1. Compressive strength test $\ldots \ldots \ldots \ldots \ldots$

6.2. Dynamic modulus of elasticity and Poisson's ratio

6.4. Creep and shrinkage text.

7. Results $\ldots \ldots \ldots \ldots \ldots \ldots \ldots$

7.1. Results-Series A tests_.

7.1.1. Compressive strength test results

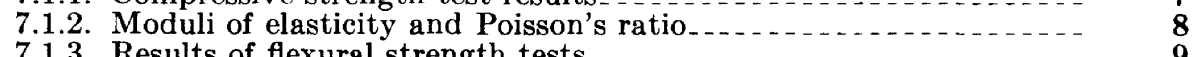

7.1.3. Results of flexural strength tests

7.1.5. Moisture expansion

7.2. Results-Series B tests_._.

7.2.1. Supplemental test I

7.2.2. Supplemental test II

7.2.3. Supplemental test III $\ldots \ldots \ldots \ldots$

7.2.4. Supplemental test IV

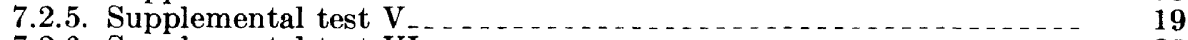

7.2.6. Supplemental test VI

8. Discussion of results $\ldots \ldots \ldots \ldots \ldots \ldots$

8.1. Significance of slump as a measure of water content of concrete

8.2. Statistical analyses of test results . .

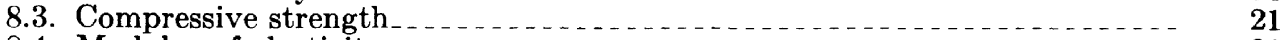

8.4. Modulus of elasticity

8.5. Flexural strength

8.6. Creep and shrinkage

8.7. Conclusions $\ldots \ldots \ldots$

9. Appendix _..... .

9.1. Lightweight aggregates

9.2. Strain measurements $\ldots \ldots \ldots \ldots$

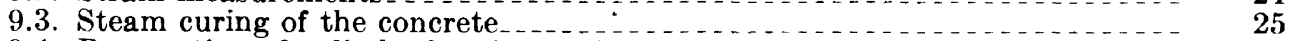

9.4. Preparation of cylinder bearing surfaces $\ldots \ldots \ldots$

9.5. Rate of loading for compressive strength test $\ldots \ldots \ldots$

9.6. Creep test $\ldots \ldots \ldots \ldots \ldots$

9.6.1. Creep test frame

9.6.2. Loading procedure

9.6.3. Effect of eccentricity of loading on creep measurements $\ldots$

9.6.4. Effect of loading time on creep measurements $\ldots$

9.7. Peculiarities of an anomalous lightweight aggregate $\ldots \ldots$ 



\title{
Creep and Drying Shrinkage of Lightweight and Normal-Weight Concretes
}

\author{
T. W. Reichard
}

\begin{abstract}
A description is given of a series of tests and test results from an investigation of the mechanical properties of structural-grade lightweight and normal-weight concretes. The major part of the work was planned to obtain comparative values of compressive creep, drying shrinkage, strengths, and moduli of elasticity for concretes made with 24 lightweight and 5 natural, normal-weight aggregates and having the same nominal strength at the time the specimens were placed under load.

Data are presented from a total of 76 different concretes which show that there is a wide range in values of the mechanical properties of concretes of the same nominal compressive strength. It is shown that relatively high values of creep and drying shrinkage are not always associated with lightweight concretes and that, in fact, some lightweight concretes have practically the same mechanical properties as some normal-weight concretes.

In addition to environmental conditions, the two major factors affecting the creep of concrete appear to be the ratio of the applied stress to the strength at the time of loading, and the aggregate used. Curing conditions, type of cement, mix proportions, and several other variables appear to affect the creep chiefly insofar as they affect the stress-strength ratio.

It was observed that the creep at the age of two years can be estimated from the 90-day results with reasonable accuracy.
\end{abstract}

\section{Introduction}

The trend toward lighter weight in structures coupled with the increasing scarcity, in some parts of the country, of good-quality natural concrete aggregates has led to the rapid increase in use of manufactured lightweight aggregates. Because structural-grade concretes can be readily made with many of these aggregates, large amounts are being used in concrete construction. Information on long-term length changes is needed because the stresses normally used in concrete construction are of such magnitude that proper allowances have to be made for loss of prestress and increase in deflection.

Some research on creep and drying shrinkage of lightweight aggregate concretes has been reported in the literature $[1,2,3$, and 4$] .{ }^{1}$ However, it was felt that a large group of the lightweight and normal-weight aggregates, representative of those being used in the construction industry, should be included in a single comprehensive investigation. The mix design, cure, and loading of the concretes should follow the general practice of the precast concrete industry. Although the major part of the data developed through this investigation will be of primary interest to the prestressed concrete industry, much of the data can be utilized by others interested in concrete construction.

The investigation was primarily designed to develop information on various mechanical properties of concretes having the same nominal compressive strength, but made with different aggregates.

This investigation was undertaken with the financial support of the Expanded Shale, Clay, and Slate Institute and with the cooperation of the producers of the aggregates.

\section{Scope of Investigation}

The work reported here is part of a continuing program concerning the long-term volume changes in concretes made from lightweight and normalweight aggregates. The creep, drying-shrinkage, and strength data presented are for concretes made with 24 expanded shale ${ }^{2}$ lightweight aggregates and 5 natural, normal-weight aggregates. These aggregates were from widely separated geographical locations and were to be representative samples of aggregates being used in the production of structural concretes.

For the major part of the work reported, hereafter called series A tests, the concretes were designed for a nominal 1-day compressive strength of 4000 psi using a 2 -in. slump and 5-percent air

\footnotetext{
1 Figures in brackets indicate the references at the end of this Monograph 2 In this report, "expanded shale aggregates" include shales, clays, an slates expanded in a horizontal rotating kiln.
}

content. The normal-weight aggregate concretes were designed using the 2 -in. slump, although for comparable workability with the lightweight concretes a 3 - or 4 -in. slump would have been preferable. Specimens of each concrete were placed under a long-term compressive creep load of 2000 psi when 1-day old. This creep load, which is a higher percentage of the strength at the time of loading than is recommended by a proposed ASTM creep method, was chosen as being representative of the practice in prestressing concrete members.

In effect, the series $A$ work was planned to give a comparison of the compressive creep and shrinkage characteristics of equal-strength concretes made with the various aggregates. Data are also presented from a series of supplemental tests, hereafter called series $B$, made to investigate variables other than the aggregates. 
The data reported here are from a total of 76 test batches of concrete. Of these, 63 batches were made with the 24 lightweight aggregates, 10 were made with the 5 normal-weight aggregates, and the other 3 batches were made with a combination of lightweight and normal-weight aggregates. Creep- and drying-shrinkage data, compressive and flexural strengths, modulus of elasticity, Poisson's ratio, and other data from these concretes are presented.

Details of the procedures and equipment used, as well as a description of the aggregates, are given in the appendix.

\section{Materials}

\subsection{Aggregates}

For the purposes of this investigation, all lightweight aggregates were designated by numbers and the normal-weight aggregates were designated by letters. All aggregates were to be representative samples of the production and were used in "as received" condition.

Sieve analysis and unit weights of each aggregate are given in table 1. All aggregates were received in bags holding 1 to $2 \mathrm{ft}^{3}$ of aggregate. Because of segregation of the fine aggregate while bagging, there were some differences in sieve analyses and unit weights from bag to bag. The values reported in table 1 are from samples taken from at least three bags.

The amount of aggregate passing the No. 200 sieve, as listed in table 1 , is not considered to be accurate for some of the lightweight aggregates. The test method used (ASTM C-136 and C-117) is thought to cause some of the larger particles to break down, especially when the more friable aggregates are being tested.

\subsubsection{Lightweight Aggregates}

All 24 lightweight aggregates used in this investigation were representative samples of the production at the time of shipment. However, due to changes in processing and/or raw materials the current production of a particular plant may be much different from what it was when the aggregate was received for this investigation. It should be noted that some of the aggregates were slightly heavier than the maximum dry-loose unit weight specified by AS'TM C-330-60'T.

The lightweight aggregates were shales, clays, or slates expanded in a horizontal rotary kiln. Although only about one third of these aggregates were considered to be "conted", nearly all contained some "coated" particles. When the aggregate particles are discharged from the rotary kiln, they are usually of a semirounded shape having a hard surface or shell and are commonly referred to as "conted particles." In some plants some or all of this product is crushed after burning. Aggregate particles produced by crushing after burning are commonly referred to as "crushed particles." The particle shape and surface texture of light- weight aggregate appear to affect the concretemaking ability in much the same way as they do for normal-weight aggregate. A fuller description of each aggregate is given in the appendix.

TABLE 1. Sieve analysis and unit weight of aggregates

\begin{tabular}{|c|c|c|c|c|c|c|c|c|c|c|c|c|c|}
\hline \multirow{2}{*}{$\begin{array}{c}\text { Aggregate } \\
\text { no. }\end{array}$} & \multirow{2}{*}{ Size } & \multirow{2}{*}{\begin{tabular}{|c} 
Unit \\
weight 1 \\
(I)ry \\
loose)
\end{tabular}} & \multicolumn{10}{|c|}{ Sieve analysis, percent passing } & \multirow{2}{*}{$\begin{array}{c}\text { Fine- } \\
\text { ness } \\
\text { modu- } \\
\text { lus }\end{array}$} \\
\hline & & & $8 / 4$ & $1 / 2$ & $8 / 8$ & 4 & 8 & 16 & 30 & 50 & 100 & 200 & \\
\hline 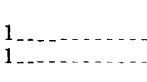 & $\begin{array}{l}\text { Fine } \\
\text { Int. }\end{array}$ & $\begin{array}{r}p c f \\
62.4 \\
44.2\end{array}$ & 100 & 100 & 100 & $\begin{array}{r}100 \\
22\end{array} \mid$ & $\begin{array}{l}89 \\
11\end{array}$ & $\begin{array}{r}62 \\
9\end{array}$ & 40 & \begin{tabular}{|c|}
27 \\
$-\cdots$
\end{tabular} & \begin{tabular}{|c|}
16 \\
$\cdots$
\end{tabular} & 10 & $\begin{array}{l}2.66 \\
5.58\end{array}$ \\
\hline $2 \ldots$ & $\begin{array}{l}\text { Fine } \\
\text { Int. }\end{array}$ & $\begin{array}{l}57.3 \\
46.2\end{array}$ & & 100 & $\mid \begin{array}{l}100 \\
100\end{array}$ & $\begin{array}{r}100 \\
80\end{array}$ & $\begin{array}{r}84 \\
8\end{array}$ & $\begin{array}{r}50 \\
2\end{array}$ & \begin{tabular}{r|}
31 \\
$-\cdots$
\end{tabular} & \begin{tabular}{|c|}
18 \\
$\cdots$
\end{tabular} & $\begin{array}{r}8 \\
--\end{array}$ & $\begin{array}{r}5 \\
-\end{array}$ & $\begin{array}{l}3.09 \\
5.09\end{array}$ \\
\hline 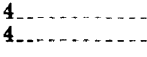 & $\begin{array}{l}\text { Fine } \\
\text { Int. }\end{array}$ & $\begin{array}{l}59.8 \\
47.2\end{array}$ & 100 & 100 & 99 & $\begin{array}{r}99 \\
9\end{array}$ & $\begin{array}{r}82 \\
3\end{array}$ & $\begin{array}{r}58 \\
0\end{array}$ & $\begin{array}{c}42 \\
---\end{array}$ & $\begin{array}{c}29 \\
-2\end{array}$ & $\mid \begin{array}{c}13 \\
--\end{array}$ & $\begin{array}{r}3 \\
--\end{array}$ & $\begin{array}{l}2.77 \\
5.89\end{array}$ \\
\hline 5 & $\begin{array}{l}\text { Fine } \\
\text { Int. }\end{array}$ & $\begin{array}{l}55.7 \\
45.3\end{array}$ & & 100 & $\begin{array}{r}100 \\
92\end{array}$ & $\begin{array}{l}98 \\
10\end{array}$ & $\begin{array}{r}81 \\
4\end{array}$ & $\begin{array}{r}50 \\
3\end{array}$ & 29 & 16 & 9 & 5 & $\begin{array}{l}3.18 \\
4.88\end{array}$ \\
\hline & $\begin{array}{l}\text { Fine } \\
\text { Int. }\end{array}$ & $\begin{array}{l}\text { 58. } \\
43.7\end{array}$ & 100 & 100 & 93 & $\begin{array}{r}100 \\
10\end{array}$ & $\begin{array}{r}90 \\
0\end{array}$ & $\begin{array}{r}64 \\
0\end{array}$ & 42 & 27 & $\begin{array}{r}18 \\
-\cdots\end{array}$ & $\begin{array}{r}9 \\
-\end{array}$ & $\begin{array}{l}2.59 \\
5.97\end{array}$ \\
\hline & $\begin{array}{l}\text { Fine } \\
\text { Int. }\end{array}$ & $\begin{array}{l}63.8 \\
55.2\end{array}$ & 100 & 100 & 94 & $\begin{array}{r}100 \\
9\end{array}$ & $\begin{array}{r}86 \\
2\end{array}$ & $\begin{array}{r}66 \\
0\end{array}$ & $\begin{array}{r}45 \\
-- \\
\end{array}$ & 29 & 19 & 14 & $\begin{array}{l}2.55 \\
5.95\end{array}$ \\
\hline & $\begin{array}{l}\text { Fine } \\
\text { Int. } \\
\text { Coarse }\end{array}$ & $\begin{array}{l}66.1 \\
55.0 \\
53.7\end{array}$ & 100 & $\begin{array}{l}100 \\
100\end{array}$ & 100 & $\left|\begin{array}{r}100 \\
94 \\
10\end{array}\right|$ & $\begin{array}{r}92 \\
8 \\
4\end{array}$ & \begin{tabular}{|r|}
65 \\
4 \\
0
\end{tabular} & 42 & 27 & \begin{tabular}{c}
17 \\
\hdashline \\
--
\end{tabular} & $\begin{array}{l}-. \\
--- \\
--\end{array}$ & $\begin{array}{l}2.57 \\
4.94 \\
5.86\end{array}$ \\
\hline $\begin{array}{l}10 \ldots \\
10 \ldots\end{array}$ & $\begin{array}{l}\text { Fine } \\
\text { Int. }\end{array}$ & $\begin{array}{l}70.2 \\
50.9\end{array}$ & 94 & . & 45 & $\begin{array}{r}100 \\
5\end{array}$ & $\begin{array}{r}92 \\
0\end{array}$ & $\begin{array}{r}59 \\
0\end{array}$ & $\begin{array}{r}37 \\
--\end{array}$ & 23 & 14 & $\begin{array}{r}7 \\
--\end{array}$ & $\begin{array}{l}2.75 \\
6.56\end{array}$ \\
\hline 1. & $\begin{array}{l}\text { Fine } \\
\text { Coarse. }\end{array}$ & $\begin{array}{l}62.0 \\
50.3\end{array}$ & 90 & 27 & -4 & $\begin{array}{r}100 \\
2\end{array}$ & $\begin{array}{r}84 \\
--\end{array}$ & $\begin{array}{r}51 \\
-\cdots\end{array}$ & 31 & 22 & 16 & 11 & $\begin{array}{l}2.96 \\
7.08\end{array}$ \\
\hline 4 & $\begin{array}{l}\text { Fine } \\
\text { Int. }\end{array}$ & $\begin{array}{l}45.0 \\
36.4\end{array}$ & 100 & 100 & 100 & $\begin{array}{r}100 \\
47\end{array}$ & $\begin{array}{r}96 \\
3\end{array}$ & $\begin{array}{r}67 \\
1\end{array}$ & $\begin{array}{r}39 \\
--\end{array}$ & 24 & 17 & 14 & $\begin{array}{l}2.57 \\
5.49\end{array}$ \\
\hline $5 \ldots$ & $\begin{array}{l}\text { Fine } \\
\text { Int. }\end{array}$ & $\begin{array}{l}60.4 \\
44.0\end{array}$ & 100 & 100 & 99 & $\begin{array}{r}100 \\
8\end{array}$ & $\begin{array}{r}85 \\
1\end{array}$ & $\begin{array}{r}57 \\
0\end{array}$ & 37 & 24 & 15 & $\begin{array}{r}8 \\
-.\end{array}$ & $\begin{array}{l}2.82 \\
5.92\end{array}$ \\
\hline$i_{2}$ & $\begin{array}{l}\text { Fine } \\
\text { Int. }\end{array}$ & $\begin{array}{l}61.5 \\
39.1\end{array}$ & 100 & 92 & 14 & $\begin{array}{r}100 \\
1\end{array} \mid$ & $\begin{array}{r}78 \\
0\end{array}$ & $\begin{array}{r}49 \\
0 \\
\end{array}$ & 27 & 12 & $\begin{array}{r}4 \\
--\end{array}$ & \begin{tabular}{r|}
1 \\
--
\end{tabular} & $\begin{array}{l}3.30 \\
6.85\end{array}$ \\
\hline$\cdots$ & $\begin{array}{l}\text { Fine } \\
\text { Int. }\end{array}$ & $\begin{array}{l}69.6 \\
47.4\end{array}$ & 100 & 87 & 49 & $\begin{array}{r}100 \\
12\end{array} \mid$ & $\begin{array}{r}81 \\
8\end{array}$ & $\begin{array}{r}52 \\
6 \\
\end{array}$ & 32 & 18 & 9 & $\begin{array}{c}4 \\
--\end{array}$ & $\begin{array}{l}3.08 \\
6.25\end{array}$ \\
\hline $\begin{array}{l}8 \\
8 \\
8\end{array}$ & $\begin{array}{l}\text { Fine } \\
\text { Int. }\end{array}$ & $\begin{array}{l}64.4 \\
38.6\end{array}$ & 100 & 100 & 100 & $\begin{array}{r}100 \\
34\end{array} \mid$ & $\begin{array}{r}89 \\
2\end{array}$ & $\begin{array}{r}65 \\
0\end{array}$ & 41 & $\begin{array}{c}19 \\
\cdots\end{array}$ & $\begin{array}{r}5 \\
-- \\
\end{array}$ & $\begin{array}{r}1 \\
-.\end{array}$ & $\begin{array}{l}2.81 \\
5.64\end{array}$ \\
\hline 20 & $\begin{array}{l}\text { Fine } \\
\text { Int. }\end{array}$ & $\begin{array}{r}58.6 \\
47.0\end{array}$ & 100 & 100 & 100 & $\left|\begin{array}{r}100 \\
71\end{array}\right|$ & $\begin{array}{r}93 \\
7\end{array}$ & \begin{tabular}{|r|}
60 \\
1 \\
\end{tabular} & 35 & 20 & 12 & 11 & $\begin{array}{l}2.80 \\
\text { 5. } 21\end{array}$ \\
\hline $1 \ldots$ & $\begin{array}{l}\text { Fine } \\
\text { Int. }\end{array}$ & $\begin{array}{l}44.2 \\
32.0\end{array}$ & -- & 100 & \begin{tabular}{|l|}
100 \\
100
\end{tabular} & $\left|\begin{array}{r}100 \\
25\end{array}\right|$ & $\begin{array}{r}73 \\
3\end{array}$ & \begin{tabular}{|r|}
48 \\
2 \\
\end{tabular} & 33 & 26 & 20 & 16 & $\begin{array}{l}\text { 3. } 00 \\
\text { 5. } 67\end{array}$ \\
\hline $22 \ldots$ & $\begin{array}{l}\text { Fine } \\
\text { Coarse }\end{array}$ & $\begin{array}{l}57.0 \\
38.3\end{array}$ & $9 \overline{4}$ & 48 & 25 & $\begin{array}{r}98 \\
6\end{array}$ & $\begin{array}{r}76 \\
4\end{array}$ & $\begin{array}{r}56 \\
3\end{array}$ & $\begin{array}{r}41 \\
-\ldots\end{array}$ & 30 & 19 & $\begin{array}{l}-. \\
-. \\
-1\end{array}$ & $\begin{array}{l}2.80 \\
6.46\end{array}$ \\
\hline $23 \ldots$ & $\begin{array}{l}\text { Fine } \\
\text { Int. }\end{array}$ & $\begin{array}{l}57.5 \\
50.7\end{array}$ & 100 & 96 & 64 & $\begin{array}{r}100 \\
3\end{array}$ & $\begin{array}{r}92 \\
1\end{array}$ & $\begin{array}{r}61 \\
0\end{array}$ & 35 & 17 & 7 & $\begin{array}{c}5 \\
--\end{array}$ & $\begin{array}{l}2.88 \\
6.32\end{array}$ \\
\hline & $\begin{array}{l}\text { Fine } \\
\text { Int. }\end{array}$ & $\begin{array}{l}73.7 \\
51.4\end{array}$ & 100 & 78 & 15 & $\begin{array}{r}100 \\
2\end{array}$ & $\begin{array}{r}95 \\
0\end{array}$ & $\begin{array}{r}59 \\
0\end{array}$ & 32 & 15 & 5 & \begin{tabular}{r|}
2 \\
--
\end{tabular} & $\begin{array}{l}2.94 \\
6.83\end{array}$ \\
\hline $\begin{array}{ll}25 \ldots \\
25 \ldots\end{array}$ & $\begin{array}{l}\text { Fine } \\
\text { Int. }\end{array}$ & $\begin{array}{l}68.2 \\
46.4\end{array}$ & 100 & 86 & 46 & $\begin{array}{r}100 \\
4\end{array}$ & $\begin{array}{r}86 \\
2\end{array}$ & $\mid \begin{array}{r}48 \\
0\end{array}$ & 30 & 20 & 14 & 11 & $\begin{array}{l}3.02 \\
6.48\end{array}$ \\
\hline & $\begin{array}{l}\text { Fine } \\
\text { Int. }\end{array}$ & $\begin{array}{c}54.0 \\
42.0\end{array}$ & 100 & 100 & 80 & $\begin{array}{r}100 \\
14\end{array}$ & $\begin{array}{r}85 \\
4\end{array}$ & $\begin{array}{r}59 \\
3\end{array}$ & 39 & 25 & 15 & 10 & $\begin{array}{l}2.77 \\
5.99\end{array}$ \\
\hline $27 \ldots$ & $\begin{array}{l}\text { Fine } \\
\text { Int. }\end{array}$ & $\begin{array}{l}70.5 \\
37.6\end{array}$ & 100 & 94 & 42 & $\begin{array}{r}100 \\
1\end{array}$ & $\begin{array}{r}89 \\
0\end{array}$ & $\begin{array}{r}64 \\
0\end{array}$ & 43 & 26 & 11 & 3 & $\begin{array}{l}2.67 \\
6.57\end{array}$ \\
\hline $28 \ldots$ & $\begin{array}{l}\text { Fine } \\
\text { Int. }\end{array}$ & $\begin{array}{l}61.9 \\
54.0\end{array}$ & 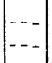 & $\ldots$ & 100 & $\left|\begin{array}{r}100 \\
65\end{array}\right|$ & $\begin{array}{r}100 \\
10\end{array}$ & $\mid \begin{array}{r}71 \\
1\end{array}$ & 45 & 26 & 12 & 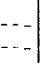 & $\begin{array}{l}2.47 \\
5.24\end{array}$ \\
\hline ) & $\begin{array}{l}\text { Fine } \\
\text { Int. }\end{array}$ & $\begin{array}{l}\text { 58. } 0 \\
43.6\end{array}$ & 98 & 58 & 24 & $\begin{array}{r}100 \\
1\end{array}$ & $\begin{array}{r}86 \\
0\end{array}$ & $\begin{array}{r}56 \\
0\end{array}$ & 35 & 23 & 15 & 11 & $\begin{array}{l}2.85 \\
6.77\end{array}$ \\
\hline & $\begin{array}{l}\text { Fine } \\
\text { Coarse }\end{array}$ & $\begin{array}{r}107.6 \\
96.1\end{array}$ & 100 & 52 & $\overline{2}$ & $\begin{array}{r}100 \\
1\end{array}$ & $\begin{array}{r}89 \\
0\end{array}$ & $\begin{array}{r}75 \\
0\end{array}$ & $\begin{array}{r}54 \\
-\end{array}$ & 17 & 8 & 2 & $\begin{array}{l}2.57 \\
6.97\end{array}$ \\
\hline$G G \& W M_{-}$ & Fines & 93.6 & & $\ldots$ & - & 97 & 88 & 76 & 59 & 21 & 5 & 2 & 2.54 \\
\hline GGA & Fines & 90.0 & & $\cdots$ & & 100 & 99 & 96 & 70 & 30 & 4 & 1 & 2.01 \\
\hline $\mathrm{RC}$ & Fines & & & $\ldots$ & & 100 & 79 & 52 & 32 & 14 & 4 & 2 & 3.19 \\
\hline$\Gamma \mathrm{R}$ & Fines & 98. & & & & 100 & 90 & 72 & 47 & 16 & 2 & 1 & 2. 73 \\
\hline
\end{tabular}

1 ASTM 330-60T. 


\subsubsection{Natural Dense Aggregates}

All five normal-weight aggregates in this investigation were commercially accepted for use in structural-grade concretes and were being used in large quantities for that purpose. All these aggregates were $3 / 4$-in. maximum size.

The aggregate designated $E$ was Elgin sand and gravel as used by Shideler [1] and [2]. The sand of aggregate $E$ had been screen separated and recombined before delivery. The coarse aggregate was supplied in three sizes. The aggregate designated $E D$ was also Elgin but was supplied and used as delivered to the trade in the Chicago area.

Aggregate R.G., known commercially as a granite, was a siliceous sand and gravel. This aggregate consisted of water-worn igneous stones of many varieties, none of which appeared to be granite.

Aggregate W.M. was a local sand and gravel, predominately quartzite. The gravel was separated into three sizes and recombined at batching.

Aggregate T.R. was a crushed trap rock from the northeastern United States. The fine fraction supplied with aggregate $T . R$. was a predominately quartzite sand.

The aggregate designated G.G. was a crushed light-gray granite and was supplied with a relatively fine quartzite sand. This sand was used in making one batch of concrete (G.G.A.). A second batch of concrete (G.G.) was made with this coarse aggregate and the local sand used with aggregate $W . M$.

\subsection{Cements}

All the cement of each type was received at one time and was specified to be from one silo. The type III cement which was used throughout the series A tests was stored in a sealed, dehumidified room in the export-style shipping bags. The type I cement which was used only in the series $B$ tests was stored in sealed steel drums.

Results of physical and chemical tests made by the NBS cement testing laboratory are shown in table 2. The results shown are for tests made about five months after receipt of the cements.

\subsection{Air-Entraining Agent}

A neutralized vinsol resin was used as an airentraining agent in all the concretes. This material, which was marketed as a 30 -percent solution, contained 26 percent of vinsol resin solids, 4 percent of $\mathrm{NaOH}$ solids and 70 percent of water. This solution was further diluted to a $7 \frac{1}{2}$ percent solution before using.

No other additive was used.

TABLE 2. Chemical and physical tests of cements 1

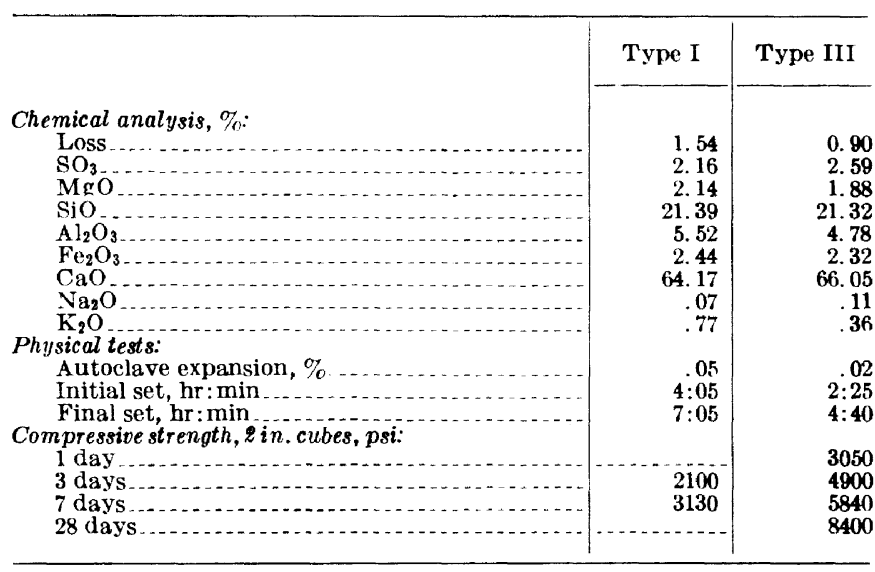

1 Federal Test Method Standard 158a.

\section{Concrete Mixes}

All concretes for series A tests were designed to have a nominal compressive strength of 4000 psi at 1 day using type III cement. To gain this high early strength without using excessive amounts of cement these concretes were cured for $12 \mathrm{hr}$ at atmospheric pressure in a steam chamber at $140^{\circ} \mathrm{F}$. This cure is roughly equivalent to the curing procedure used in the precast and prestressed concrete industry. All concretes were to be placed with a slump of 2 -in. and 5percent air content.

The concretes for series B tests were placed with the 2-in. slump and 5-percent air, but the nominal strength, cure, and type of cement varied from test to test. The specifications for each test within series B will be described later.

\subsection{Trial Mixes}

The producer of each lightweight aggregate had recommended a suitable mix for the series A concrete, but because of the variability in cements and other factors, at least three trial batches of concrete were made with each aggregate.
The proportion of fine to coarse aggregate was as recommended by the producer, but cement contents were varied so as to get a range in the compressive strength values. The amount of air-entraining agent was also adjusted during the trial batching.

The trial batches were mixed in a $3 / 4 \mathrm{ft}^{3}$ pan-type mixer. In mixing the concrete the light weight aggregates were mixed for $1 \mathrm{~min}$ with about $3 / 3$ of the estimated mix water and then allowed to stand for $10 \mathrm{~min}$; the air-entraining agent and the cement were then added and mixed for a minimum of $2 \mathrm{~min}$, additional water being added as was judged necessary for the required 2 -in. slump. The same mix cycle was used for the normal. weight aggregate concretes except that the 10 min waiting period was not used. Immediately after the mixer was emptied, samples of the concrete were taken for slump- and air-content measurements. The air content was determined by means of an air meter in accordance with ASTM C-173. Unit weight determinations were made by using the calibrated bowl of the air meter. 


\subsection{Test Concrete Mixes}

The amounts of cement, water, and air-entraining agent for the test concretes were selected after consideration of the trial batch results. Although these were used as a guide in the makeup of the test batches, the results of the latter were not uniformly the same, probably because of the differences in the two mixers used for trial batches and test batches and because of the absorption characteristics of the lightweight aggregates.
A $1 / 2-\mathrm{yd}^{3}$ turbine-type mixer was used for the $7-\mathrm{ft}^{3}$ test batches. Its mixing action was such that the amount of air-entraining agent necessary was considerably reduced from the estimate arrived at from the trial batches. The mix cycle and the sampling procedure were the same as used for the trial batches, except that the unit weight of the fresh concrete was determined by means of a $1 / 2-\mathrm{ft}^{3}$ measure.

Mix proportions for all concretes of series A are given in tables 3 and 7 .

\section{Preparation of Test Specimens}

\subsection{Description of Specimens}

Compressive strength, creep, and shrinkage specimens were 6 - by 12 -in. cylinders cast in machined cast-iron molds. Specimens for flexural strength and moisture expansion tests, which were made only for the series A concretes, were 3- by 4- by 16 -in. prisms cast in 4 -in. high steel molds. Gage points for the 10-in. Whittemore strain gage were cast in the creep, shrinkage, and moist ure expansion specimens. The creep and shrinkage specimens had three gage lines spaced uniformly around each cylinder. The moist ure expansion specimens had a gage line cast in each 4 - by 16 -in. face. A description of the gage points and the method of casting them in the specimen is given in the appendix (sec. 10.2).

\subsection{Casting of Specimens}

The concrete was consolidated in the molds by means of a laboratory-type internal vibrator. The concrete was placed in the mold in two equal layers. Each layer was vibrated by forcing the vibrator spud through the concrete once completely around the circumference of the specimen, or less if the concrete had subsided to a level plane. The spud was inserted through the top layer of concrete and slightly into the bottom layer when vibrating the top layer. Approximately $10 \mathrm{sec}$ of vibration were needed for each layer. No attempt was made to obtain a good cast surface on the specimens by external vibration.

The molds were slightly overfilled following the vibration, and the excess was struck off after the initial set of the concrete.

This vibration method of consolidating the concrete was used instead of rodding as it was thought to be more representative of the practice followed in placing concretes in precusting plants. A preliminary investigation regarding the merits of this method of vibration indicated that rodded cylinders (ASTM C-192) would yield slightly higher compressive strengths than the vibrated cylinders.

This method of ribration was designed to give satisfactory consolidation without overvibrating the concrete. Overvibration of the concrete would result in a loss of entrained air and possibly some segregation of the aggregate from the cement paste. The generally higher 1-day unit weights as compared with the plastic unit weights (table 3 ) is evidence, however, that some of the entrained air was lost even with the rather short vibration period used in this investigation.

\subsection{Curing of the Series A Concretes}

All series $A$ specimens were moved into an atmospheric-pressure, steam-curing chamber approximately $4 \mathrm{hr}$ after casting. The chamber was steam heated with a 2-stage electric steam generator. One stage of the generator had a relatively low capacity so that steam was being continuously introduced into the chamber throughout the curing cycle. The thermostatically controlled second stage of the generator was used to raise the temperature of the chamber up to the required $140{ }^{\circ} \mathrm{F}$ and to maintain it for the required $12 \mathrm{hr}$. After the steam-curing period the specimens were allowed to cool in the chamber for $2 \mathrm{hr}$ before demolding.

Immediately following removal from the molds, the specimens were weighed and the unit weight of the concrete recorded as the 1 day unit weight. All compressive strength specimens and half the flexure prisms were then stored in laboratory air controlled at $73.4^{\circ} \pm 4^{\circ} \mathrm{F}$. The other half of the flexural-test prisms were stored in the fog room controlled at $73.4^{\circ} \pm 3^{\circ} \mathrm{F}$.

The creep and shrinkage specimens were stored in a laboratory controlled at $72^{\circ} \pm 2^{\circ} \mathrm{F}$ and $50 \pm 3$ percent relative humidity.

\subsection{Preparation of Cylinder Bearing Surfaces}

All cylinders except the shrinkage specimens were prepared for loading by wet grinding the specimen ends in a surface grinder. A special jig was used to insure that the ends of the specimen would be ground perpendicular to the sides. This grinding was done shortly after removal from the molds. A description of the grinding method is given in the appendix.

This method of preparation of the bearing surfaces was used to a roid the use of special capping plates and capping materials for the creep specimens; grinding also eliminated the need of capping the compressive strength specimens. 
TABLE 3. Data for series A concretes

\begin{tabular}{|c|c|c|c|c|c|c|c|c|c|c|c|c|c|c|c|c|c|c|c|}
\hline \multirow{3}{*}{ Aggregate } & \multirow{3}{*}{ Batch } & \multicolumn{4}{|c|}{ Quantities per cu yd of concrete 1} & \multirow{3}{*}{$\begin{array}{l}\text { A ir } \\
\text { con- } \\
\text { tent }\end{array}$} & \multirow{3}{*}{ Slump } & \multirow{2}{*}{\multicolumn{5}{|c|}{ Unit weight }} & \multirow{2}{*}{\multicolumn{2}{|c|}{$\begin{array}{c}\text { Aggregate mois- } \\
\text { ture content } \\
\text { when batched } \\
\text { (by weight) }\end{array}$}} & \multirow{2}{*}{\multicolumn{5}{|c|}{$\begin{array}{l}\text { Compressive strength of } \\
\text { concrete, } f_{\mathrm{e}}^{3}\end{array}$}} \\
\hline & & \multirow[b]{2}{*}{ Water 2} & \multirow[b]{2}{*}{ Cement } & \multicolumn{2}{|c|}{$\begin{array}{l}\text { Aggregate } \\
\text { (oven dry) }\end{array}$} & & & & & & & & & & & & & & \\
\hline & & & & Fine & Coarse & & & Plastic & 1 day & $\begin{array}{c}\text { 28-day } \\
\text { test }\end{array}$ & $\begin{array}{c}1 \text { year } \\
\text { air } \\
\text { dried }\end{array}$ & $\begin{array}{c}\text { Oven } \\
\text { dry }\end{array}$ & Fines & Coarse & 1 day & 7 day & $\begin{array}{c}28 \\
\text { day }\end{array}$ & $\begin{array}{c}90 \\
\text { day }\end{array}$ & $\begin{array}{c}1 \\
\text { year }\end{array}$ \\
\hline 1. & $\begin{array}{l}\mathrm{A} \\
\mathrm{B} \\
\mathrm{C}\end{array}$ & $\begin{array}{c}l b \\
469 \\
462 \\
523\end{array}$ & $\begin{array}{r}l b \\
636 \\
655 \\
654\end{array}$ & $\begin{array}{c}l b \\
1040 \\
1051 \\
1046\end{array}$ & $\begin{array}{r}l b \\
701 \\
708 \\
708\end{array}$ & $\begin{array}{r}\% \\
4.0 \\
5.3 \\
5.0\end{array}$ & $\begin{array}{l}\text { in } \\
3.5 \\
3.3 \\
2.5\end{array}$ & $\begin{array}{c}p c f \\
104.8 \\
105.2 \\
105.6\end{array}$ & $\begin{array}{c}p c f \\
105.4 \\
106.6 \\
108.6\end{array}$ & $\begin{array}{c}p c f \\
101.4 \\
101.5 \\
102.9\end{array}$ & \begin{tabular}{|c|}
$p c f$ \\
99.0 \\
99.1 \\
100.4 \\
\end{tabular} & $\begin{array}{l}p c f \\
95.0 \\
95.0\end{array}$ & $\begin{array}{l}\% \\
0.1 \\
0 \\
0\end{array}$ & $\begin{array}{l}\% \\
0 \\
0 \\
0\end{array}$ & \begin{tabular}{|r|}
$p s i$ \\
3440 \\
3670 \\
$\mathbf{4 4 7 0}$ \\
\end{tabular} & $\begin{array}{r}p s i \\
4250 \\
4160 \\
5550\end{array}$ & $\begin{array}{r}p s i \\
4350 \\
4910 \\
6660\end{array}$ & $\begin{array}{r}p s i \\
4580 \\
5040 \\
7150\end{array}$ & $\begin{array}{r}p s i \\
4610 \\
4780 \\
6460\end{array}$ \\
\hline 2. & $\stackrel{\mathrm{A}}{\mathrm{B}}$ & $\begin{array}{l}482 \\
440\end{array}$ & $\begin{array}{l}561 \\
575\end{array}$ & $\begin{array}{r}1006 \\
969\end{array}$ & $\begin{array}{l}722 \\
698\end{array}$ & $\begin{array}{l}3.0 \\
5.0\end{array}$ & $\begin{array}{l}1.0 \\
1.8\end{array}$ & $\begin{array}{r}100.9 \\
95.4\end{array}$ & $\begin{array}{r}102.6 \\
99.3\end{array}$ & $\begin{array}{l}97.1 \\
94.6\end{array}$ & $\begin{array}{l}93.3 \\
90.2\end{array}$ & $\begin{array}{l}89.0 \\
88.0\end{array}$ & $\begin{array}{r}2.3 \\
.5\end{array}$ & $\begin{array}{r}1.8 \\
.5\end{array}$ & $\begin{array}{l}3800 \\
3790\end{array}$ & $\begin{array}{l}4970 \\
4870\end{array}$ & $\begin{array}{l}5950 \\
6130\end{array}$ & $\begin{array}{l}6120 \\
5900\end{array}$ & $\begin{array}{l}6020 \\
5310\end{array}$ \\
\hline 4. & A & $\begin{array}{l}523 \\
495\end{array}$ & $\begin{array}{l}605 \\
644\end{array}$ & $\begin{array}{l}945 \\
885\end{array}$ & $\begin{array}{l}642 \\
686\end{array}$ & $\begin{array}{l}4.0 \\
4.3\end{array}$ & $\begin{array}{l}1.5 \\
3.0\end{array}$ & $\begin{array}{r}100.6 \\
99.2\end{array}$ & \begin{tabular}{|l|}
100.6 \\
100.6
\end{tabular} & $\begin{array}{l}96.5 \\
95.7\end{array}$ & $\begin{array}{l}93.2 \\
92.5\end{array}$ & 88.9 & $\begin{array}{l}14.2 \\
14.7\end{array}$ & $\begin{array}{l}2 \\
1.7\end{array}$ & $\begin{array}{l}4130 \\
3940\end{array}$ & $\begin{array}{l}5310 \\
4840\end{array}$ & $\begin{array}{l}5690 \\
5460\end{array}$ & $\begin{array}{l}5600 \\
5500\end{array}$ & $\begin{array}{l}5720 \\
5210\end{array}$ \\
\hline 5 & A & $\begin{array}{l}635 \\
600\end{array}$ & $\begin{array}{l}627 \\
630\end{array}$ & $\begin{array}{l}878 \\
941\end{array}$ & $\begin{array}{l}631 \\
622\end{array}$ & $\begin{array}{l}3.5 \\
4.5\end{array}$ & $\begin{array}{l}2.3 \\
3.0\end{array}$ & $\begin{array}{l}101.8 \\
100.6\end{array}$ & $\begin{array}{l}102.9 \\
103.5\end{array}$ & $\begin{array}{l}96.1 \\
96.4\end{array}$ & $\begin{array}{l}92.7 \\
92.7\end{array}$ & $\begin{array}{l}86.0 \\
86.0\end{array}$ & $\begin{array}{l}6.6 \\
2.9\end{array}$ & $\begin{array}{l}1.3 \\
1.5\end{array}$ & $\begin{array}{l}4070 \\
4230\end{array}$ & $\begin{array}{l}5060 \\
4910\end{array}$ & $\begin{array}{l}5580 \\
5620\end{array}$ & $\begin{array}{l}5990 \\
6080\end{array}$ & $\begin{array}{l}5760 \\
6050\end{array}$ \\
\hline $6 \ldots$ & $\begin{array}{l}\mathrm{A} \\
\mathrm{B} \\
\mathrm{C}\end{array}$ & $\begin{array}{l}508 \\
434 \\
511\end{array}$ & $\begin{array}{l}583 \\
589 \\
610\end{array}$ & $\begin{array}{r}902 \\
1015 \\
899\end{array}$ & $\begin{array}{l}707 \\
677 \\
705\end{array}$ & $\begin{array}{l}5.5 \\
4.0 \\
4.8\end{array}$ & $\begin{array}{l}3.0 \\
1.5 \\
2.8\end{array}$ & $\begin{array}{r}98.1 \\
100.8 \\
99.3\end{array}$ & $\begin{array}{l}100.2 \\
100.5 \\
100.8\end{array}$ & $\begin{array}{l}94.1 \\
96.7 \\
95.1\end{array}$ & $\begin{array}{r}90.6 \\
992.4\end{array}$ & $\begin{array}{l}87.9 \\
89.3\end{array}$ & $\begin{array}{r}10.5 \\
10.6\end{array}$ & $\begin{array}{l}.1 \\
.1 \\
.1\end{array}$ & $\begin{array}{l}3350 \\
4720 \\
3730\end{array}$ & $\left|\begin{array}{l}4030 \\
6020 \\
4910\end{array}\right|$ & $\begin{array}{l}5240 \\
6580 \\
5660\end{array}$ & $\begin{array}{l}5350 \\
6490 \\
5670\end{array}$ & $\begin{array}{c}5120 \\
-5720\end{array}$ \\
\hline 8. & $\begin{array}{l}\mathrm{A} \\
\mathrm{B}\end{array}$ & $\begin{array}{l}477 \\
484\end{array}$ & $\begin{array}{l}629 \\
627\end{array}$ & $\begin{array}{l}1032 \\
1032\end{array}$ & $\begin{array}{l}712 \\
758\end{array}$ & $\begin{array}{l}5.5 \\
5.5\end{array}$ & $\begin{array}{l}3.0 \\
2.5\end{array}$ & $\begin{array}{l}104.2 \\
105.8\end{array}$ & $\begin{array}{l}105.6 \\
107.8\end{array}$ & $\begin{array}{l}100.4 \\
103.2\end{array}$ & $\begin{array}{l}97.8 \\
99.9\end{array}$ & $\begin{array}{l}93.9 \\
96.3\end{array}$ & $\begin{array}{l}2.7 \\
2.7\end{array}$ & $\begin{array}{l}6.8 \\
6.8\end{array}$ & $\begin{array}{l}4080 \\
4710\end{array}$ & $\begin{array}{l}4880 \\
5780\end{array}$ & $\begin{array}{l}5890 \\
6480\end{array}$ & $\begin{array}{l}5980 \\
6760\end{array}$ & $\begin{array}{l}5860 \\
6490\end{array}$ \\
\hline & $\begin{array}{l}\mathrm{A} \\
\mathrm{B}\end{array}$ & $\begin{array}{l}541 \\
516\end{array}$ & $\begin{array}{l}590 \\
600\end{array}$ & $\begin{array}{l}1162 \\
1177\end{array}$ & $\begin{array}{l}752 \\
768\end{array}$ & $\begin{array}{l}3.3 \\
3.5\end{array}$ & $\begin{array}{l}3.3 \\
2.8\end{array}$ & $\begin{array}{l}112.0 \\
111.6\end{array}$ & \begin{tabular}{|l|}
112.8 \\
113.3
\end{tabular} & $\begin{array}{l}106.2 \\
106.8\end{array}$ & $\begin{array}{l}103.6 \\
103.4\end{array}$ & 99.6 & $\begin{array}{l}3.5 \\
2.6\end{array}$ & $\begin{array}{l}8.0 \\
7.3\end{array}$ & $\begin{array}{l}4310 \\
4450\end{array}$ & $\begin{array}{l}5380 \\
5510\end{array}$ & $\begin{array}{l}6470 \\
6700\end{array}$ & $\begin{array}{l}6880 \\
7060\end{array}$ & $\begin{array}{l}6490 \\
6660\end{array}$ \\
\hline 10 & $\begin{array}{l}\mathrm{A} \\
\mathrm{B} \\
\mathrm{C}\end{array}$ & $\begin{array}{l}445 \\
468 \\
451\end{array}$ & $\begin{array}{l}562 \\
597 \\
524\end{array}$ & $\begin{array}{l}1074 \\
1127 \\
1076\end{array}$ & $\begin{array}{l}713 \\
694 \\
716\end{array}$ & $\begin{array}{l}4.0 \\
3.5 \\
3.5\end{array}$ & $\begin{array}{l}2.5 \\
2.5 \\
3.0\end{array}$ & $\begin{array}{l}101.4 \\
105.6 \\
101.0\end{array}$ & \begin{tabular}{|l|}
103.4 \\
107.0 \\
102.4
\end{tabular} & $\begin{array}{r}98.0 \\
100.7 \\
96.1\end{array}$ & \begin{tabular}{l|}
95.1 \\
98.8 \\
94.8
\end{tabular} & $\begin{array}{l}92.3 \\
95.2\end{array}$ & $\begin{array}{l}0 \\
0 \\
.3\end{array}$ & $\begin{array}{l}0 \\
0 \\
0\end{array}$ & $\begin{array}{l}3620 \\
4410 \\
3160\end{array}$ & $\begin{array}{l}4350 \\
5220 \\
4120\end{array}$ & $\begin{array}{l}5170 \\
5920 \\
4580\end{array}$ & $\begin{array}{l}5600 \\
6160 \\
5040\end{array}$ & $\begin{array}{l}5560 \\
6160 \\
4960\end{array}$ \\
\hline 14 & $\begin{array}{l}\mathrm{A} \\
\mathrm{B}\end{array}$ & $\begin{array}{l}596 \\
571\end{array}$ & $\begin{array}{l}668 \\
657\end{array}$ & $\begin{array}{l}786 \\
840\end{array}$ & $\begin{array}{l}548 \\
555\end{array}$ & $\begin{array}{l}4.5 \\
5.4\end{array}$ & $\begin{array}{l}2.8 \\
1.8\end{array}$ & \begin{tabular}{l|l|}
94.6 \\
94.8
\end{tabular} & $\begin{array}{l}96.3 \\
97.0\end{array}$ & $\begin{array}{l}89.7 \\
90.4\end{array}$ & $\begin{array}{l}83.7 \\
86.0\end{array}$ & $\begin{array}{l}77.2 \\
79.3\end{array}$ & $\begin{array}{l}8.0 \\
5.6\end{array}$ & $\begin{array}{r}10.0 \\
9.0\end{array}$ & \begin{tabular}{|l|}
4900 \\
5770
\end{tabular} & $\begin{array}{l}5830 \\
6330\end{array}$ & $\begin{array}{l}6140 \\
6710\end{array}$ & $\begin{array}{l}5970 \\
6790\end{array}$ & $\begin{array}{l}5740 \\
6480\end{array}$ \\
\hline $15 \ldots$ & $\mathrm{A}$ & $\begin{array}{l}441 \\
439\end{array}$ & $\begin{array}{l}562 \\
593\end{array}$ & $\begin{array}{l}1029 \\
1043\end{array}$ & $\begin{array}{l}637 \\
646\end{array}$ & $\begin{array}{l}6.5 \\
3.8\end{array}$ & $\begin{array}{l}3.5 \\
2.3\end{array}$ & $\begin{array}{l}96.6 \\
99.0\end{array}$ & $\begin{array}{r}98.8 \\
100.7\end{array}$ & $\begin{array}{l}93.3 \\
96.4\end{array}$ & $\begin{array}{l}90.6 \\
92.9\end{array}$ & $\begin{array}{l}88.3 \\
89.8\end{array}$ & $\begin{array}{l}0 \\
0\end{array}$ & $\begin{array}{l}0 \\
0\end{array}$ & $\begin{array}{l}3520 \\
4400\end{array}$ & $\begin{array}{l}4180 \\
5340\end{array}$ & $\begin{array}{l}5460 \\
6640\end{array}$ & $\begin{array}{l}5520 \\
6770\end{array}$ & $\begin{array}{l}5530 \\
6230\end{array}$ \\
\hline $166_{--}$ & $\begin{array}{l}\mathrm{A} \\
\mathrm{B}\end{array}$ & $\begin{array}{l}369 \\
373\end{array}$ & $\begin{array}{l}544 \\
588\end{array}$ & $\begin{array}{l}1118 \\
1096\end{array}$ & $\begin{array}{l}579 \\
565\end{array}$ & $\begin{array}{l}5.0 \\
4.5\end{array}$ & $\begin{array}{l}1.8 \\
2.3\end{array}$ & $\begin{array}{l}96.2 \\
96.3\end{array}$ & $\begin{array}{l}96.6 \\
97.2\end{array}$ & $\begin{array}{l}93.8 \\
93.8\end{array}$ & $\begin{array}{l}91.7 \\
92.3\end{array}$ & 88.6 & $\begin{array}{l}0 \\
0\end{array}$ & $\begin{array}{l}0 \\
0\end{array}$ & $\begin{array}{l}3850 \\
4240\end{array}$ & $\begin{array}{l}4600 \\
4370\end{array}$ & \begin{tabular}{|l|}
5260 \\
4630
\end{tabular} & $\begin{array}{l}4900 \\
5240\end{array}$ & $\begin{array}{l}5040 \\
4980\end{array}$ \\
\hline 17. & $\begin{array}{l}\mathrm{A} \\
\mathrm{B}\end{array}$ & $\begin{array}{l}465 \\
448\end{array}$ & $\begin{array}{l}585 \\
591\end{array}$ & $\begin{array}{l}1250 \\
1240\end{array}$ & $\begin{array}{l}621 \\
618\end{array}$ & $\begin{array}{l}\text { 4. } 0 \\
\text { 3. } 8\end{array}$ & $\begin{array}{l}3.0 \\
2.5\end{array}$ & $\begin{array}{l}106.6 \\
105.9\end{array}$ & $\begin{array}{l}108.2 \\
107.3\end{array}$ & $\begin{array}{l}102.4 \\
102.4\end{array}$ & $\begin{array}{r}99.2 \\
101.1\end{array}$ & 97.4 & $\begin{array}{l}2.2 \\
2.2\end{array}$ & $\begin{array}{l}1.5 \\
1.5\end{array}$ & $\begin{array}{l}4190 \\
4590\end{array}$ & $\begin{array}{l}\mathbf{5 4 9 0} \\
\mathbf{5 4 8 0}\end{array}$ & $\begin{array}{l}6270 \\
6100\end{array}$ & $\begin{array}{l}6070 \\
6570\end{array}$ & $\begin{array}{l}6120 \\
6370\end{array}$ \\
\hline $18 .$. & $\begin{array}{l}\mathrm{A} \\
\mathrm{B}\end{array}$ & $\begin{array}{l}383 \\
369\end{array}$ & $\begin{array}{l}591 \\
580\end{array}$ & $\begin{array}{l}929 \\
912\end{array}$ & $\begin{array}{l}623 \\
612\end{array}$ & $\begin{array}{l}6.0 \\
5.0\end{array}$ & $\begin{array}{l}2.0 \\
2.5\end{array}$ & $\begin{array}{l}91.6 \\
90.0\end{array}$ & $\begin{array}{l}93.5 \\
91.4\end{array}$ & $\begin{array}{l}89.4 \\
89.3\end{array}$ & $\begin{array}{l}87.1 \\
86.9\end{array}$ & 83.6 & .1 & $\begin{array}{l}0 \\
0\end{array}$ & $\begin{array}{l}4420 \\
3810\end{array}$ & $\begin{array}{l}4940 \\
4560\end{array}$ & $\begin{array}{l}5720 \\
5560\end{array}$ & $\begin{array}{l}5290 \\
5410\end{array}$ & $\begin{array}{l}5250 \\
5360\end{array}$ \\
\hline 20 & $\begin{array}{l}\mathrm{A} \\
\mathrm{B}\end{array}$ & $\begin{array}{l}524 \\
516\end{array}$ & $\begin{array}{l}644 \\
650\end{array}$ & $\begin{array}{l}1024 \\
1016\end{array}$ & $\begin{array}{l}554 \\
550\end{array}$ & $\begin{array}{l}4.5 \\
4.0\end{array}$ & $\begin{array}{l}2.3 \\
2.0\end{array}$ & $\begin{array}{l}101.1 \\
100.4\end{array}$ & $\begin{array}{l}101.7 \\
101.3\end{array}$ & \begin{tabular}{l|}
96.1 \\
95.9
\end{tabular} & $\begin{array}{l}92.4 \\
93.8\end{array}$ & 89.1 & .1 & $\begin{array}{l}.4 \\
.4\end{array}$ & $\begin{array}{l}4260 \\
4590\end{array}$ & $\begin{array}{l}5580 \\
5690 \\
\end{array}$ & $\begin{array}{l}6720 \\
6340\end{array}$ & $\begin{array}{l}6610 \\
7200\end{array}$ & $\begin{array}{l}6340 \\
6730\end{array}$ \\
\hline $21 \ldots$ & $\begin{array}{l}\mathrm{A} \\
\mathrm{B}\end{array}$ & $\begin{array}{l}567 \\
572\end{array}$ & $\begin{array}{l}684 \\
672\end{array}$ & $\begin{array}{l}776 \\
784\end{array}$ & $\begin{array}{l}479 \\
484\end{array}$ & $\begin{array}{l}3.5 \\
4.5\end{array}$ & $\begin{array}{l}2.5 \\
2.5\end{array}$ & $\begin{array}{l}90.6 \\
89.4\end{array}$ & $\begin{array}{l}92.9 \\
93.0\end{array}$ & $\begin{array}{l}86.1 \\
86.4\end{array}$ & $\begin{array}{l}81.5 \\
82.7\end{array}$ & $\begin{array}{l}78.0 \\
78.0\end{array}$ & $\begin{array}{l}0 \\
0\end{array}$ & $\begin{array}{l}0 \\
0\end{array}$ & $\begin{array}{l}4000 \\
3760\end{array}$ & $\begin{array}{l}4370 \\
4510\end{array}$ & $\begin{array}{l}\mathbf{4 8 0 0} \\
\mathbf{4 6 8 0}\end{array}$ & $\begin{array}{l}4910 \\
5020\end{array}$ & $\begin{array}{l}4380 \\
4570\end{array}$ \\
\hline $23 \ldots$ & $\begin{array}{l}\mathrm{A} \\
\mathrm{B} \\
\mathrm{C}\end{array}$ & $\begin{array}{l}471 \\
473 \\
415\end{array}$ & $\begin{array}{l}615 \\
605 \\
545\end{array}$ & $\begin{array}{l}1026 \\
1024 \\
1106\end{array}$ & $\begin{array}{l}771 \\
771 \\
750\end{array}$ & $\begin{array}{l}5.0 \\
4.5 \\
5.5\end{array}$ & $\begin{array}{l}1.8 \\
2.3 \\
2.5\end{array}$ & $\begin{array}{r}104.6 \\
105.4 \\
99.8\end{array}$ & $\begin{array}{l}106.7 \\
106.5 \\
104.1\end{array}$ & $\begin{array}{r}101.5 \\
102.2 \\
98.7\end{array}$ & $\begin{array}{l}98.9 \\
99.0 \\
96.3\end{array}$ & $\begin{array}{l}96.0 \\
95.4 \\
93.0\end{array}$ & $\begin{array}{r}7.0 \\
6.4 \\
.4\end{array}$ & $\begin{array}{r}2.0 \\
1.7 \\
.3\end{array}$ & $\begin{array}{l}5170 \\
4560 \\
4530\end{array}$ & $\begin{array}{l}6000 \\
5450 \\
5510\end{array}$ & $\begin{array}{l}7460 \\
6370 \\
6630\end{array}$ & & $\begin{array}{l}7180 \\
6770 \\
6080\end{array}$ \\
\hline 24 & $\begin{array}{l}\text { A } \\
\text { B }\end{array}$ & $\begin{array}{l}370 \\
357\end{array}$ & $\begin{array}{l}570 \\
555\end{array}$ & $\begin{array}{l}1250 \\
1288\end{array}$ & $\begin{array}{l}714 \\
733\end{array}$ & $\begin{array}{l}7.0 \\
6.3\end{array}$ & $\begin{array}{l}3.3 \\
2.3\end{array}$ & \begin{tabular}{|l|}
104.7 \\
105.2
\end{tabular} & \begin{tabular}{|l|}
107.7 \\
108.5
\end{tabular} & $\begin{array}{l}103.5 \\
106.0\end{array}$ & $\begin{array}{l}101.0 \\
102.4\end{array}$ & $\begin{array}{l}99.1 \\
98.9\end{array}$ & .5 & $\begin{array}{l}0 \\
0\end{array}$ & $\begin{array}{l}4680 \\
5110\end{array}$ & $\begin{array}{l}5440 \\
6049\end{array}$ & $\begin{array}{l}6440 \\
6860\end{array}$ & $\begin{array}{l}6730 \\
7590\end{array}$ & \begin{tabular}{|l|}
6300 \\
6540
\end{tabular} \\
\hline $25 \ldots$ & $\begin{array}{l}\mathrm{A} \\
\mathrm{B}\end{array}$ & $\begin{array}{l}395 \\
410\end{array}$ & $\begin{array}{l}626 \\
613\end{array}$ & $\begin{array}{l}1137 \\
1115\end{array}$ & $\begin{array}{l}663 \\
650\end{array}$ & $\begin{array}{l}4.0 \\
3.5\end{array}$ & $\begin{array}{l}2.0 \\
3.0\end{array}$ & \begin{tabular}{|l|}
102.3 \\
102.0
\end{tabular} & $\begin{array}{l}104.5 \\
103.2\end{array}$ & $\begin{array}{l}101.3 \\
100.8\end{array}$ & $\begin{array}{l}98.7 \\
98.4\end{array}$ & 94.8 & $\begin{array}{l}0 \\
0\end{array}$ & $\begin{array}{l}0 \\
0\end{array}$ & $\begin{array}{l}4360 \\
4060\end{array}$ & $\begin{array}{l}5820 \\
5330\end{array}$ & $\begin{array}{l}7090 \\
6180\end{array}$ & $\begin{array}{l}6730 \\
6080\end{array}$ & $\begin{array}{l}6560 \\
5900\end{array}$ \\
\hline 26. & $\begin{array}{l}\text { A } \\
\text { B }\end{array}$ & $\begin{array}{l}431 \\
439\end{array}$ & $\begin{array}{l}740 \\
741\end{array}$ & $\begin{array}{l}832 \\
832\end{array}$ & $\begin{array}{l}64 \\
64\end{array}$ & $\begin{array}{l}5.0 \\
4.3\end{array}$ & $\begin{array}{l}3.0 \\
3.0\end{array}$ & $\begin{array}{l}97.0 \\
96.2\end{array}$ & $\begin{array}{l}98.1 \\
98.5\end{array}$ & $\begin{array}{l}93.1 \\
95.1\end{array}$ & $\begin{array}{l}90.2 \\
92.9\end{array}$ & 89.0 & $\begin{array}{l}.3 \\
.3\end{array}$ & $\begin{array}{l}.3 \\
.3\end{array}$ & $\begin{array}{l}4140 \\
4080\end{array}$ & $\begin{array}{l}4770 \\
5400\end{array}$ & $\begin{array}{l}5240 \\
5940\end{array}$ & $\begin{array}{l}5420 \\
5860\end{array}$ & $\begin{array}{l}4650 \\
5450\end{array}$ \\
\hline $27 .$. & A & $\begin{array}{l}401 \\
365\end{array}$ & $\begin{array}{l}604 \\
619\end{array}$ & $\begin{array}{l}1086 \\
1128\end{array}$ & $\begin{array}{l}521 \\
523\end{array}$ & $\begin{array}{l}7.3 \\
5.5\end{array}$ & $\begin{array}{l}2.5 \\
2.8\end{array}$ & $\begin{array}{l}95.3 \\
96.6\end{array}$ & $\begin{array}{l}96.8 \\
97.5\end{array}$ & $\begin{array}{l}92.2 \\
93.4\end{array}$ & $\begin{array}{l}90.4 \\
91.6\end{array}$ & 89.2 & $\begin{array}{l}3.2 \\
0\end{array}$ & $\begin{array}{l}0 \\
0\end{array}$ & $\begin{array}{l}3640 \\
3990\end{array}$ & $\begin{array}{l}4060 \\
4740\end{array}$ & $\begin{array}{l}4560 \\
5000\end{array}$ & $\begin{array}{l}4540 \\
5050\end{array}$ & $\begin{array}{l}4650 \\
5020\end{array}$ \\
\hline 30 & $\begin{array}{l}\mathrm{A} \\
\mathrm{B}\end{array}$ & $\begin{array}{l}505 \\
474\end{array}$ & $\begin{array}{l}585 \\
578\end{array}$ & $\begin{array}{l}969 \\
974\end{array}$ & $\begin{array}{l}648 \\
645\end{array}$ & $\begin{array}{l}5.0 \\
4.3\end{array}$ & 3. 0 & $\begin{array}{l}98.6 \\
98.6\end{array}$ & $\begin{array}{r}100.0 \\
99.1\end{array}$ & $\begin{array}{l}94.6 \\
94.2\end{array}$ & $\begin{array}{l}91.5 \\
91.3\end{array}$ & 88.6 & $\begin{array}{l}5.8 \\
5.0\end{array}$ & $\begin{array}{r}1.0 \\
.5\end{array}$ & $\begin{array}{l}3900 \\
3640\end{array}$ & $\begin{array}{l}4910 \\
4600\end{array}$ & $\begin{array}{l}5570 \\
5800\end{array}$ & $\begin{array}{l}6110 \\
5845\end{array}$ & $\begin{array}{l}5630 \\
5750\end{array}$ \\
\hline $\mathrm{E}_{-}$ & $\begin{array}{l}\mathrm{A} \\
\mathrm{B}\end{array}$ & $\begin{array}{l}261 \\
288\end{array}$ & $\begin{array}{l}456 \\
452\end{array}$ & $\begin{array}{l}1240 \\
1234\end{array}$ & $\begin{array}{l}2018 \\
2002\end{array}$ & $\begin{array}{l}4.0 \\
3.8\end{array}$ & $\begin{array}{l}1.5 \\
3.0\end{array}$ & $\begin{array}{l}146.0 \\
146.0\end{array}$ & $\begin{array}{l}147.6 \\
147.2\end{array}$ & $\begin{array}{l}146.6 \\
145.3\end{array}$ & $\begin{array}{r}143.9 \\
142.9\end{array}$ & 142.5 & $\begin{array}{l}0 \\
.2\end{array}$ & $\begin{array}{l}0 \\
0\end{array}$ & $\begin{array}{l}3870 \\
3680\end{array}$ & $\begin{array}{l}5409 \\
5350\end{array}$ & $\begin{array}{l}6490 \\
5880\end{array}$ & $\begin{array}{l}6390 \\
6030\end{array}$ & $\begin{array}{l}6500 \\
5720\end{array}$ \\
\hline EI). & & 304 & 462 & 1226 & 1958 & 5.0 & 3.0 & 144.6 & 146.5 & 143.4 & & & 0 & 0 & 3550 & 4640 & 5300 & & \\
\hline $\mathrm{GG}_{-}$ & & 266 & 476 & 1184 & 1860 & 6.2 & 2.5 & 139.2 & 140.2 & 139.1 & & & .4 & 0 & 4080 & 4840 & 5250 & & \\
\hline GGA & & 310 & 497 & 1133 & 1940 & 5.5 & 3.0 & 141.2 & 143.5 & 141.2 & & & 0 & 0 & 3930 & & 5780 & & \\
\hline $\mathrm{KG}_{-}$ & & 282 & 455 & 1225 & 2060 & 4.0 & 1.8 & 146.2 & 149.0 & 146.9 & & & 0 & 0 & 3260 & 4330 & 4840 & & \\
\hline $\mathrm{TR}$ & & 290 & 498 & 1304 & 1982 & 4.4 & 2.5 & 149.0 & 151.0 & 149.1 & & & 4 & 0 & 4470 & 5200 & 5790 & & \\
\hline WM. & & 244 & 478 & 1189 & 1955 & 5. 0 & 1.5 & 141.5 & 143.4 & 143.1 & & & 0 & ) & 4410 & 5280 & 5900 & & \\
\hline
\end{tabular}

1 Based on unit weight as demolded.

2 Includes moisture in aggregate.

${ }^{3}$ A verage of 2 specimens. 


\section{Test Procedure}

\subsection{Compressive Strength Test}

The compressive strength was determined for most of the concretes at the ages of $1,7,28,90$, and 365 days. $^{3}$ Two 6 - by 12 -in. cylinders were used as a sumple at each age.

The specimens, which were prepared for test by the grinding mentioned in sec. 5.4, were loaded until failure in a 300,000-lb capacity hydraulic testing machine. The failures in most specimens of the lightweight concretes were sudden and sometimes explosive. The type of failure in general was the usual double cone, but in some specimens vertical splitting occurred.

Stress-strain determinations were made on one of the specimens from each sample. Strain readings were made, by means of 6 -in. bonded wire strain gages, at convenient load increments without interrupting the continuous application of the load which was continued until failure of the specimen. The strain gages were bonded to the specimens with an epoxy resin cement except for the one-day test. A modified cyanoacrylate was used to bond the gages on the damp one-day specimens.

The secant modulus of elasticity at two stress levels was computed from the stress-strain data for each sample. These secunt values were determined for stress levels of 0.4 and 0.9 of the compressive strength.

The secant modulus differs theoretically from the chord modulus specified in ASTM C-469-61T, but is identical in value for the data developed in this investigation.

The rate of loading for the stress-strain specimens was about $22 \mathrm{psi} / \mathrm{sec}$. The other compressive strength specimens were loaded at $44 \mathrm{psi} / \mathrm{sec}$.

\subsection{Dynamic Modulus of Elasticity and Poisson's Ratio}

The dynamic modulus of elasticity $\left(E_{D}\right)$ and Poisson's ratio was determined on all compressive test specimens using the resonance method described in ASTM C-215-60. $E_{D}$ was calculated from the fundamental longitudinal frequency. Poisson's ratio was calculated from the fundamental longitudinal and torsional frequencies.

\subsection{Flexural Strength Test}

The modulus of rupture of the series A concretes was determined on the 3- by 4 - by 16 -in. prisms using ASTM Test Method $\dot{C}^{-78-57}$. Determinations were made at the ages of 1,28 , and

3 A limited supply of aggregate reduced the number of specimens cast from concretes ED, G.G.A., R.G., T.R., and II.M. for series A tests.
365 days. Two specimens were tested at the age of one day shortly after removal from the molds. Four specimens were tested at each of the other agres, with two being laboratory air-dried and the other pair being continuously moist cured.

The test height of the prism was 3 in. as the height at casting was 4 in. Two breaks were made on each specimen.

\subsection{Creep and Shrinkage Test}

All creep specimens representing series A concretes were placed under load when about $28 \mathrm{hr}$ old, but the series B concretes were loaded at other ages and will be described later. The loading frame was essentially the same as used by Shideler [1] except that the height was increased to allow for half-length dummy concrete specimens at the top and bottom of the set of three test specimens in each frame. The dummy cylinders were necessary in order to comply with a test method being prepared by ASTM Committee C-9.

Four specimens were used as a sample of each concrete. One specimen from each sample was loaded in each of two creep frames. Two cylinders were left unloaded to serve as shrinkage specimens. Thus, each creep frame contained test specimens representing three different concretes.

Considerable care was used in setting up the creep frame and in placing the specimens in the frame in order to reduce to a minimum the eccentricity in the load on the specimens. The load was applied by means of a hydraulic ram through a load cell. Eccentricity of loading was checked by observing the variation of the deformation in the three gage lines of the specimens at low loads. If the variation was observed to be greater than 10 percent, the load was immediately removed and specimens were rotated relative to each other until the variation was reduced. The load applied to the specimens was estimated to be within 1 percent of the required load. The loading frames, which were reloaded periodically, had a maximum observed drop-off in load, prior to reloading, of 5 percent. Details of the test frame and loading procedure are given in the appendix.

All deformations in the creep and shrinkage specimens were measured with the same Whittemore strain gage. The mild steel standard bar, used for temperature corrections of the strain gage readings, was stored on a stone slab in a box of 3/4-in. plywood. This procedure was used in order to reduce temperature variations in the standard bar caused by the forced air currents in the temperature controlled laboratory. All gage readings were estimated to $0.00002 \mathrm{in}$. which, for the 10 -in. gage length, was a strain of 0.0002 percent. 


\section{Results}

\subsection{Results-Series A Tests}

\subsubsection{Compressive Strength Test Results}

No special problems were encountered in obtaining the required compressive strength of concrete for the aggregates tested. However, with the limited experience acquired from each aggregate, some errors were made in obtaining the required slump and air content. No attempt was made to obtain identical concretes when additional batches were made from a given aggregate. In some instances, such batches were deliberately designed to be different.

The test data listed in tables 3 and 7 and shown in figure 1 are for the series $A$ concretes. The data presented in figure 1 for the lightweight concretes are averages for all the lightweight concretes, while the data for the normal-weight concretes are averages of the two batches made from aggregate $E$.

Probably the most significant aspect of the compressive-strength data is the slight loss of compressive strength in most of these steani-cured concretes after 90 days. This retrogression, which has been reported by others, is usually attributed to microscopic shrinkage cracks which occur with the air drying after the steam cure. However, it may be significant that the continuously moistcured lightweight concrete specimens showed no increase in flexural strength between 28 days and one year, whereas the air-dried normal-weight concrete specimens showed greater flexural strengths than the companion moist-cured specimens at all ages.
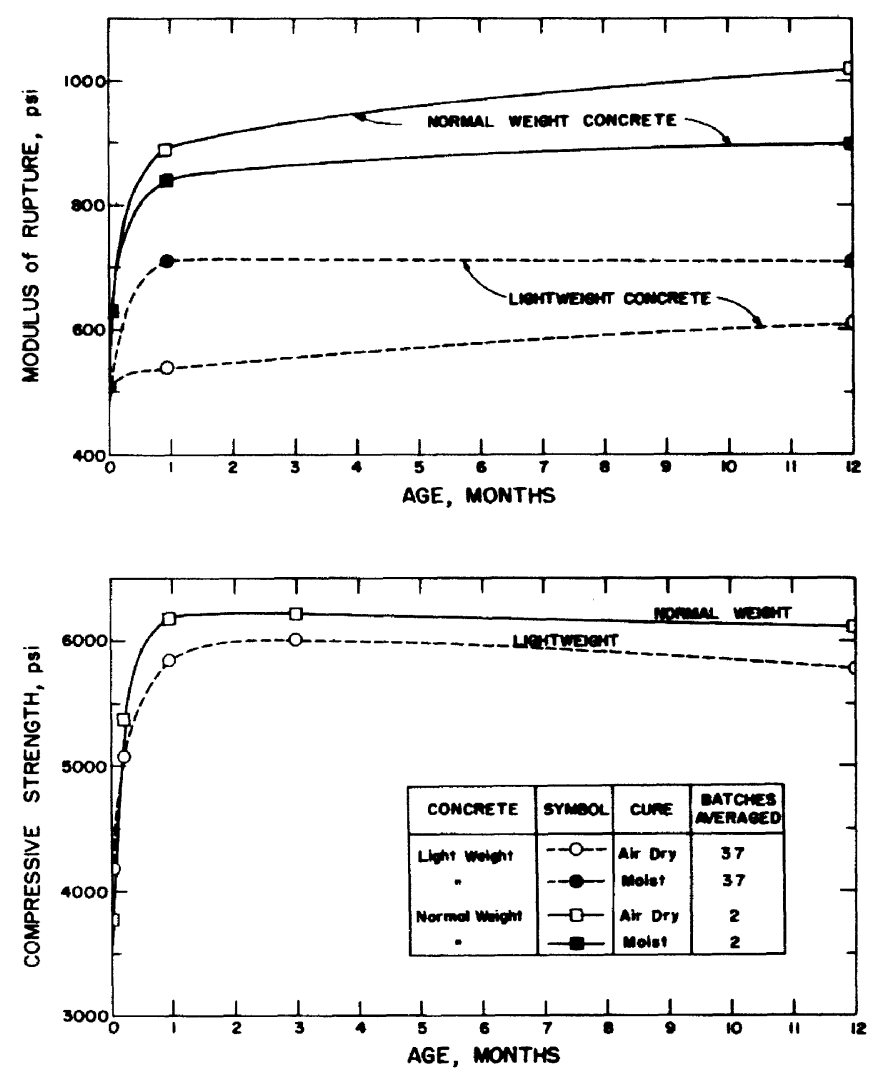

FiguRe 1. Average compressive- and flexural-strength test results for series A concretes at various ages.

All concretes were stean-cured for $12 \mathrm{hr}$ at $140^{\circ} \mathrm{F}$ and were made with type III cement.

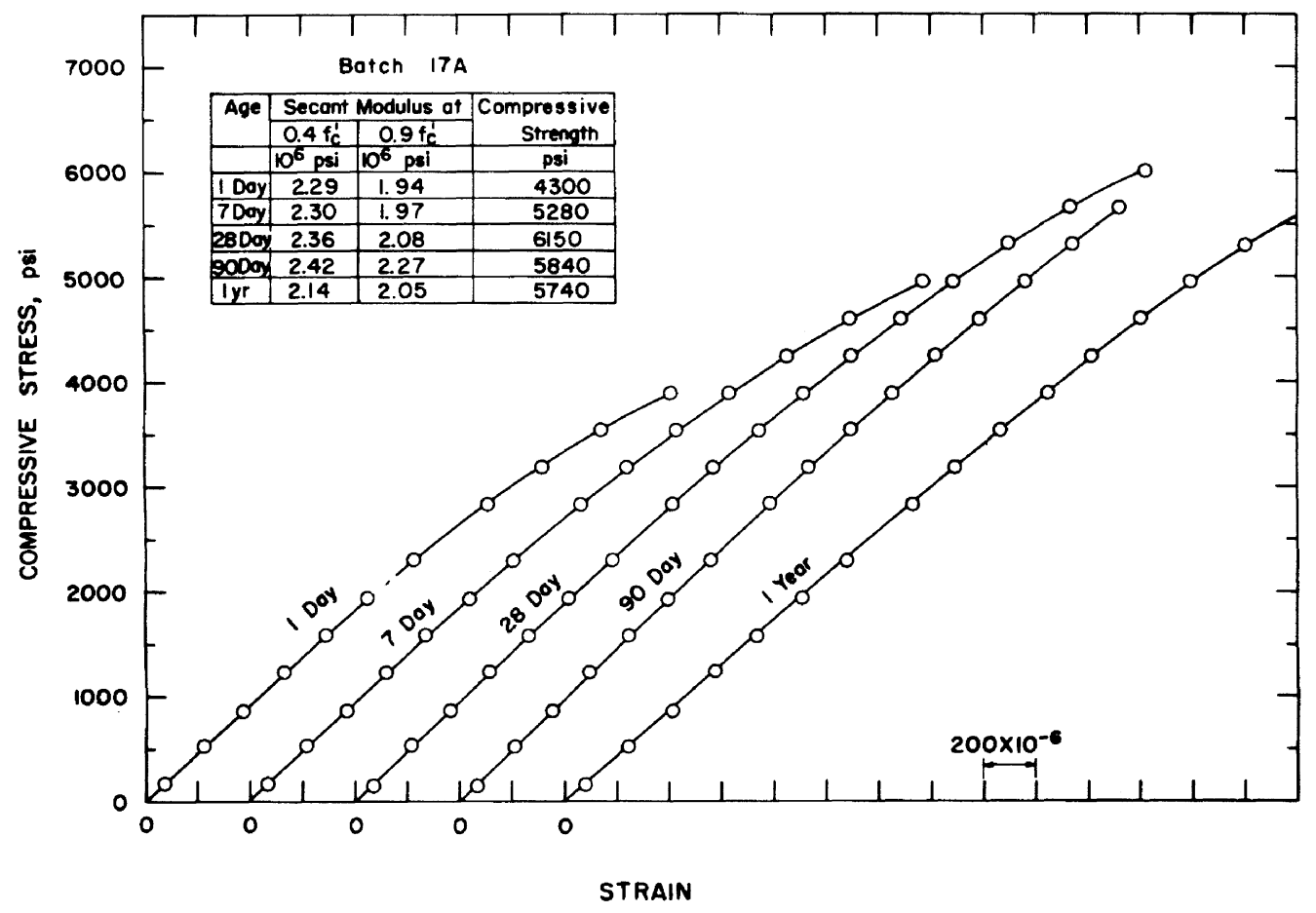

Figure 2. Typical stress-strain curves for a lightweight concrete. 


\subsubsection{Moduli of Elasticity and Poisson's Ratio}

A set of typical stress-strain curves determined on compressive strength specimens is shown in figure 2. From curves such as these the secant modulus of elasticity can be determined for any stress level. For the purposes of this investigation the secant modulus $(E)$ was determined for stress levels of 0.4 and 0.9 of the compressive strength. These values are listed in table 4.

The effect of age on the moduli of elasticity is shown in figure 3 . As with the compressive strength there is retrogression at later ages.

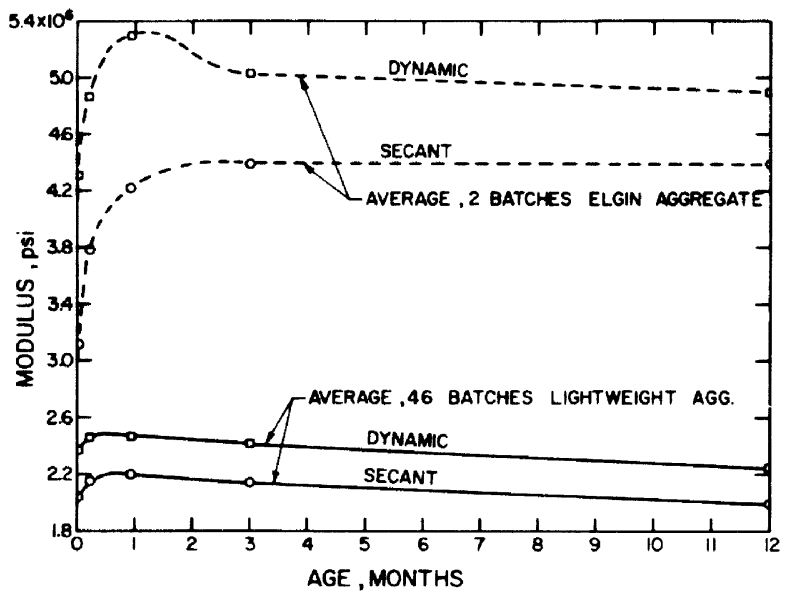

Figure 3. Average secant and dynamic modulus of elasticity for series A concretes.

The secant values are at 0.4 of the compressive strength $\left(f^{\prime} c\right)$ at the time of test.

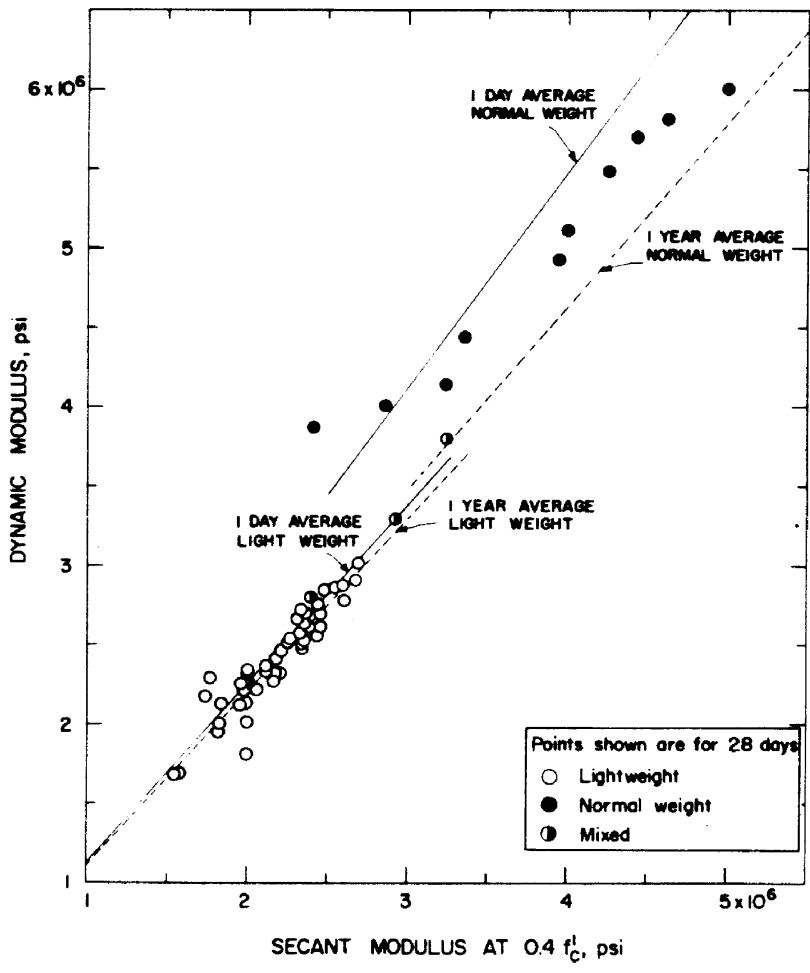

FIGURE 4. Relationship between the secant and the dynamic modulus of elasticity.

The lines shown indicate average values of the 1-day and 1-year data omitted from this graph.

TABLE 4. Moduli of elasticity of series A and series B concretes, $10^{6}$ psi

\begin{tabular}{|c|c|c|c|c|c|c|c|c|c|c|c|c|c|c|c|}
\hline \multirow{2}{*}{$\begin{array}{c}\text { Aggregate \& } \\
\text { batch }\end{array}$} & \multicolumn{5}{|c|}{$E^{2}$ at $0.4 f_{0}^{\prime 1}$} & \multicolumn{5}{|c|}{$E_{\mathrm{a}}^{2}$ at $0.9 f_{\mathrm{c}}^{\prime 1}$} & \multicolumn{5}{|c|}{$E_{\mathrm{d}}^{3}$ by Resonance method } \\
\hline & $1 \mathrm{day}$ & 7 day & 28 day & 90 day & 1 year & $1 \mathrm{day}$ & 7 day & 28 day & 90 day & 1 year & 1 day & 7 day & 28 day & 90 day & 1 year \\
\hline $\begin{array}{l}2 \mathrm{~A} \\
\mathrm{~B}_{\ldots} \ldots\end{array}$ & $\begin{array}{l}2.26 \\
2.12\end{array}$ & $\begin{array}{l}2.39 \\
2.29\end{array}$ & $\begin{array}{l}2.44 \\
2.35\end{array}$ & $\begin{array}{l}\text { 2. } 31 \\
2.23\end{array}$ & $\begin{array}{l}2.12 \\
\text { 1. } 98\end{array}$ & $\begin{array}{l}1.89 \\
1.79\end{array}$ & $\begin{array}{l}2.04 \\
2.03\end{array}$ & $\begin{array}{l}2.10 \\
2.12\end{array}$ & $\begin{array}{l}2.01 \\
2.05\end{array}$ & $\begin{array}{l}1.89 \\
1.84\end{array}$ & $\begin{array}{l}2.47 \\
2.33\end{array}$ & $\begin{array}{l}2.56 \\
2.44\end{array}$ & $\begin{array}{l}\text { 2. } 56 \\
2.53\end{array}$ & $\begin{array}{l}2.53 \\
2.41\end{array}$ & $\begin{array}{l}2.34 \\
2.17\end{array}$ \\
\hline $5 \mathrm{~A} \ldots$ & $\begin{array}{l}1.68 \\
1.71\end{array}$ & $\begin{array}{l}1.76 \\
1.78\end{array}$ & $\begin{array}{l}1.82 \\
1.83\end{array}$ & $\begin{array}{l}1.87 \\
1.70\end{array}$ & $\begin{array}{l}1.67 \\
1.73\end{array}$ & $\begin{array}{l}1.41 \\
1.41\end{array}$ & $\begin{array}{l}1.50 \\
1.52\end{array}$ & $\begin{array}{l}1.65 \\
1.62\end{array}$ & $\begin{array}{l}1.68 \\
1.59\end{array}$ & $\begin{array}{l}1.60 \\
1.67\end{array}$ & $\begin{array}{l}1.86 \\
1.93\end{array}$ & $\begin{array}{l}1.97 \\
2.00\end{array}$ & $\begin{array}{l}1.95 \\
2.00\end{array}$ & $\begin{array}{l}2.02 \\
1.95\end{array}$ & $\begin{array}{l}1.84 \\
1.86\end{array}$ \\
\hline $\begin{array}{r}6 \\
\mathbf{A} \\
\mathbf{B} \\
\mathbf{C}\end{array}$ & $\begin{array}{l}1.94 \\
2.15 \\
2.04\end{array}$ & $\begin{array}{l}1.99 \\
2.25 \\
2.00\end{array}$ & $\begin{array}{l}1.98 \\
2.34 \\
2.21\end{array}$ & $\begin{array}{l}1.85 \\
2.33 \\
2.05\end{array}$ & $\begin{array}{c}1.70 \\
1.86\end{array}$ & $\begin{array}{l}1.54 \\
1.87 \\
1.59\end{array}$ & $\begin{array}{l}1.60 \\
1.92 \\
1.69\end{array}$ & $\begin{array}{l}1.74 \\
2.10 \\
1.96\end{array}$ & $\begin{array}{l}1.67 \\
2.18 \\
2.03\end{array}$ & $\begin{array}{l}1.57 \\
1.75\end{array}$ & $\begin{array}{l}2.10 \\
2.28 \\
2.17\end{array}$ & $\begin{array}{l}2.22 \\
2.41 \\
2.28\end{array}$ & $\begin{array}{l}2.24 \\
2.47 \\
2.32\end{array}$ & $\begin{array}{l}2.12 \\
2.45 \\
2.28\end{array}$ & $\begin{array}{c}1.96 \\
2.09\end{array}$ \\
\hline 8 A ${ }_{\mathrm{B}}^{\mathrm{A}} \ldots$ & $\begin{array}{l}2.10 \\
2.29\end{array}$ & $\begin{array}{l}2.19 \\
2.38\end{array}$ & $\begin{array}{l}2.26 \\
2.42\end{array}$ & $\begin{array}{l}2.21 \\
2.53\end{array}$ & $\begin{array}{l}2.12 \\
2.32\end{array}$ & $\begin{array}{l}1.91 \\
2.00\end{array}$ & $\begin{array}{l}1.88 \\
2.00\end{array}$ & $\begin{array}{l}1.98 \\
2.16\end{array}$ & $\begin{array}{l}2.00 \\
2.27\end{array}$ & $\begin{array}{l}1.97 \\
2.18\end{array}$ & $\begin{array}{l}2.41 \\
2.57\end{array}$ & $\begin{array}{l}2.50 \\
2.69\end{array}$ & $\begin{array}{l}2.52 \\
2.76\end{array}$ & $\begin{array}{l}2.49 \\
2.77\end{array}$ & $\begin{array}{l}2.33 \\
2.53\end{array}$ \\
\hline $14 \mathrm{~A}$ & $\begin{array}{l}1.62 \\
1.78\end{array}$ & $\begin{array}{l}1.70 \\
1.76\end{array}$ & $\begin{array}{l}1.66 \\
1.76\end{array}$ & $\begin{array}{l}1.50 \\
1.77\end{array}$ & $\begin{array}{l}1.40 \\
1.64\end{array}$ & $\begin{array}{l}1.45 \\
1.60\end{array}$ & $\begin{array}{l}1.48 \\
1.58\end{array}$ & $\begin{array}{l}1.58 \\
1.70\end{array}$ & $\begin{array}{l}1.64 \\
1.83\end{array}$ & $\begin{array}{l}1.50 \\
1.70\end{array}$ & $\begin{array}{l}1.81 \\
1.95\end{array}$ & $\begin{array}{l}1.89 \\
2.01\end{array}$ & & & \\
\hline $15 \underset{\mathrm{B}_{-}}{\mathrm{A}}$ & $\begin{array}{l}1.82 \\
1.96\end{array}$ & $\begin{array}{l}1.94 \\
2.08\end{array}$ & $\begin{array}{l}1.99 \\
2.22\end{array}$ & $\begin{array}{l}1.93 \\
2.08\end{array}$ & $\begin{array}{l}1.73 \\
2.01\end{array}$ & $\begin{array}{l}1.55 \\
1.96\end{array}$ & $\begin{array}{l}1.67 \\
1.88\end{array}$ & $\begin{array}{l}1.76 \\
1.98\end{array}$ & $\begin{array}{l}1.77 \\
1.92\end{array}$ & $\begin{array}{l}1.62 \\
1.96\end{array}$ & $\begin{array}{l}2.09 \\
2.34\end{array}$ & $\begin{array}{l}2.24 \\
2.39\end{array}$ & $\begin{array}{l}2.21 \\
2.46\end{array}$ & $\begin{array}{l}2.10 \\
2.33\end{array}$ & $\begin{array}{l}1.97 \\
2.19\end{array}$ \\
\hline $16 \mathrm{~A}$ & $\begin{array}{l}2.03 \\
2.05\end{array}$ & $\begin{array}{l}2.25 \\
2.14\end{array}$ & 2.13 & $\begin{array}{l}2.08 \\
2.07\end{array}$ & $\begin{array}{l}2.03 \\
1.96\end{array}$ & $\begin{array}{l}1.80 \\
1.83\end{array}$ & $\begin{array}{l}2.04 \\
1.99\end{array}$ & 2.02 & $\begin{array}{l}2.02 \\
1.96\end{array}$ & $\begin{array}{l}\text { 1. } 97 \\
\text { 1. } 93\end{array}$ & $\begin{array}{l}2.28 \\
2.31\end{array}$ & $\begin{array}{l}2.40 \\
2.41\end{array}$ & $\begin{array}{l}2.37 \\
2.36\end{array}$ & $\begin{array}{l}2.30 \\
2.31\end{array}$ & $\begin{array}{l}2.23 \\
2.17\end{array}$ \\
\hline $17 \mathrm{~A}$ & $\begin{array}{l}2.29 \\
2.06\end{array}$ & $\begin{array}{l}2.30 \\
2.37\end{array}$ & $\begin{array}{l}2.36 \\
2.31\end{array}$ & $\begin{array}{l}2.42 \\
2.31\end{array}$ & $\begin{array}{l}2.14 \\
2.28\end{array}$ & $\begin{array}{l}1.94 \\
2.06\end{array}$ & $\begin{array}{l}1.97 \\
2.08\end{array}$ & $\begin{array}{l}2.08 \\
2.06\end{array}$ & $\begin{array}{l}2.27 \\
2.13\end{array}$ & $\begin{array}{l}2.05 \\
2.21\end{array}$ & $\begin{array}{l}2.56 \\
2.58\end{array}$ & $\begin{array}{l}2.66 \\
2.66\end{array}$ & $\begin{array}{l}2.64 \\
2.66\end{array}$ & $\begin{array}{l}2.57 \\
2.62\end{array}$ & $\begin{array}{l}2.39 \\
2.50\end{array}$ \\
\hline $18 \mathrm{~A}$ & $\begin{array}{l}2.04 \\
2.06\end{array}$ & $\begin{array}{l}2.13 \\
1.98\end{array}$ & $\begin{array}{l}2.16 \\
2.17\end{array}$ & $\begin{array}{l}2.15 \\
2.00\end{array}$ & $\begin{array}{l}1.90 \\
1.90\end{array}$ & $\begin{array}{l}1.81 \\
1.91\end{array}$ & $\begin{array}{l}1.98 \\
1.89\end{array}$ & $\begin{array}{l}2.00 \\
2.02\end{array}$ & $\begin{array}{l}2.06 \\
1.94\end{array}$ & $\begin{array}{l}1.87 \\
1.88\end{array}$ & $\begin{array}{l}2.19 \\
2.18\end{array}$ & $\begin{array}{l}2.30 \\
2.25\end{array}$ & $\begin{array}{l}2.27 \\
2.31\end{array}$ & $\begin{array}{l}2.20 \\
2.22\end{array}$ & $\begin{array}{l}2.10 \\
2.14\end{array}$ \\
\hline
\end{tabular}


TABLE 4. Moduli of elasticity of series A and series B concretes, $10^{6}$ psi-Continued

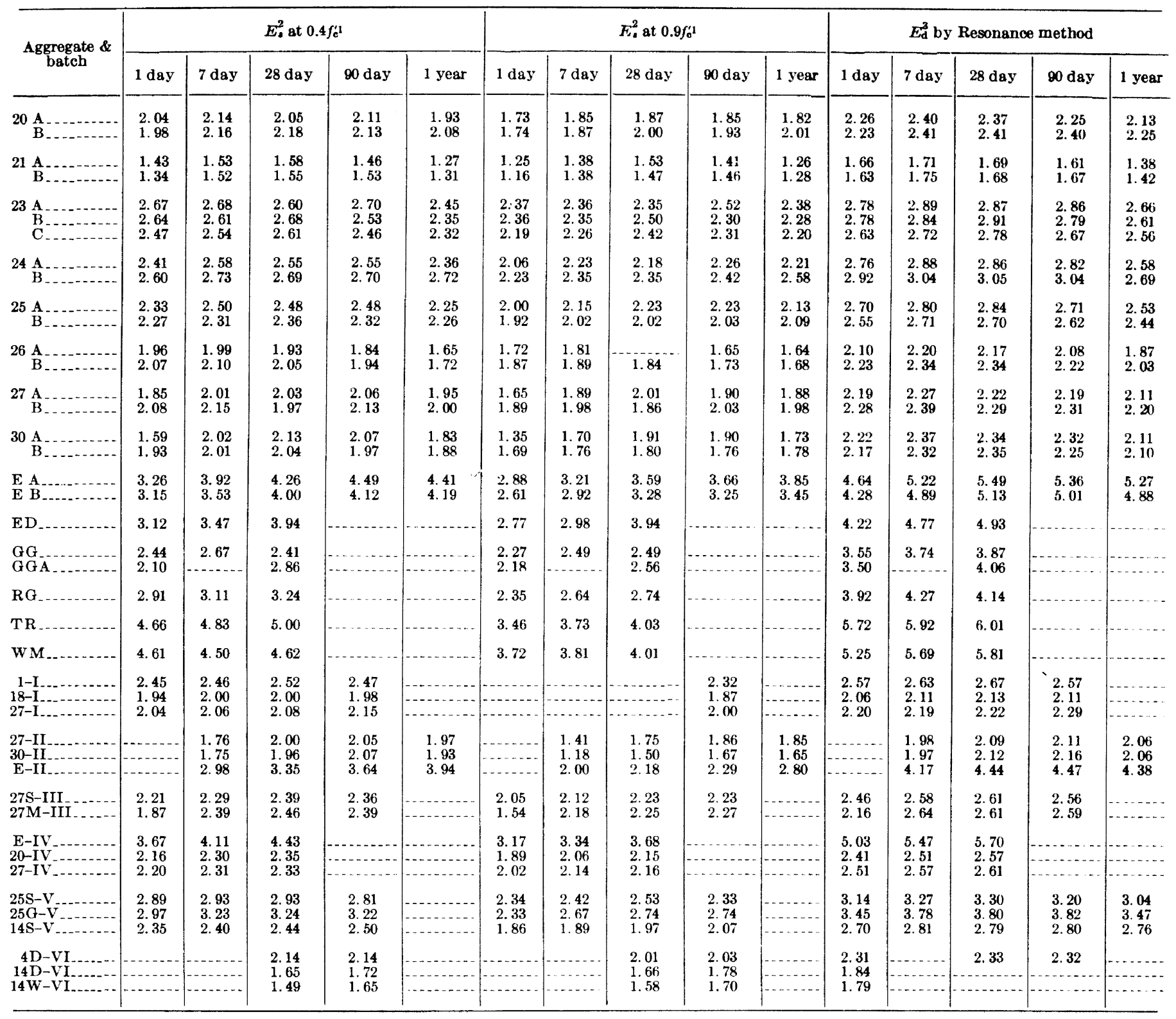

$1 f_{c}^{\prime}=$ Compressive strength at age tested.

${ }_{3} E_{s}=$ Secant modulus of elasticity.

$3 E_{d}=$ Dynamic modulus from the longitudinal resonaner frequency

The similarity between the dynamic and secant modulus curves is rather close for both types of concrete.

A direct comparison of the dynamic with the secant values of modulus, shown in figure 4 , indicates that the ratio of the moduli is higher for the normal weight concretes than for the lightweight. The average ratio of the dynamic to the secant modulus at the age of 28 days was 1.11 for the lightweight and 1.32 for the normal-weight concretes. This ratio decreased with age for both types of concrete. For the one-day test these ratios were 1.13 and 1.37 for the lightweight and normal weight respectively. For the 1-year test the ratios were 1.10 and 1.16 .

Poisson's ratio, which was determined only by the resonance method, varied little with age, strength or aggregate except for a slight drop in value after 90 days. For the lightweight concretes the individual values of the ratio varied from 0.16 to 0.25 with the average being 0.21 . The average Poisson's ratio for the normal-weight concretes was 0.20 with individual values ranging from 0.16 to 0.23 .

\subsubsection{Results of Flexural Strength Tests}

The average flexural-strength age data, which are shown graphically in figure 1, illustrate one difference between the lightweight and normalweight concretes. Examination of figure 1 and table 5 will show that air-dried specimens of lightweight concrete have significantly lower flexural strengths than moist specimens, but for the normal-weight concrete the opposite is true. 
TABLE 5. ReBults of flexural strength test

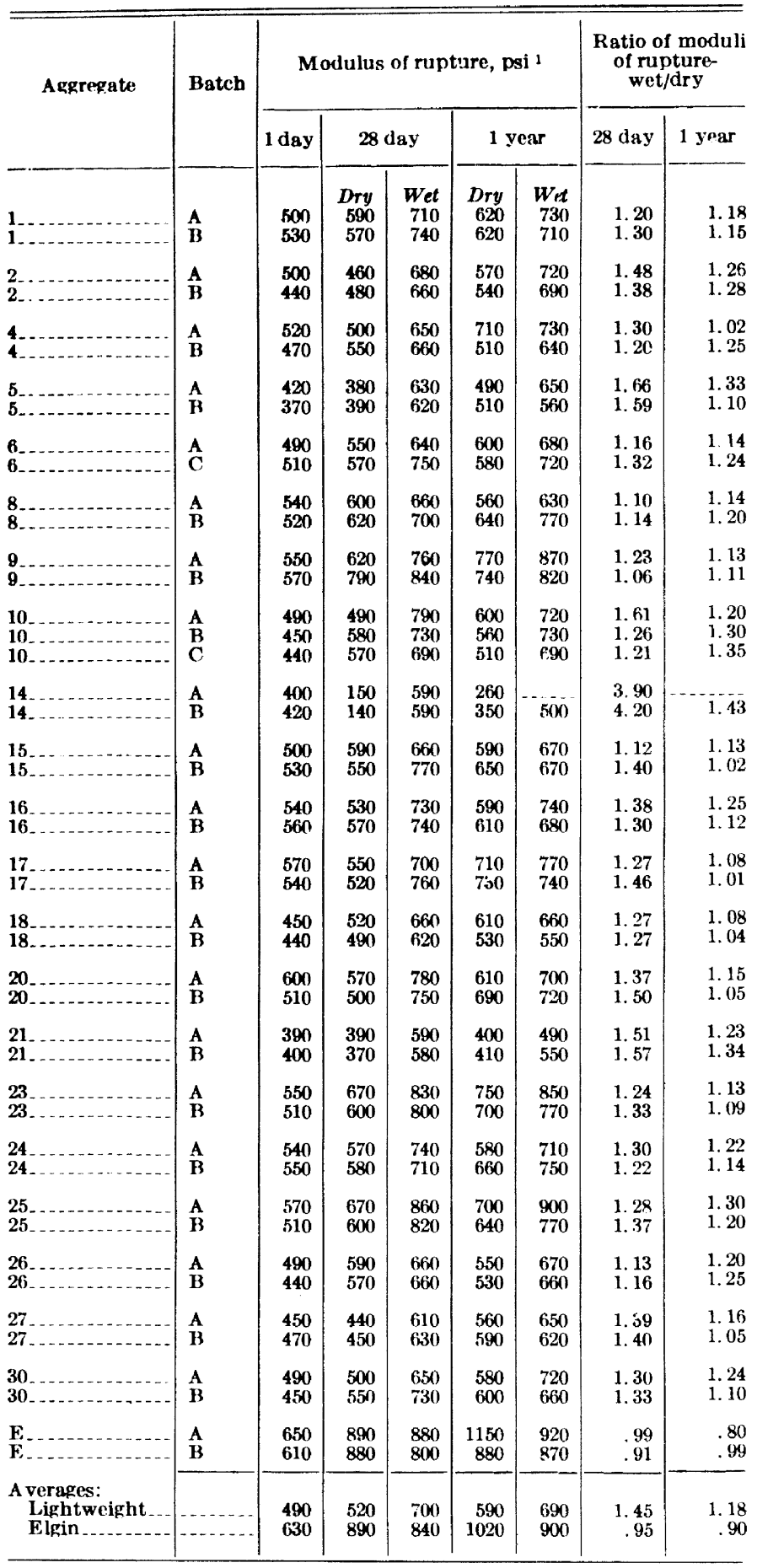

${ }^{1}$ Each value is average of 4 breaks on 2 specimens.

This observation is similar to the results reported by Hanson [5] for the indirect tension test on concretes made from aggregate $E$. Other investigators $[6,7]$, on the contrary, have reported results which indicated lower flexural strengths for dry than for continuously moist normal-weight concretes. This apparent conflict among the findings of different investigators may be a reflection of the intrinsic differences in the aggregates used.

Differences between concretes made from different lightweight aggregates can be illustrated by comparing the flexural-strength data for aggregate 14 with those for the other lightweight aggregates in table 5. The drying of aggregate 14 specimens reduced the flexural strength to a much greater extent than for the other lightweights, especially for the 28-day test. Shrinkage cracks became visible on all air-dried test specimens including the loaded creep specimens of concretes made from this aggregate ${ }^{4}$ when they were about 3 weeks old, but none were visible on specimens of the other concretes. These shrinkage cracks were no longer visible on the loaded creep specimens after 1-year drying, but were still visible on the unloaded specimens.

The average flexural strength of lightweight concrete kept damp until tested at 28 days was 45 percent greater than that of the air-dried concrete. This advantage decreased to 18 percent at 1 year due to the fact that there was no increase in the flexural strength of the moist-cured specimens while the dry flexural strength increased.

There does not appear to be any direct correlation between the flexural and the compressive strengths of the lightweight concretes for the range in values found in this investigation.

\subsubsection{Creep and Shrinkage}

The creep and shrinkage characteristics of a concrete are very important in the design of any concrete structural member which will be under a sustained load. These characteristics are important since the deformation of the member with time can be three or more times as great as the elastic deformation occurring upon application of the load.

For design purposes the creep of a concrete is usually considered separately from the drying shrinkage, since the amount of the creep is dependent on the magnitude of the load. The term "creep plus shrinkage," as used in this report, is the deformation, exclusive of the elastic deformation, of the test specimen under the sustained compressive load. Although it is thought [8] that the drying shrinkage of the concrete specimen is affected by the load, the term "shrinkage" is taken here as the shortening of the companion, unloaded specimen, while "creep" is taken as the difference between the deformations of the loaded and unloaded specimens.

The shrinkage values reported here are assumed to be a combination of drying and some carbonation shrinkage. It has been shown by Verbeck [9] and others that carbonation effects are at a maximum when the relative humidity is about 50 percent - the storage conditions for the shrinkage and creep specimens in this investigation. The effect of carbonation on the creep of concrete is not known.

Creep and shrinkage data for the series A concretes are given in table 6 and figure 5 . The "adjusted creep" values given in this table were

4 The fines of this aggregate, which were used in the series $A$ tests, are seldom used. A discussion of the results obtained from concretes made from this aggregate, which is considered to be quite different than the other lightweight aggregates, is given in the appendix. 

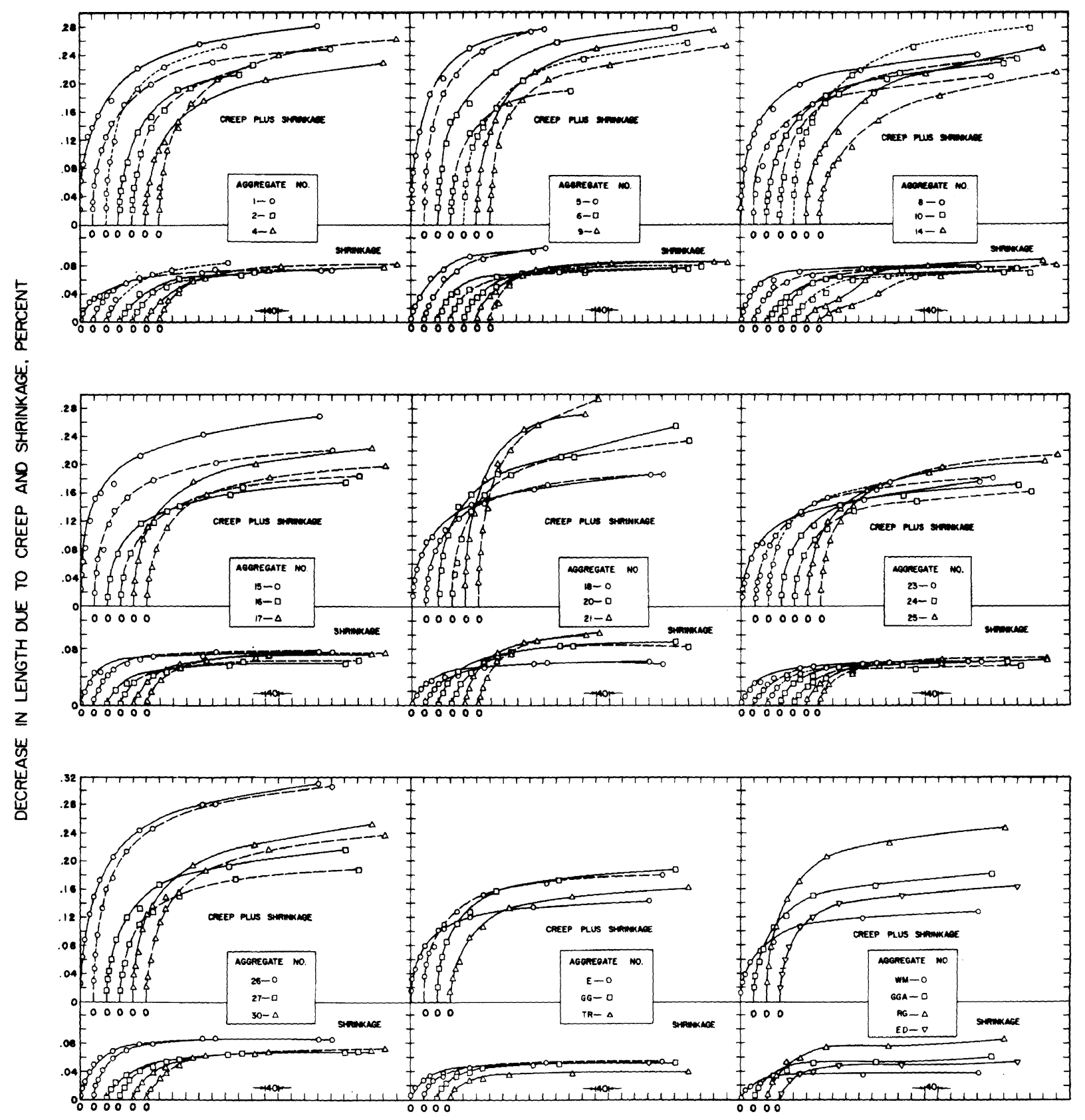

TME, DAYS

FiguRe 5. Creep and shrinkage data for series A concretes.

Different types of lines indicate different batches. The plotted values are the average of 2 specimens.

adjusted to a stress-strength ratio of 0.5 by assuming that the creep was proportional to the ratio of the sustained stress to the compressive strength of the concrete at the time of loading. This adjustment of the measured creep values was made necessary because, although the creep stress on all the series A concretes was 2000 psi, the compressive strengths of the concretes varied above and below the nominal $4000 \mathrm{psi}$. The adjusted values are thought to be better than the measured values as a basis for comparison of the various concretes. Neville [10] has presented data which justified this use of the stress-strength ratio.
The creep-rate factor, $F(k)$, given in table 6 and used in estimating the 20-year creep values for these concretes is the factor in the empirical formula $\epsilon=F(k) \log _{e}(t+1)$ where $\epsilon$ is the creep at time, $t$, in days. This formula is specified in the proposed ASTM creep test method and is used by the Bureau of Reclamation [11] and others. Although this formula does not accurately describe the data at early ages (less than 28 days), it was used because the fit was satisfactory at later ages. Although this formula has been shown to be fairly reliable under certain circumstances, the reliability of the 20-year creep values estimated from this 
TABLE 6. Creep and shrinkage data for series A concretes

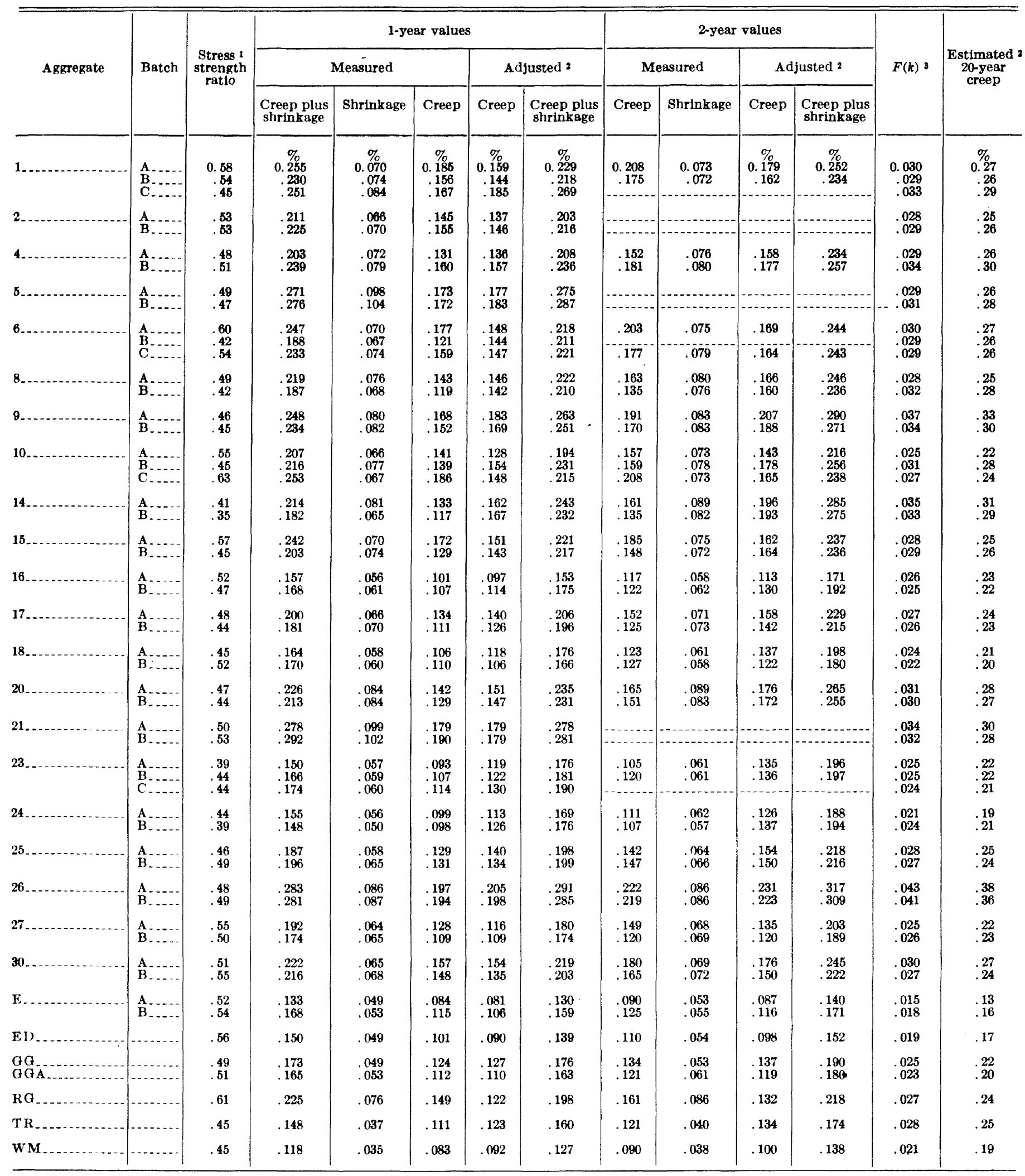

1 Stress $=2000 \mathrm{psi}$ creep load; strength $=$ compressive strength, psi, at the time of creep loading.

${ }_{3}^{2}$ Creep adjusted to the stress-strength ratio of 0.50 . Where $\epsilon=$ creep in percent for stress-strength ratio of 0.50 at time $(t)$ in days since loading. $F(k)$ is the slope of the line drawn through a semilog plot of the creep data.
s. 


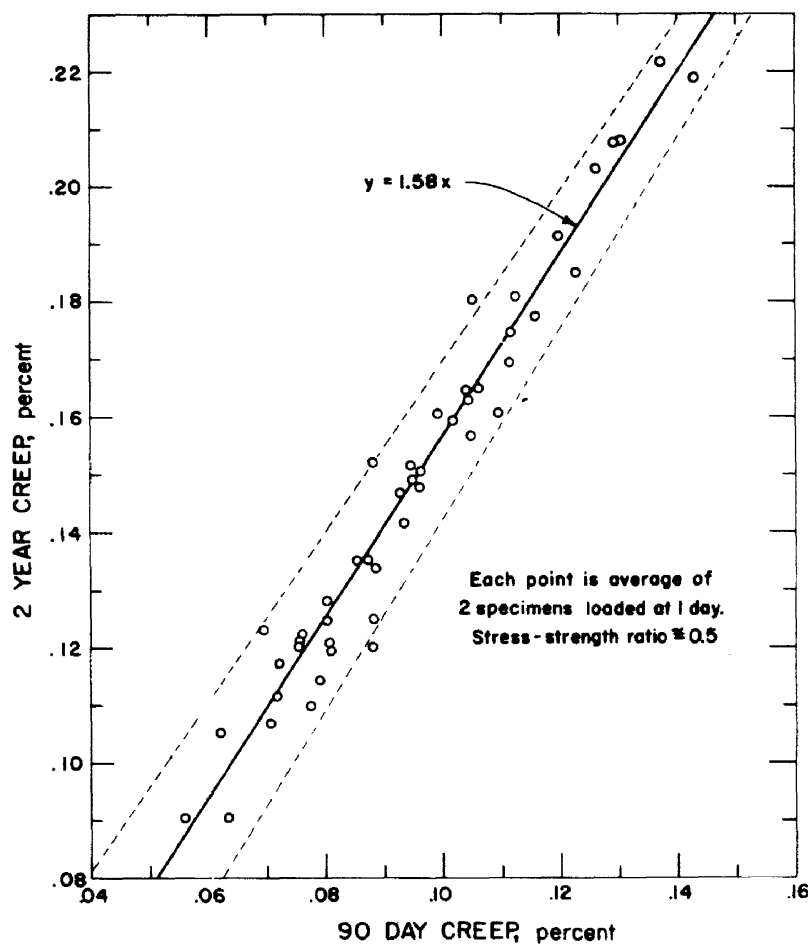

FiguRe 6. Relationship between creep at 90 days and at two years for series A concretes.

Stress-strength ratio is the ratio of the sustained creep stress (2000 psi) to the strength of the concrete at the time of loading (approximately $4000 \mathrm{psi}$ ).

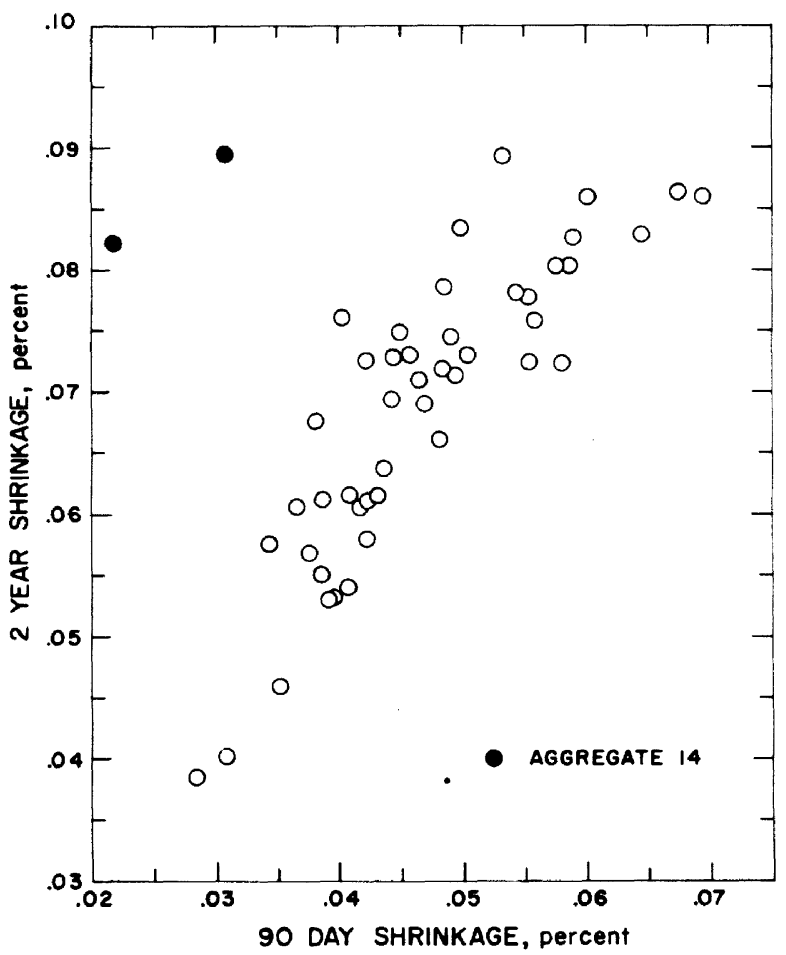

Figure 7. Relationship between shrinkage at 90 days and at two years for series A concretes. formula should be considered questionable at this point.

Because of the nature of the phenomena, investigations of creep and shrinkage constitute long-term studies, and several relationships were investigated with the view of finding one which might be useful for predicting creep. Figures 6 and 7 were plotted with the thought that there might be a fairly reliable relationship between early creep and shrinkage data and results at later ages. The average line through the data in figure 6 indicates that the average value of creep for concretes at 2 years is about 1.6 times the creep at 90 days. For comparison, the empirical formula given above estimates that the creep at 2 years would be about 1.5 times the creep at 90 days.

Extrapolating beyond the data shown as figure 6 by assuming the creep-time curve is logarithmic, it is estimated that the creep at 20 years will be about 1.4 times the 2 -year value or 2.2 times the 90-day value.

The correlation between the 90 day and 2 year shrinkage was not as well defined as that for the creep data. The shrinkage data, plotted as figure 7 , shows a considerable scatter, with the data for the concretes made from aggregate $14^{5}$ showing a particularly large divergence from the other concretes.

The creep and shrinkage data for the series A concretes are plotted as histograms in figure 8 . These histograms provide a convenient means of comparing the creep and shrinkage values of light-
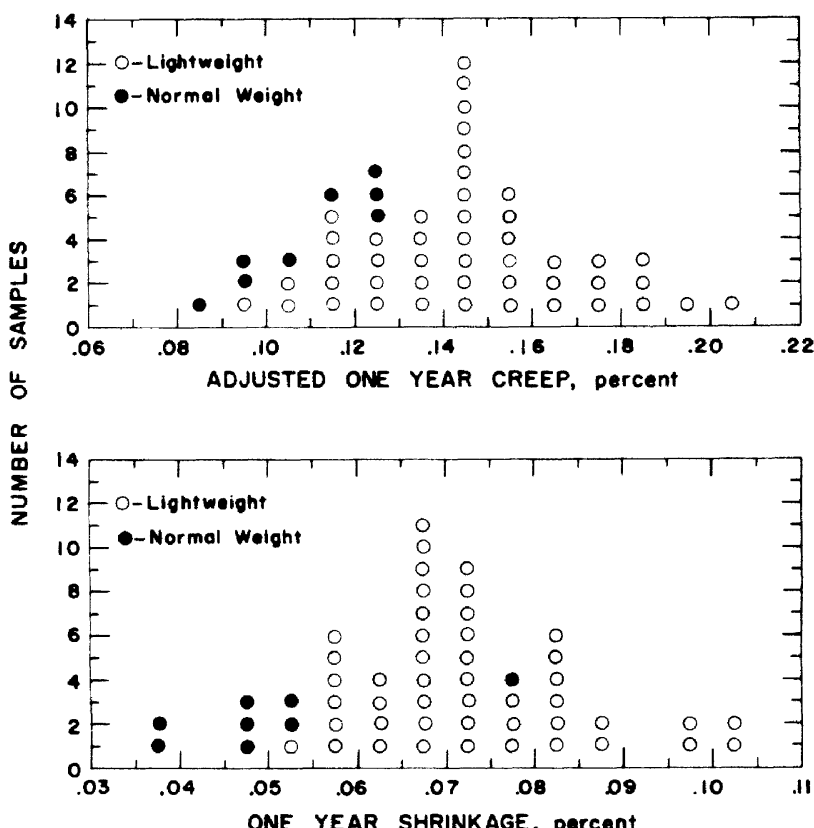

Figure 8. Frequency histograms of creep and shrinkage data for series A concretes.

The creeps were adjusted to a stress-strength ratio of 0.5 , by assuming that the creep was proportional to the ratio of the sustained stress to the compressive strength of the concrete at the time of loading.

${ }^{6}$ It will be recalled that concrete with aggregate 14 developed visible shrinkage cracks in air-dried specimens at an age of 3 weeks. 


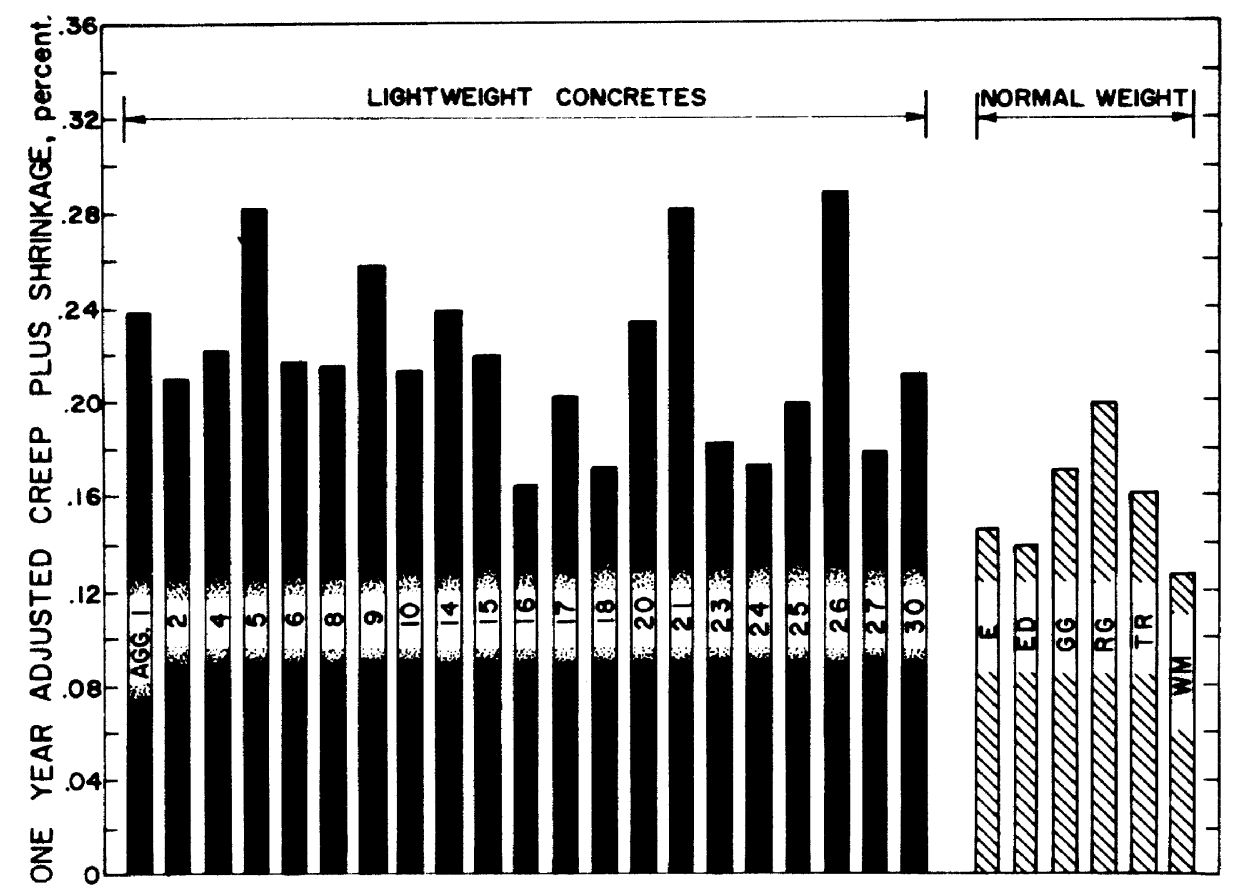

FIGURE 9. Bar chart showing the adjusted creep plus shrinkage for each aggregate at one year.

The creeps were adjusted to a stress-strength ratio of 0.5 . Values shown are an average of 2 specimens from each of at least 2 batches.

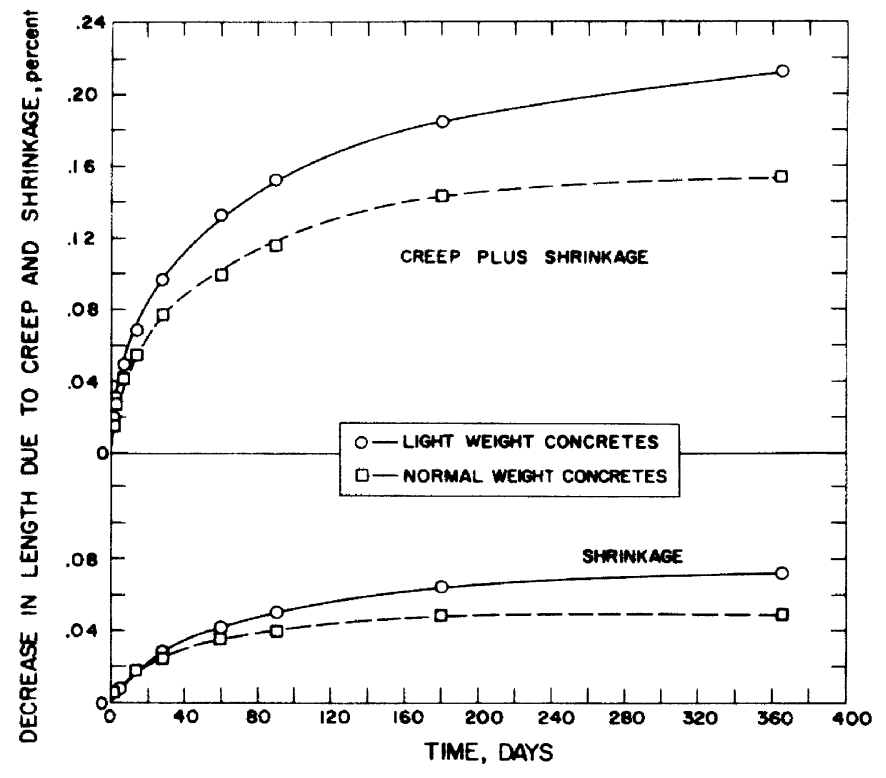

FiguRE 10. Creep-and shrinkage-time data for the average series A lightweight and normal-weight concretes.

The values shown are averages for 4 i hatches of lightweight and for 8 batches of normal-weight concretes.

weight and normal-weight concretes. As can be seen, there is considerable overlapping of the data, but in general the normal-weight concretes exhibited less creep and shrinkage than the lightweight concretes. However, some lightweights exhibited less creep and shrinkage than some normal-weight concretes. A bar chart comparison of the creep plus shrinkage of the concretes made from each aggregate is given as figure 9 .

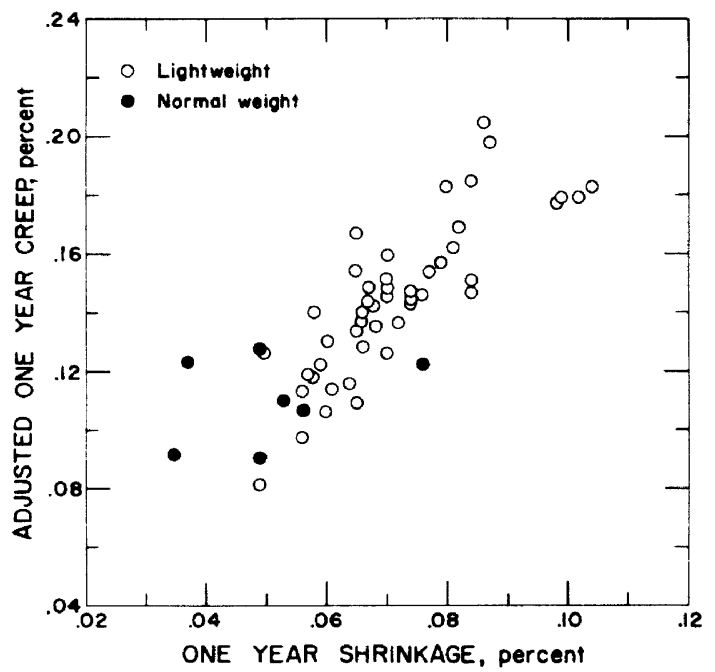

FIGURE 11. Relationship between the creep and the shrinkage values at one year.

The creep values were adjusted to a stress-strength ratio of 0.5

The average adjusted creep of the lightweight concretes was 0.145 percent at 1 year, while the average for the normal-weight concretes was 0.106 percent. The average 1-year shrinkages of the lightweight and normal-weight concretes were 0.072 percent and 0.050 percent, respectively. The grand average creep and shrinkage curves for the lightweight and normal-weight concretes are shown in figure 10.

Figure 11 indicates a rough correlation between the adjusted creep and the shrinkage of a concrete observed in specimens 1 year old.

Figure 12 shows the relationship between the 
TABLE 7. Test data for additional series A concretes under test for less than 1 year

TABLE 7A. Mix proportions, unit weight, and strength ${ }^{1}$

\begin{tabular}{|c|c|c|c|c|c|c|c|c|c|c|c|c|c|c|c|c|c|}
\hline \multirow{3}{*}{ Aggregate } & \multirow{3}{*}{ Batch } & \multicolumn{4}{|c|}{ Quantities per cu yd } & \multirow{3}{*}{$\begin{array}{c}\text { Air } \\
\text { content }\end{array}$} & \multirow{3}{*}{ Slump } & \multicolumn{4}{|c|}{ Unit weight } & \multicolumn{2}{|c|}{$\begin{array}{c}\text { Aggregate } \\
\text { moisture when } \\
\text { batched }\end{array}$} & \multicolumn{4}{|c|}{ Compressive strength } \\
\hline & & \multirow{2}{*}{ Water } & \multirow{2}{*}{ Cement } & \multicolumn{2}{|c|}{ Aggregate } & & & \multirow{2}{*}{ Plastic } & \multirow{2}{*}{1 day } & \multirow{2}{*}{28 day } & \multirow{2}{*}{$\begin{array}{l}\text { Oven } \\
\text { dry }\end{array}$} & \multirow{2}{*}{ Fine } & \multirow{2}{*}{ Coarse } & \multirow{2}{*}{1 day } & \multirow{2}{*}{7 day } & \multirow{2}{*}{28 day } & \multirow{2}{*}{90 day } \\
\hline & & & & Fine & Coarse & & & & & & & & & & & & \\
\hline $\begin{array}{l}11 \ldots \\
22 \ldots \\
28 . . .\end{array}$ & $\begin{array}{l}\text { A } \\
\text { B } \\
\text { A } \\
\text { B } \\
\text { A } \\
\text { B }\end{array}$ & $\begin{array}{r}l b \\
452 \\
484 \\
508 \\
495 \\
\mathbf{4 6 2} \\
\mathbf{4 3 1}\end{array}$ & $\begin{array}{r}l b \\
660 \\
623 \\
651 \\
668 \\
600 \\
588\end{array}$ & $\begin{array}{c}l b \\
1030 \\
1030 \\
907 \\
900 \\
801 \\
782\end{array}$ & $\begin{array}{r}l b \\
836 \\
836 \\
620 \\
615 \\
884 \\
867\end{array}$ & $\begin{array}{l}\% \\
3.5 \\
3.5 \\
3.5 \\
3\end{array}$ & $\begin{array}{l}\text { in. } \\
\text { 3. } \\
3.0 \\
2.0 \\
2.5 \\
4.0 \\
\text { 3. } 0\end{array}$ & \begin{tabular}{r}
\multicolumn{1}{c}{$p c f$} \\
108.4 \\
108.0 \\
98.2 \\
99.0 \\
99.4 \\
96.0
\end{tabular} & \begin{tabular}{r}
\multicolumn{1}{c}{$p c f$} \\
110.3 \\
110.0 \\
98.1 \\
99.1 \\
101.6 \\
98.5
\end{tabular} & $\begin{array}{r}p c f \\
105.6 \\
105.6 \\
91.5 \\
93.0 \\
97.0 \\
94.0\end{array}$ & $\begin{array}{r}p c f \\
99.9 \\
100.2 \\
85.6 \\
87.3 \\
90.3 \\
87.8\end{array}$ & $\begin{array}{r}\% \\
3.2 \\
3.2 \\
.4 \\
.4 \\
.2 \\
.2\end{array}$ & $\begin{array}{r}\% \\
1.6 \\
1.6 \\
.1 \\
.1 \\
.4 \\
.4\end{array}$ & $\begin{array}{c}p s i \\
4700 \\
4690 \\
3880 \\
4530 \\
4490 \\
3870\end{array}$ & $\begin{array}{c}p s i \\
5830 \\
5810 \\
4590 \\
4840 \\
5710 \\
5140\end{array}$ & $\begin{array}{c}\text { psi } \\
6800 \\
6750 \\
5300 \\
5300 \\
7250 \\
6000\end{array}$ & $\begin{array}{c}p 8 i \\
7040 \\
6730 \\
5330 \\
5190 \\
7040 \\
6630\end{array}$ \\
\hline
\end{tabular}

TABLE 7B. Moduli of elasticity, ${ }^{2} 10^{6}$ psi

\begin{tabular}{|c|c|c|c|c|c|c|c|c|c|c|c|c|c|}
\hline \multirow{2}{*}{ Aggregate } & \multirow{2}{*}{ Batch } & \multicolumn{4}{|c|}{$\mathrm{E}_{\mathrm{B}}$ at $0.4 \mathrm{f}^{\prime} \mathrm{c}$} & \multicolumn{4}{|c|}{$\mathrm{E}_{\mathrm{a}}$ at $0.9 \mathrm{f}^{\prime} \mathrm{c}$} & \multicolumn{4}{|c|}{$\mathbf{E}_{d}$ by resonance method } \\
\hline & & 1 day & 7 day & 28 day & 90 day & 1 day & 7 day & 28 day & 90 day & 1 day & 7 day & 28 day & 90 day \\
\hline $\begin{array}{l}11 . \\
22 \\
28\end{array}$ & $\begin{array}{l}\text { A } \\
\text { B } \\
\text { A } \\
\text { B } \\
\text { A } \\
\text { B }\end{array}$ & $\begin{array}{l}2.04 \\
2.06 \\
1.43 \\
1.63 \\
2.16 \\
1.93\end{array}$ & $\begin{array}{l}2.16 \\
2.23 \\
1.67 \\
1.77 \\
2.18 \\
2.06\end{array}$ & $\begin{array}{l}2.31 \\
2.09 \\
1.85 \\
1.80 \\
2.25 \\
2.11\end{array}$ & $\begin{array}{l}2.24 \\
2.19 \\
1.69 \\
1.77 \\
2.20 \\
2.07\end{array}$ & $\begin{array}{l}1.61 \\
1.65 \\
1.30 \\
1.46 \\
1.86 \\
1.71\end{array}$ & $\begin{array}{l}1.85 \\
1.83 \\
1.61 \\
1.61 \\
1.92 \\
1.77\end{array}$ & $\begin{array}{l}2.10 \\
1.88 \\
1.86 \\
1.68 \\
2.00 \\
1.86\end{array}$ & $\begin{array}{l}2.03 \\
1.98 \\
1.63 \\
1.64 \\
2.02 \\
1.86\end{array}$ & $\begin{array}{l}2.60 \\
2.60 \\
1.85 \\
2.01 \\
2.36 \\
2.20\end{array}$ & $\begin{array}{l}2.70 \\
2.72 \\
1.99 \\
2.04 \\
2.43 \\
2.33\end{array}$ & $\begin{array}{l}2.73 \\
2.75 \\
2.00 \\
2.06 \\
2.51 \\
2.33\end{array}$ & $\begin{array}{l}2.67 \\
2.69 \\
1.97 \\
2.06 \\
2.43 \\
2.30\end{array}$ \\
\hline
\end{tabular}

TABLE 7C. Flexural strength, creep, and shrinkage ${ }^{3}$

\begin{tabular}{|c|c|c|c|c|c|c|c|c|c|c|c|c|}
\hline \multirow{4}{*}{ Aggregate } & \multirow{4}{*}{ Batch } & \multicolumn{4}{|c|}{ Flexural strength test } & \multicolumn{3}{|c|}{ 90-day measured values } & \multirow{4}{*}{$\begin{array}{l}\text { Stress- } \\
\text { strength } \\
\text { ratio }\end{array}$} & \multirow{4}{*}{$\begin{array}{l}\text { Adjusted } \\
90 \text {-day } \\
\text { creep }\end{array}$} & \multicolumn{2}{|c|}{ Estimated creep } \\
\hline & & \multicolumn{3}{|c|}{ Modulus of rupture, psi } & \multirow{3}{*}{$\frac{\begin{array}{c}\text { Ratio, wet- } \\
\text { dry }\end{array}}{28 \text { day }}$} & \multirow{3}{*}{$\begin{array}{c}\text { Creep } \\
\text { plus } \\
\text { shrinkage }\end{array}$} & \multirow{3}{*}{ Shrinkage } & \multirow{3}{*}{ Creep } & & & & \\
\hline & & \multirow{2}{*}{1 day } & \multicolumn{2}{|c|}{28 day } & & & & & & & 2 уеал & year \\
\hline & & & Dry & Wet & & & & & & & & \\
\hline $\begin{array}{l}11 \ldots \\
22 \ldots \\
28\end{array}$ & $\begin{array}{l}\mathbf{A} \\
\mathbf{B} \\
\mathbf{A} \\
\mathbf{B} \\
\mathbf{A} \\
\mathbf{A} \\
\mathbf{B}\end{array}$ & $\begin{array}{l}p s i \\
560 \\
510 \\
480 \\
440 \\
530 \\
490\end{array}$ & $\begin{array}{l}p s i \\
630 \\
580 \\
480 \\
410 \\
600 \\
570\end{array}$ & $\begin{array}{l}p s i \\
820 \\
760 \\
660 \\
650 \\
690 \\
710\end{array}$ & $\begin{array}{l}1.30 \\
1.31 \\
1.38 \\
1.59 \\
1.15 \\
1.25\end{array}$ & $\begin{array}{r}\% \\
0.175 \\
.169 \\
.182 \\
.162 \\
.161 \\
.180\end{array}$ & $\begin{array}{r}\% \\
0.055 \\
.054 \\
.061 \\
.063 \\
.052 \\
.054\end{array}$ & $\begin{array}{l}\% \\
0.120 \\
.115 \\
.121 \\
.099 \\
.109 \\
.126\end{array}$ & $\begin{array}{l}\% \\
43 \\
43 \\
52 \\
44 \\
45 \\
52\end{array}$ & $\begin{array}{l}\% \\
0.140 \\
.134 \\
.116 \\
.113 \\
.121 \\
.121\end{array}$ & $\begin{array}{l}\% \\
0.22 \\
.21 \\
.18 \\
.18 \\
.19 \\
.19\end{array}$ & $\begin{array}{r}\% \\
0.31 \\
.29 \\
.26 \\
.25 \\
.27 \\
.27\end{array}$ \\
\hline
\end{tabular}

1 See footnotes for table 3 .

2 See footnotes for table 4 .

${ }^{3}$ See footnotes for tables 5 and 6 .

4 -year creep $=1.6$ times 90 -day adjusted creep. 20 -year creep $=2.2$ times 90 -day adjusted creep.

cement contents of some series A concretes and their "creep plus shrinkage "values. Data for all series A concretes which had 1-day strengths between 3,800 and 4,000 psi were used in this plot so that no adjustment of creep for stress-strength ratio was needed. Although the data show considerable scatter, this graph indicates that in general the greater the amount of cement needed to develop a given strength, the greater is the "creep plus shrinkage". Lyse presented some date [12] which also indicates that creep and shrinkage may be proportional to the percentage of cement paste in a concrete.

\subsubsection{Moisture Expansion}

The possibility of an expansive reaction between an aggregate and the cement or even long-term moisture expansion of the aggregate itself was investigated by determining the length change with time of a specimen of each concrete stored in the fog room.

After about 1 year of storage in the fog room, the maximum expansion recorded was 0.014 percent. The average expansion of all the concretes was 0.007 percent. An average increase in weight of 1.7 percent was recorded. The maximum increase in weight was 2.3 percent. There was a very slight increase in expansion, and weight for a $2 \mathrm{~d}$ year of exposure.

\subsection{Results-Series B Tests}

A series of supplemental tests was designed to investigate certain variables within the series A tests described in the preceding section. These supplemental tests, six of which are described in 


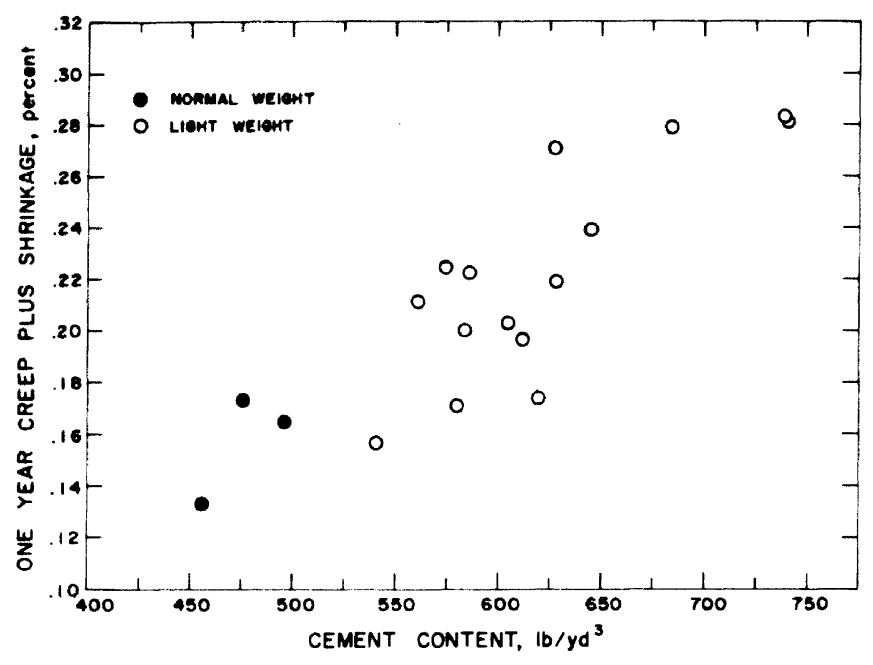

FIGURE 12. Relationship between creep plus shrinkage and the cement content.

The values shown are for series A concretes with a $24 \mathrm{hr}$ strength between 3800 and 4200 psi.

this report, were called the series $B$ tests. The data from these six tests are given in tables 8 through 14 with the test number being designated by Roman numerals. Because a correlation with series A data was necessary, the mix design, test procedure, etc., of series $A$ were followed in the series B tests where applicable. Significant mix data for the series B concretes are given in table 8 .

\subsubsection{Supplemental Test I}

Test I was designed to develop data on the effect of the type of cement on the length change characteristics of steam-cured concretes. The concretes for this test were designed to have the same cement content and strength at the time of loading as those of series A, except that they were to be made with a type I cement, steam-cured 36 $\mathrm{hr}$, and placed under load when 2 days old. The concretes were loaded in creep frames at three stress levels, $1,000,2,000$, and 2,500 psi.
Table 9 gives 2,000 psi creep load data obtained for test I and series A concretes made with three aggregates. These data indicate that the concretes made with the type I cement exhibit less creep and drying shrinkage then the concretes made with the type III cement. The reductions in adjusted creep for aggregates 18 and 27 due to the use of type I cement were 17 and 10 percent, respectively. The reduction of 36 percent observed for aggregate 1 was probably due to some extent to the large difference in the slumps of the concretes.

\subsubsection{Supplemental Test II}

The concretes for test II were designed to be representative of mixes used in ordinary in situ concrete work with a 28-day compressive strength of 3,000 psi. These concretes were made with the type I cement, damp cured for 6 days, and placed under creep test at loads of 600 and 1,200 psi when 7 days old. Creep test data for these concretes stressed to $1,200 \mathrm{psi}$ and concretes of series $A$ and test I made from the same aggregates are given in table 10 .

Although the cement contents of the test II concretes were less, the 1 year creep data for comparable concretes of series $\mathrm{A}$ are similar when compared on the basis of equal stress-strength ratio of 0.5 , except for aggregate 30 concretes. The concretes of aggrega te 30 differed considerably in the slump, stress-strength ratio, as well as the cement content.

A comparison of the data for the aggregate 27 concretes of test II and test I indicates that the amount of creep for the moist-cured and steamcured concretes are about the same. The data for the aggregate 30 concretes appear to indicate a considerable effect of slump on creep and shrinkage as mentioned in the previous paragraph. This effect is similar to that noted in test I for aggregate 1 concretes. However, the data for the concretes

TABLE 8. Data for series B concretes

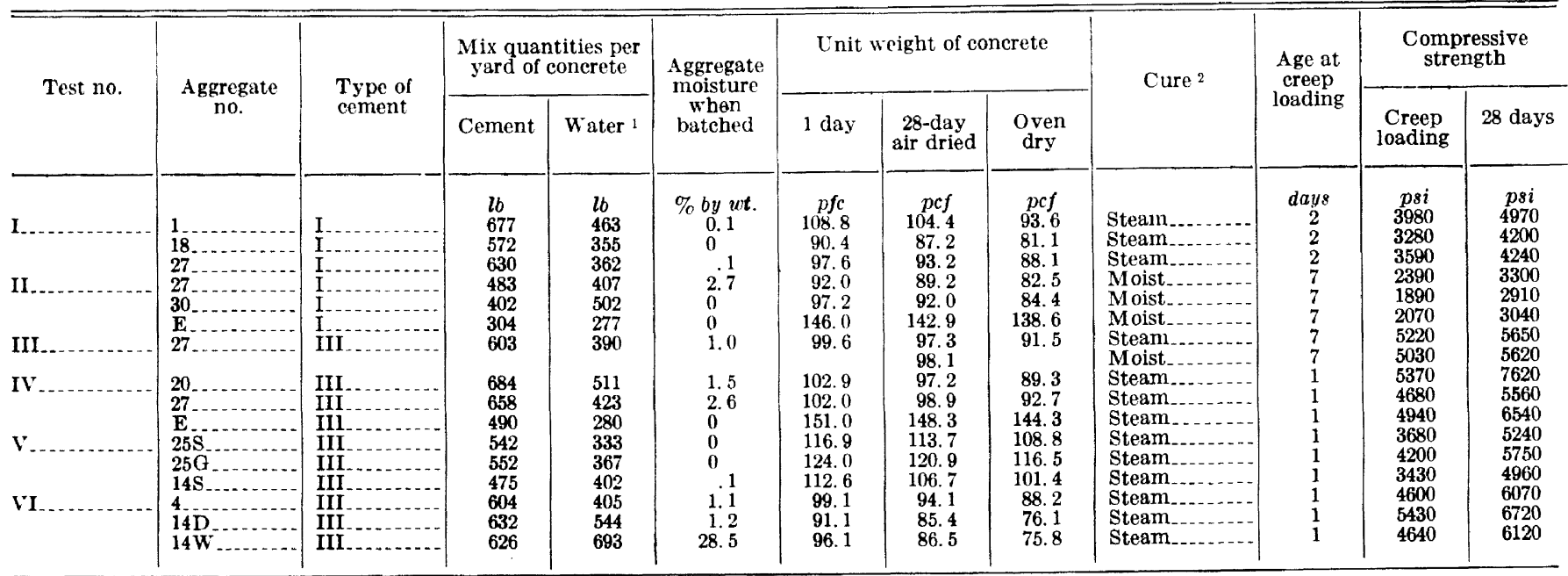

1 Includes moisture in aggregate when batched.

2 Steam-cured concretes made from type I cement were steam-cured 36 hours, those made from type III were steam-cured 12 hours. 
TABLE 9. Effect of type of cement on creep and shrinkage of steam-cured concretes

\begin{tabular}{|c|c|c|c|c|c|c|c|c|c|c|}
\hline \multirow[b]{2}{*}{ Test } & \multirow[b]{2}{*}{ Aggregate } & \multirow{2}{*}{$\begin{array}{l}\text { Type of } \\
\text { cement }\end{array}$} & \multirow[b]{2}{*}{ Slump } & \multirow{2}{*}{$\begin{array}{l}\text { Cement } \\
\text { content }\end{array}$} & \multirow{2}{*}{$\begin{array}{l}\text { Stress- } \\
\text { strength } \\
\text { ratio }\end{array}$} & \multirow{2}{*}{$\begin{array}{l}\text { 28-day } \\
\text { strength }\end{array}$} & \multicolumn{3}{|c|}{ Measured values at 1 year } & \multirow{2}{*}{$\begin{array}{l}\text { Adjuste } \\
\text { creep } 1 \text { at } \\
1 \text { year }\end{array}$} \\
\hline & & & & & & & $\begin{array}{c}\text { Shrink- } \\
\text { age }\end{array}$ & $\begin{array}{l}\text { Creep } \\
\text { plus } \\
\text { shrink- } \\
\text { age }\end{array}$ & Creep & \\
\hline $\begin{array}{l}\text { I } \\
\text { Series A } \\
\text { I. } \\
\text { I } \\
\text { Series A } \\
\end{array}$ & $\begin{array}{c}1 \ldots \\
18 . \\
18 . \\
18 \ldots \\
27 . \ldots \\
27 .\end{array}$ & 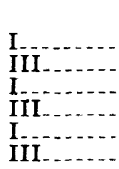 & $\begin{array}{l}\text { in. } \\
1.5 \\
3.0 \\
2.0 \\
2.0 \\
2.3 \\
2.5\end{array}$ & $\begin{array}{c}l b / y d^{3}{ }^{3} \\
677 \\
609 \\
572 \\
586 \\
630 \\
611\end{array}$ & $\begin{array}{l}7 \\
50 \\
52 \\
61 \\
49 \\
56 \\
52\end{array}$ & $\begin{array}{c}p s i \\
4970 \\
5310 \\
4200 \\
5640 \\
4240 \\
4780\end{array}$ & $\begin{array}{r}\% \\
0.052 \\
.076 \\
.049 \\
.059 \\
.054 \\
.065\end{array}$ & $\begin{array}{r}c \\
0.148 \\
.245 \\
.161 \\
.167 \\
.169 \\
.184\end{array}$ & $\begin{array}{c}\% \\
0.086 \\
.169 \\
.112 \\
.108 \\
.115 \\
.119\end{array}$ & $\begin{array}{c}\% \\
0.096 \\
.163 \\
.092 \\
.111 \\
.103 \\
.114\end{array}$ \\
\hline
\end{tabular}

1 Creep adjusted to a stress-strength ratio of 0.5 .

TABLE 10. Comparison of creep test data from moist-cured and steam-cured concretes made with same aggregates

\begin{tabular}{|c|c|c|c|c|c|c|c|c|c|c|}
\hline \multirow[b]{2}{*}{ Test } & \multirow[b]{2}{*}{ Aggregate } & \multirow{2}{*}{$\begin{array}{l}\text { Type of } \\
\text { Cement }\end{array}$} & \multirow[b]{2}{*}{ Cure } & \multirow{2}{*}{$\begin{array}{l}\text { Stress- } \\
\text { strength } \\
\text { ratio }\end{array}$} & \multirow{2}{*}{$\begin{array}{l}\text { Com- } \\
\text { pressive } \\
\text { strength } \\
\text { when } \\
\text { loaded }\end{array}$} & \multirow{2}{*}{$\begin{array}{l}\text { Slump of } \\
\text { concrete }\end{array}$} & \multicolumn{3}{|c|}{ Measured values at 1 year } & \multirow{2}{*}{$\begin{array}{c}\text { Ad- } \\
\text { justed } \\
\text { creep } 1 \\
\text { at } 1 \text { year }\end{array}$} \\
\hline & & & & & & & $\begin{array}{l}\text { Shrink- } \\
\text { age }\end{array}$ & $\begin{array}{l}\text { Creep } \\
\text { plus } \\
\text { shrink- } \\
\text { age }\end{array}$ & Creep & \\
\hline $\begin{array}{l}\text { II } \\
\text { Series } \mathbf{A} \\
\text { II } \\
\text { Series } \mathbf{A} \\
\text { II } \\
\text { Series A } \\
\text { Series A. }\end{array}$ & $\begin{array}{l}27 \\
27 \\
27 \\
30 \\
30 \\
\mathrm{E} \\
\mathrm{E} \\
\mathrm{ED}\end{array}$ & $\begin{array}{l}\text { I } \\
\text { IIII } \\
\text { I } \\
\text { IIII } \\
\text { I } \\
\text { IIII } \\
\text { III }\end{array}$ & 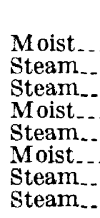 & $\begin{array}{l}\% \\
50 \\
56 \\
52 \\
63 \\
53 \\
58 \\
53 \\
56\end{array}$ & $\begin{array}{c}p s i \\
2390 \\
3590 \\
3820 \\
1890 \\
3770 \\
2070 \\
3780 \\
3550\end{array}$ & $\begin{array}{l}\text { in. } \\
2.0 \\
2.3 \\
2.5 \\
1.0 \\
2.8 \\
1.5 \\
2.3 \\
3.0\end{array}$ & $\begin{array}{c}\% \\
0.058 \\
.054 \\
.065 \\
.055 \\
.066 \\
.051 \\
.051 \\
.049\end{array}$ & $\begin{array}{c}\% \\
0.161 \\
.169 \\
.184 \\
.178 \\
.218 \\
.176 \\
.151 \\
.150\end{array}$ & $\begin{array}{l}\% \\
0.103 \\
.115 \\
.119 \\
.123 \\
.152 \\
.125 \\
.100 \\
.101\end{array}$ & $\begin{array}{c}\% \\
0.103 \\
.103 \\
.114 \\
.097 \\
.143 \\
.108 \\
.095 \\
.090\end{array}$ \\
\hline
\end{tabular}

I Creep adjusted to a stress-strength ratio of 0.5 .

TABLE 11. Effect of type of cure on creep, shrinkage and strength of a lightweight concrete made with aggregate 27

\begin{tabular}{|c|c|c|c|c|c|c|c|c|c|c|}
\hline \multicolumn{2}{|c|}{ Curing method } & \multicolumn{5}{|c|}{ Compressive strength } & \multicolumn{3}{|c|}{1 year measured values } & \multirow{2}{*}{$\begin{array}{c}\text { A djusted } \\
\text { creep } 1 \\
\text { at } 1 \\
\text { year }\end{array}$} \\
\hline Steam & $\begin{array}{l}\text { Moist } \\
\text { storage }\end{array}$ & 1 day & 3 day & 7 day & 28 day & 1 year & Shrinkage & $\begin{array}{c}\text { Crcep } \\
\text { plus } \\
\text { shrinkage }\end{array}$ & Creep & \\
\hline $\begin{array}{l}12 \mathrm{Hrs} . \\
12 \mathrm{Hrs} . \\
\text { None....... }\end{array}$ & $\begin{array}{l}\text { None....... } \\
7 \text { Days... } \\
7 \text { Days... }\end{array}$ & $\begin{array}{c}p s i \\
4,690 \\
3,180\end{array}$ & $\begin{array}{c}p s i \\
5,030 \\
4,700 \\
4,240\end{array}$ & $\begin{array}{c}p s i \\
5,220 \\
5,220 \\
5,030\end{array}$ & $\begin{array}{c}p s i \\
5,670 \\
5,630 \\
5,620\end{array}$ & $\begin{array}{c}p 8 s i \\
5,270 \\
5,700 \\
5,810\end{array}$ & $\begin{array}{c}\% \\
0.063 \\
.068 \\
.072\end{array}$ & $\begin{array}{c}\% \\
0.162 \\
.165 \\
.176\end{array}$ & $\begin{array}{c}\% \\
0.099 \\
.097 \\
.104\end{array}$ & $\begin{array}{l}\% \\
0.130 \\
.128 \\
.130\end{array}$ \\
\hline
\end{tabular}

${ }^{1}$ Creep adjusted to a stress-strength ratio of 0.5 .

TABLE 12. Effect of minus 200-mesh aggregate on creep and shrinkage

\begin{tabular}{|c|c|c|c|c|c|c|c|c|c|}
\hline \multirow[b]{2}{*}{ Test } & \multirow[b]{2}{*}{ Aggregate } & \multirow{2}{*}{$\begin{array}{l}\text { Cement } \\
\text { content }\end{array}$} & \multirow{2}{*}{$\begin{array}{c}\text { A mount } \\
\text { of - } 200 \\
\text { material } \\
\text { in fine } \\
\text { aggregate } \\
\text { by weight }\end{array}$} & \multirow{2}{*}{$\begin{array}{l}\text { Stress- } \\
\text { strength } \\
\text { ratio }\end{array}$} & \multicolumn{3}{|c|}{1 year measured values } & \multicolumn{2}{|c|}{$\begin{array}{l}1 \text { year adjusted } \\
\text { values } 1\end{array}$} \\
\hline & & & & & Shrinkage & Creep & $\begin{array}{c}\text { Creep } \\
\text { plus } \\
\text { shrinkage }\end{array}$ & Creep & $\begin{array}{c}\text { Creep } \\
\text { plus } \\
\text { shrinkage }\end{array}$ \\
\hline $\begin{array}{l}\text { Series } A \\
\text { IV } \\
\text { Series } A \\
\text { IV } \\
\text { Series } A \\
\text { IV }\end{array}$ & $\begin{array}{l}20 \\
27 \\
\mathbf{E}\end{array}$ & $\begin{array}{l}l b / y d^{3} \\
647 \\
684 \\
611 \\
658 \\
454 \\
490\end{array}$ & $\begin{array}{r}0 \% \\
11 \\
2 \\
3 \\
11 \\
2 \\
8\end{array}$ & $\begin{array}{l}\% \\
45 \\
37 \\
52 \\
43 \\
53 \\
41\end{array}$ & $\begin{array}{l}\% \\
0.084 \\
.071 \\
.065 \\
.066 \\
.051 \\
.043\end{array}$ & $\begin{array}{l}\% \\
0.135 \\
.114 \\
.119 \\
.100 \\
.100 \\
.063\end{array}$ & $\begin{array}{c}\% \\
0.219 \\
.186 \\
.184 \\
.166 \\
.151 \\
.106\end{array}$ & $\begin{array}{r}\% \\
0.149 \\
.154 \\
.114 \\
.117 \\
.095 \\
.077\end{array}$ & $\begin{array}{l}\% \\
0.233 \\
.225 \\
.179 \\
.183 \\
.146 \\
.120\end{array}$ \\
\hline
\end{tabular}

Creep adjusted to a stress-strength ratio of 0.5 . 
made with normal-weight aggregate $E$ indicate that the slump did not appreciably affect the creep and shrinkage.

Data from the concretes from test I of series B, which were obtained in creep tests at three stress levels, are combined and analyzed with similar data from test II. In making the adjustments to measured creep values throughout this paper it was assumed that over the usually narrow ranges over which the adjustments were made the creep of a concrete was proportional to the ratio of the applied stress to the concrete strength at the time of loading. Test I and II were set up so that this assumption could be tested.

Creep data for the various stress-streng th ratios used in tests I and II are plotted in figure 13. In drawing the curves shown on figures 13 and 14 (which was derived from fig. 13) it was assumed that the origin was a point on each curve. The curve shown in figure 14 represents the average of the five curves of figure 13, but with the creep values expressed as percentages of the average creep at a stress-strength ratio of 50 percent.

It can be seen that the assumption made previously that the creep is proportional to the stressstrength ratio is not strictly true. Since the error from making the adjustments does not appear to have been great, all adjustments made to creep values hereafter in this report are also based on the assumed straight line proportionality.

Upon studying figure 13 it will be noticed that although different cures, aggregates and strengths, as well as cements, are involved, the spread in the creep values at any particular stress-strength ratio is not great. To show more clearly the effect of the stress-strength ratio on the creep

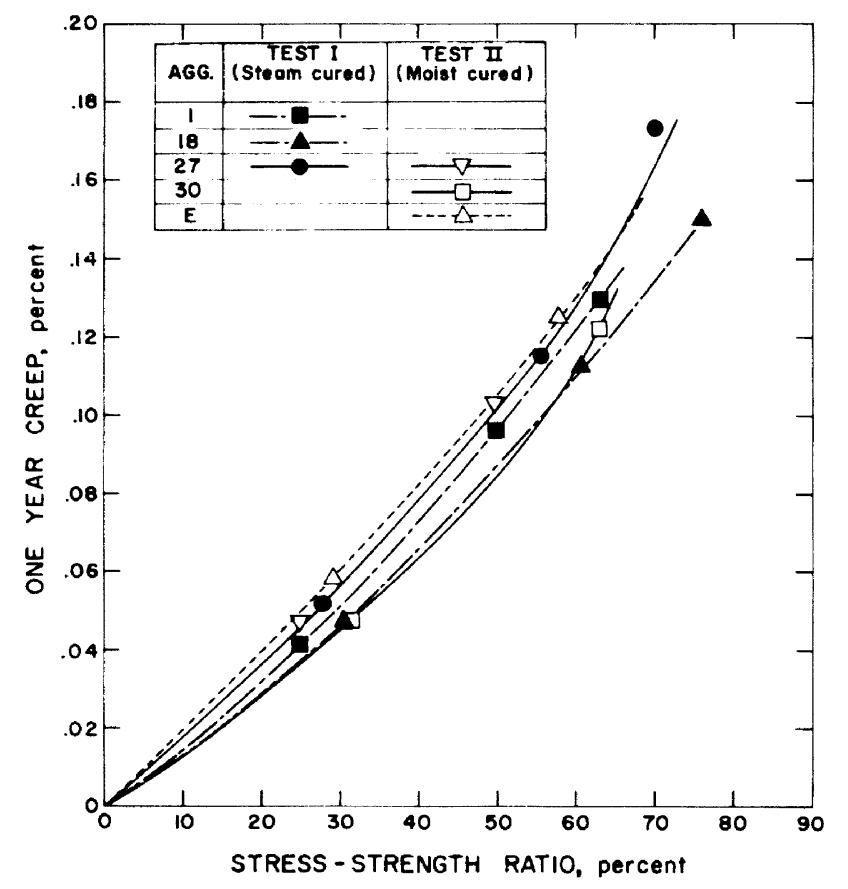

Figure 13. Relationship between the creep and the stressstrength ratio for some series B concretes.

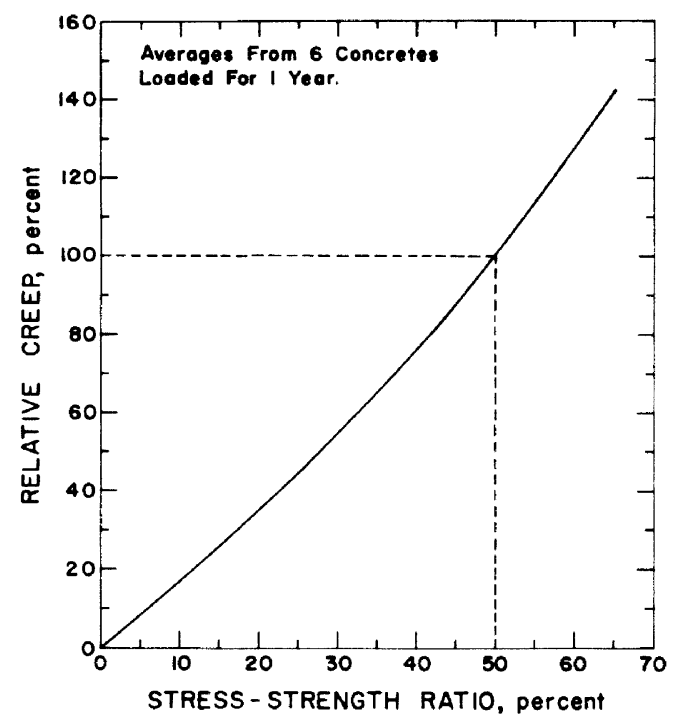

FIGURE 14. Relationship between relative creep and the stress-strength ratio for some series B concretes.

The curve represents the average from the curves presented as figure $\mathbf{1 3 .}$

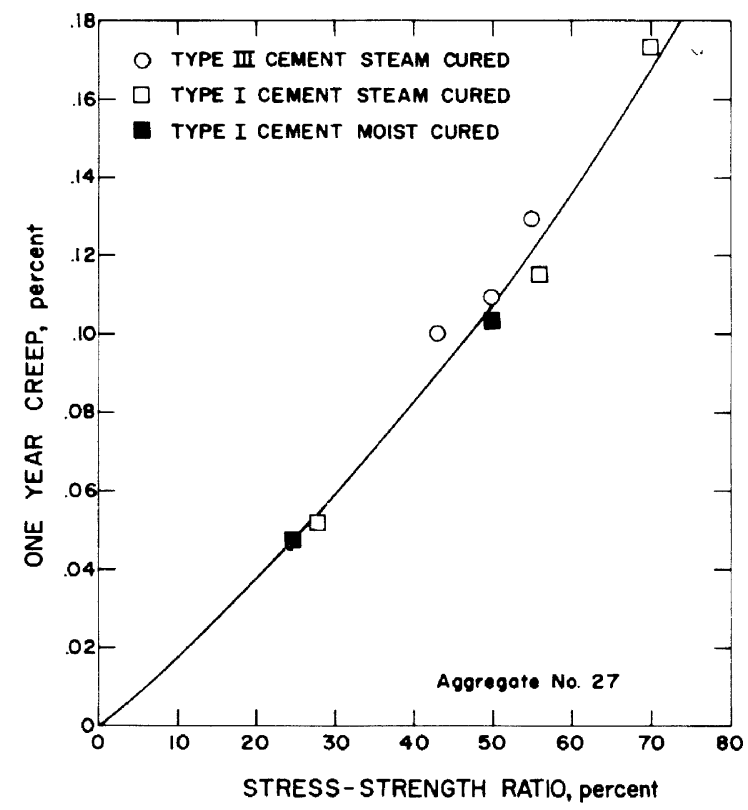

FIGURE 15. Relationship between the creep and the stressstrength ratio for concretes made from agregate 27.

of concrete, all available creep data for concretes made with aggregate 27 are plotted in figure 15 . The correlation between creep and stress-strength ratio appears rather remarkable when it is considered that three cures, two types of cement, and two nominal strength- and cement-content ranges are represented in this figure.

\subsubsection{Supplemental Test III}

Test III was designed to investigate whether creep and shrinkage of steam-cured concretes are less than for comparable moist-cured concretes, as reported by Shideler [2].

A single batch of concrete was made with aggregate 27 and type III cement. Two-thirds of the 36 test cylinders cast were steam cured. The 
balance of the cylinders and half of the steamcured cylinders were moist cured until 7 days old. At this age, when all the specimens had nearly the same compressive strength, one cylinder representing each of the three cures was placed under 2000 psi load in a single creep test frame. Pertinent information on the test III specimens and data from test III are given in tables 8 and 11 . It can be seen that after 1 year the steam-cured specimens have exhibited slightly less creep and shrinkage than the specimens with only the moist cure. Shideler [2] reported that steam-cured concretes exhibited as much as 50 percent less creep than moist-cured concretes. However, by adjusting his data for the stress-strength ratio this apparent difference in creep is decreased to less than 25 percent.

As the reduction in creep and shrinkage of the steam-cured compared with the moist-cured concrete does not appear to be as significant in these tests as reported elsewhere, it is thought that differences in the reactions of different cements to the steam cure may account for the difference in the behavior of the concretes in the different in vestigations.

\subsubsection{Supplemental Test IV}

Test IV was designed to explore more fully the indications of the early results from the series $A$ test, which appeared to indicate that concretes made with aggregates containing larger amounts of material passing the 200 sieve exhibited greater creep and shrinkage. It was reasoned that if the amount of this fine material were changed significantly, a significant change should occur in the creep and shrinkage.

The concretes for test IV were designed, cured, and tested the same as series A concretes, except for the amount of material passing 200 mesh sieve which was introduced as a variable. The pertinent data obtained in this test are given in table 12. These data would seem to indicate that there is no significant or consistent effect on the creep and shrinkage due to the changes in the quantities of the minus 200 material used in this investigation.

One explanation of this apparent lack of agreement between the eurlier results and the subsequent tests is the possibility that the softer aggregates, which might be expected to exhibit greater creep and shrinkage, might also contain a greater quantity of very fine material because of breakdown of the aggregate with handling.

At the present time there is no standard test method for rating the hardness of these aggregates, so that any comparison may be questionable.

\subsubsection{Supplemental Test $\mathrm{V}$}

Test $V$ was designed to determine the effect of replacement of some lightweight aggregate by a normal-weight aggregate on creep and shrinkage. It is the practice in some parts of the country to use natural sand to replace lightweight fines when making lightweight concretes. The sand is used in some cases to increase the modulus of elasticity of the concrete and in other cases is used for reasons of economy.

TABLE 13. Effect of partial replacement of lightweight with normal-weight aggregate

\begin{tabular}{|c|c|c|c|c|c|c|c|c|c|c|c|c|}
\hline \multirow{2}{*}{ Test } & \multicolumn{2}{|c|}{ Aggregate used } & \multirow{2}{*}{$\begin{array}{l}\text { Stress- } \\
\text { strength } \\
\text { ratio }\end{array}$} & \multirow{2}{*}{$\begin{array}{l}28 \text {-day } \\
\text { strength }\end{array}$} & \multirow{2}{*}{$\begin{array}{l}28 \text {-day } \\
\text { secant } \\
\text { modulus }\end{array}$} & \multirow{2}{*}{$\begin{array}{c}28-d a y \\
\text { unit } \\
\text { weight }\end{array}$} & \multirow{2}{*}{$\begin{array}{c}\text { Ovendry } \\
\text { unit } \\
\text { weight }\end{array}$} & \multicolumn{3}{|c|}{ Measured values at 1 year } & \multicolumn{2}{|c|}{$\begin{array}{l}1 \text { year adjusted } \\
\text { values } 1\end{array}$} \\
\hline & Fine & Coarse & & & & & & Shrinkage & $\begin{array}{c}\text { Creep } \\
\text { and } \\
\text { shrinkage }\end{array}$ & Creep & Creep & $\begin{array}{c}\text { Creep } \\
\text { and } \\
\text { shrinkage }\end{array}$ \\
\hline $\begin{array}{l}\text { VI } \\
\text { VI } \\
\text { Series A } A \\
\text { Series A... }\end{array}$ & $\begin{array}{l}\text { Sand } \\
25 \ldots \\
\text { Sand } \\
25 \\
14\end{array}$ & $\begin{array}{l}25 \\
\text { Gravel } \\
14 \\
25 \\
14\end{array}$ & $\begin{array}{l}\% \\
54 \\
48 \\
58 \\
48 \\
38\end{array}$ & $\begin{array}{c}p s i \\
5240 \\
5750 \\
4960 \\
6640 \\
6430\end{array}$ & $\begin{array}{c}10^{6} \mathrm{psi} \\
2.94 \\
3.26 \\
2.44 \\
2.42 \\
1.71\end{array}$ & $\begin{array}{c}p c f \\
114 . \\
121 . \\
107 . \\
101 . \\
90 .\end{array}$ & $\begin{array}{c}p c f \\
109 . \\
116 . \\
102 . \\
95 . \\
78 .\end{array}$ & $\begin{array}{c}\% \\
0.056 \\
.051 \\
.063 \\
.061 \\
.073\end{array}$ & $\begin{array}{c}\% / \\
0.212 \\
.173 \\
.240 \\
.191 \\
.198\end{array}$ & $\begin{array}{c}\% \\
0.156 \\
.122 \\
.177 \\
.130 \\
.125\end{array}$ & $\begin{array}{c}\% \\
0.145 \\
.127 \\
.153 \\
.137 \\
.167\end{array}$ & $\begin{array}{c}\% \\
0.201 \\
.178 \\
.216 \\
.198 \\
.240\end{array}$ \\
\hline
\end{tabular}

1 Creep adjusted to a stress-strength ratio of 0.5 .

$\mathrm{T}_{\mathrm{ABLE}} 14$. Effect of varying the moisture content of aggregates at the time of mixing on creep and shrinkage

\begin{tabular}{|c|c|c|c|c|c|c|c|c|c|c|c|c|c|}
\hline \multirow{2}{*}{ Test } & \multirow{2}{*}{ Aggregate } & \multicolumn{2}{|c|}{$\begin{array}{l}\text { Aggregate mois- } \\
\text { ture content } \\
\text { when batched } \\
\text { (by weight) }\end{array}$} & \multicolumn{3}{|c|}{ Unit weight } & \multirow{2}{*}{$\begin{array}{l}\text { Stress- } \\
\text { strength } \\
\text { ratio }\end{array}$} & \multirow{2}{*}{$\begin{array}{l}\text { 28-day } \\
\text { strength }\end{array}$} & \multicolumn{3}{|c|}{ Measured values at 1 year } & \multicolumn{2}{|c|}{$\begin{array}{c}\text { Adjusted values at } \\
1 \text { year }{ }^{2}\end{array}$} \\
\hline & & Fine & Coarse & 1 day & $\begin{array}{l}\text { 28-day } \\
\text { air dry }\end{array}$ & $\begin{array}{l}\text { Oven } \\
\text { dry }\end{array}$ & & & $\underset{\text { age }}{\text { Shrink- }}$ & $\begin{array}{l}\text { Creep } \\
\text { plus } \\
\text { shrink- } \\
\text { age }\end{array}$ & Creep & Creep & $\begin{array}{l}\text { Creep } \\
\text { plus } \\
\text { shrink- } \\
\text { age }\end{array}$ \\
\hline $\begin{array}{l}\text { VI } \\
\text { Series A } \\
\text { VI } \\
\text { VI... } \\
\text { Series A }\end{array}$ & $\begin{array}{l}4 \\
41 \\
14 \mathrm{D} \\
14 \mathrm{~W} \\
14{ }_{1}{ }^{2}\end{array}$ & $\begin{array}{r}\% \\
2.0 \\
14.5 \\
2.4 \\
29.9 \\
6.8\end{array}$ & $\begin{array}{l}\% \\
0.2 \\
1.5 \\
0 \\
27.0 \\
9.5\end{array}$ & $\begin{array}{r}p c f \\
99 \\
101 \\
91 \\
96 \\
97\end{array}$ & $\begin{array}{l}p c f \\
94 \\
96 \\
85 \\
86 \\
90\end{array}$ & $\begin{array}{l}p c f \\
88 \\
89 \\
76 \\
76 \\
78\end{array}$ & $\begin{array}{l}07 \\
43 \\
50 \\
37 \\
43 \\
38\end{array}$ & $\begin{array}{c}p s i \\
6070 \\
5570 \\
6720 \\
6120 \\
6430\end{array}$ & $\begin{array}{c}\% \\
0.073 \\
.076 \\
.077 \\
.085 \\
.073\end{array}$ & $\begin{array}{r}\% \\
0.200 \\
.221 \\
.199 \\
.238 \\
.198\end{array}$ & $\begin{array}{l}\% \\
0.127 \\
.145 \\
.122 \\
.153 \\
.125\end{array}$ & $\begin{array}{l}\% \% \\
0.147 \\
.146 \\
.165 \\
.177 \\
.167\end{array}$ & $\begin{array}{r}\% \\
0.220 \\
.222 \\
.242 \\
.262 \\
.240\end{array}$ \\
\hline
\end{tabular}

1 As received.

2 Creep adjusted to a stress-strength ratio of 0.5 
Three batches of concrete were made with the type III cement and two lightweight aggregates. One batch (designated $25 \mathrm{~S}$ ) was made by combining the local natural sand with the coarse fraction of aggregate 25. The second batch (designated $25 \mathrm{G}$ ) was made using the lightweight fines of aggregate 25 with the $3 / 4$-in. maximum size $W M$ gravel. The third batch was made with the local sand and the coarse fraction of aggregate 14 . The mixes were designed to have approximately the same strength as the series A concretes. The cure and test procedure were also the same in series A.

From an analysis of the data presented in table 13 it appears that the practice of replacing lightweight aggregate fines with natural sand may reduce the drying shrinkage. The replacement of lizhtweight fines by natural sand reduced the drying shrinkage for aggregates 14 and 25 by 14 and 8 percent, respectively; the substitution of WM gravel for the coarse fraction of aggregate 25 resulted in a reduction of shrinkage of 16 percent. However, the adjusted values of creep at 1 year were not consistently reduced. The substitution of the sund for the lightweight fines resulted in a slight increase in the creep for concrete made with aggregate 14 , but a decrease for aggregate 25 concrete. The substitution of gravel for the aggregate 25 coarse fraction resulted in a slight reduction in creep.

\subsubsection{Supplemental Test VI}

The effect of varying the amounts of moisture in the aggregates at the time of mixing on the creep and shrinkage of lightweight concretes was investigated in test VI.

In the series $A$ tests most of the aggregates were batched when relatively dry (see table 3) except for the fines of aggregate 4 which had been shipped and stored in plastic-lined bags. The first of three concretes for test VII was made with the aggregate 4 after it had been dried. The second and third concretes were made with air-dry and very moist aggregate 14 respectively. Table 14 gives pertinent data for test VI and comparable data from series A concretes.

It appears from the data in table 14 that the practice of soaking lightweight aggregates before batching probably causes only a slight increase in creep and shrinkage of the concrete over that exhibited by concretes made with dry-batched aggregates. It should be remembered that although the moisture condition of the aggregates varied considerably when batched, the $10-\mathrm{min}$ waiting or soaking period used in mixing the concretes eliminated much of the apparent difference between the dry- and wet-batched aggregates.

The absorption characteristics of the lightweight aggregates vary considerably, but approximately 50 percent of the $24-\mathrm{hr}$ absorption occurs in the first $10 \mathrm{~min}$ that free water is available. The absorption after the first $10 \mathrm{~min}$, although at a relatively slow rate contributes to a loss of slump after mixing. This absorption of water by the aggregate after mixing is usually called "watergrab".

\section{Discussion of Results}

It is the usual practice to compare lightweight with "regular" normal-weight concrete as if the "regular" concrete had properties which are standard regardless of the agrgregates used. However, the data obtained in this study and by other investigators indicate that there is a considerable spread in the properties of concretes of the same nominal strength made either with natural, normal-weight aggregates or with manufactured, lightweight aggregates. In fact, there is such an overlapping of the data from the two types of concrete that it would seem to indicate that except for flexural strength the mechanical properties of some lightweight concretes are equivalent to the properties of some normal-weight concretes.

\subsection{Significance of Slump as a Measure of Water Content of Concrete}

With normal-weight concretes made with lowabsorption normal-weight aggregates the measured slump can be regarded as a fair index of the relative water-cement ratio, but with the lightweight concretes some judgment must be used in comparing the concretes on the basis of the reported values of slump. The lightweight mixes were proportioned on a cement content-slump basis because for most lightweight aggregates the absorption characteristics are such that the net water-cement ratio cannot be determined accurately. Moreover, these same absorption characteristics can also introduce errors in determining the slump except when the aggregates are saturated or nearly so. Consequently when we consider the possible effect of slump on creep as we did in sec. 7.2.1, we must also consider whether mixing conditions were identical, since the slump of lightweight concrete reflects both the net watercement ratio as well as the "water-grab" of the aggregutes.

As the rate of absorption is relatively high during the early part of the period when free moisture is a railable to the aggregate, a difference of a few minutes can appreciably affect the mixing water necessary for a given slump. The "watergrab" was very noticeable when mixing many of the air-dry lightweight aggregates, even with the $10 \mathrm{~min}$ soaking period used in this investigation.

All values of slump reported here are for concretes as discharged from the mixer and do not necessarily indicate the consistency as placed since the "water-grab" results in a loss of slump.

\subsection{Statistical Analysis of Test Results}

It is impractical to tabulate all the data from the individual tests performed in this investigation. 
TABLE 15. Statistical analyses of test results

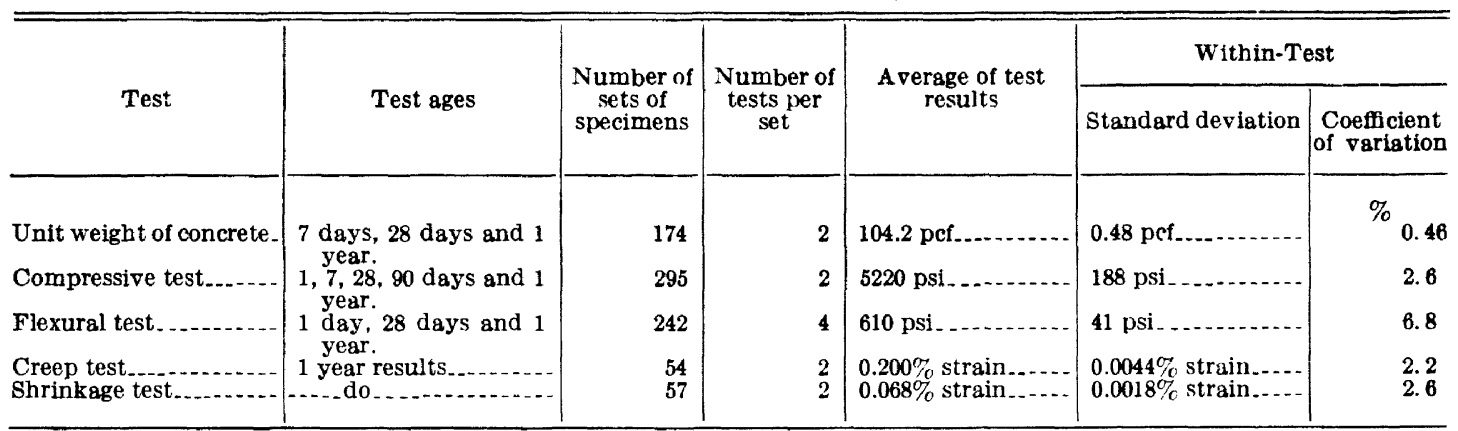

However, as an aid in determining the significance of apparent differences in test values between different concretes, within-test values of the standard deviations and coefficients of variation were determined by the method recommended in [13]. These values, which are given in table 15 , are a convenient method of expressing the efficiency of the test procedures. However, for these test results the within-test factors, with the exception of those for unit weight also include the variability of the concretes. The variation in the unit weights of duplicate specimens of the same batch of concretes allows some insight into the variability of the concretes and is included in table 15 for this reason. A spread of one standard deviation in the unit weights between 2 lightweight concretes made from the same aggregate, corresponds to a spread in the 28 day compressive strength of about 200 psi.

\subsection{Compressive Strength}

As was mentioned in sec. 7.1.1 the most significant finding from the compressive-strength data for the series A concrete was the relatively small gain in strength from 28 to 90 days and the slight drop in strength after 90 days. 'This finding for steam-cured concretes may appear to be opposite to the usual finding for moist-cured concretes. However, it has been reported by Price [14] and others, that concretes moist-cured for a limited time and then air dried will exhibit a similar retrogression in compressive strength. This would seem to indicate that the retrogression in the strength of the steam-cured concretes was not so much the result of the steam cure, per se, as it was a result of the interruption in the moisture supply.

Hanson [15] and Higginson [16] have shown that even 28-day compressive strengths are lower for steam-cured than for continously moist-cured concretes. When it is remembered that steamcured specimens are usually relatively dry when tested and that the continuously moist-cured specimens are tested damp this difference acquires added significance.

It seems that the compressive strength-time curves for steam-cured and limited moist-cured concretes are similar in shape, but the st eam-cure strength level is below the moist-cure at 28 days and older.
The actual gain in strength from 1 day to 28 days raried considerably for the series $A$ concretes. It appears that the gain in strength is probably dependent on some characteristic of the aggregate as well as on the type of cure and cement. It is possible that the small rate of gain in strength of concretes for some of the agrogregates reflects the fact that the concretes are appraoching the maximum strengths which can be obtained with those aggregates.

\subsection{Modulus of Elasticity}

Many attempts have been made to devise an empirical formula with which values for the modulus of elasticity of concrete can be computed from strength and unit-weight data with reasonable accuracy. The most recent formula proposed [17] is

where

$$
E=33 W^{\top / 2} \sqrt{f_{c}^{\prime}}
$$

$E=$ static modulus of elasticity, psi

$W=$ unit weight of the concrete at the time of test, pef

$f^{\prime}{ }_{c}=$ compressive strength of concrete at the time of test, psi.

The data from this investigation were plotted in figure 16 to show the relationship between the secant modulus and $W^{3 / 2} \sqrt{f^{\prime}}$. As can be seen from figure 16 there is considerable scatter from the proposed line. However, if the data from the cranite concretes, indicated in this plot by the letter $G$, are excluded the correlation is better.

As would be expected, an even better correlation is shown in figure 17 where the plotted data is for concretes made with but one acroregate-No. 27 .

For purpose of comparison figure 18 presents some data for insulating concretes from a previous unpublished NBS investigation. The proposed formula is shown to be reliable for the low-density and low-strength concretes also.

\subsection{Flexural Strength}

Walker and Bloem [7] found that partially dried conrretes have lower flexural strength than continuously moist-cured concretes. They also found indications that, other factors being comparable, thoroughly dry concrete produced higher flexural strength than did moist concrete. 


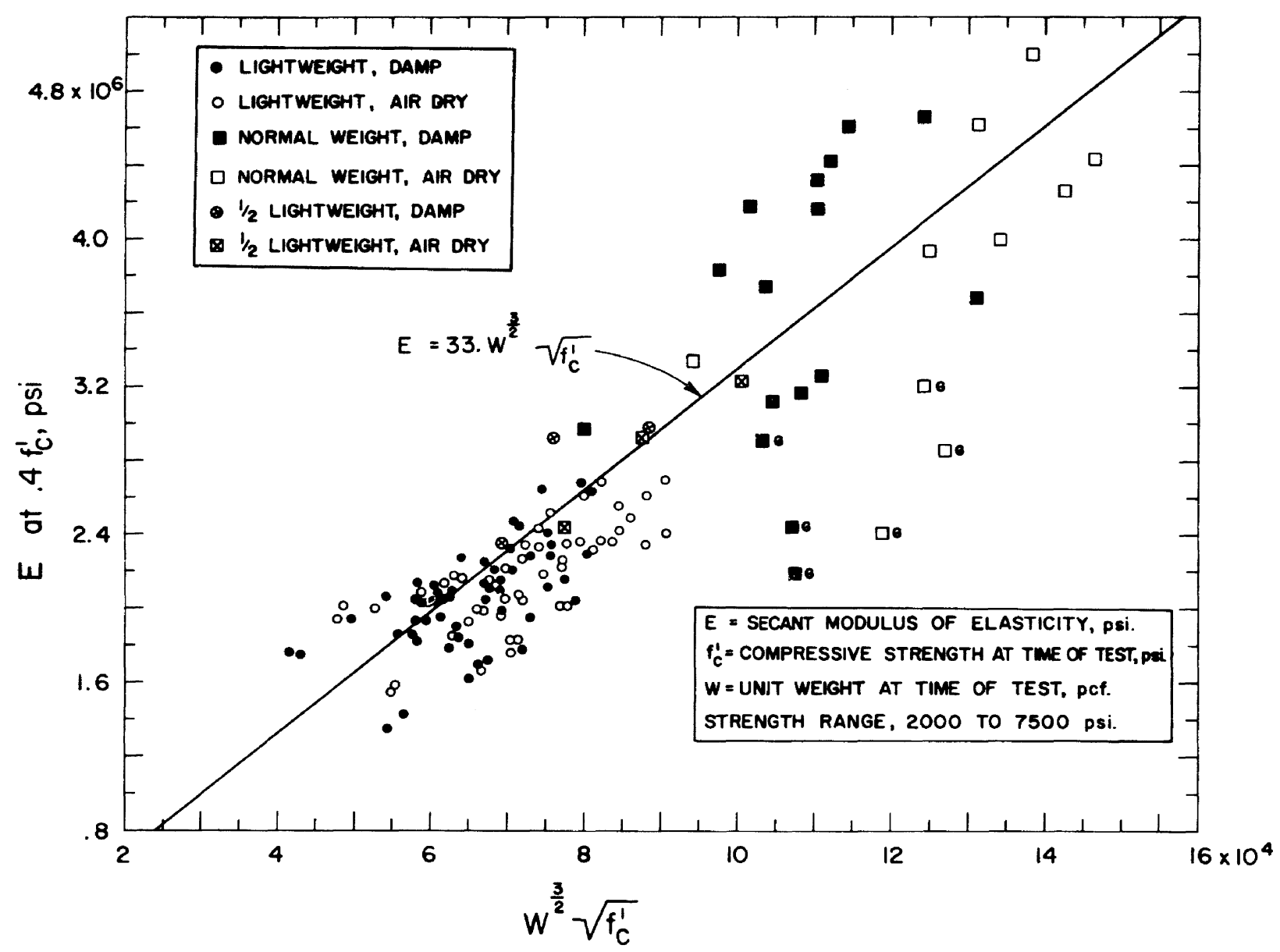

FIgURe 16. Relationship between the secant modulus of elasticity and the compressive strength and unit weight of concretes. The secant moduli were those obtained for $0.4 \mathbf{f}^{\prime}{ }_{c}$.

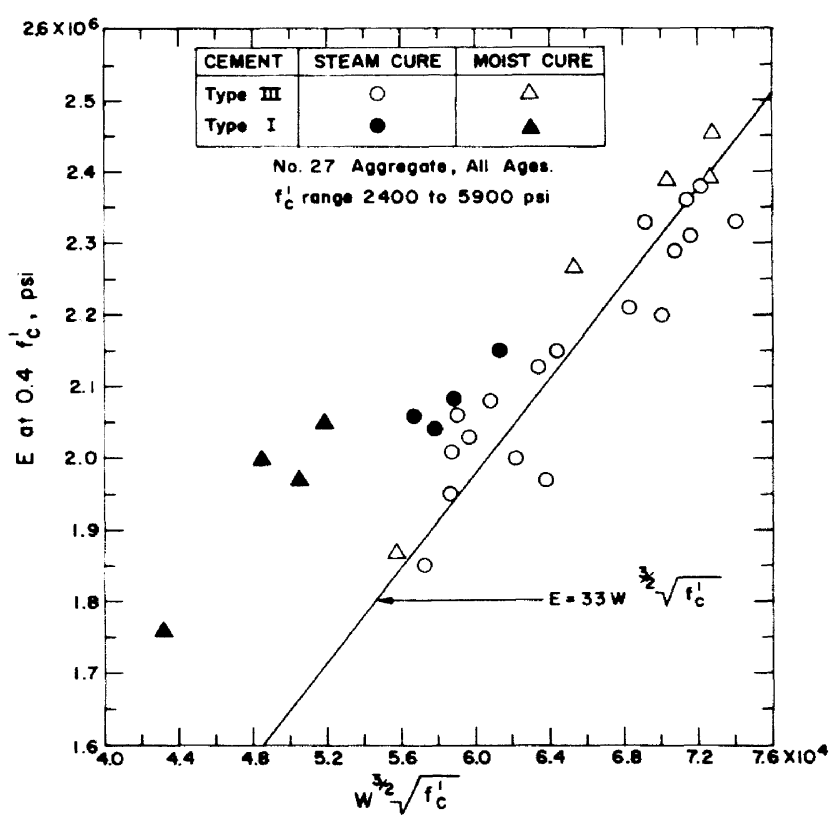

Figure 17. Relationship between the secant value of the modulus of elasticity (E) and the compressive strength $\left(\mathrm{f}^{\prime}{ }_{\mathrm{c}}\right)$ and unit weight $(\mathrm{W})$ of concretes made. with aggregate $2 \%$.

Plotted values of $\mathrm{E}$ were at 0.4 the compressive strength. $\mathrm{t}^{\prime} \mathrm{c}$ varied from 2400 to $5900 \mathrm{psi}$. W varied from 84 to about 102 pef.
The results from this investigation indicate that for concretes made with certain dense aggregates the flexural strength of the partially dried concrete may be higher than for the moist concrete. However, the partially dried lightweight concretes always had a lower flexural strength than the moist concretes. No correlation could be found between the wet-dry flexural strength ratio and the drying shrinkage of the concrete as measured on the 6- by 12-in. cylinders. However, the effect of the partial drying on the flexural strength appears to be related to some as yet unidentified property of the concrete aggregate.

In a supplementary test, not presented in this report, it was found that 1-hour air drying reduced the flexural strength of specimens made from lightweight concretes about the same as 27-day air drying.

\subsection{Creep and Shrinkage}

It has been noticed by many observers that the shrinkage of concrete members in the field is sometimes much less than for similar laboratory specimens even when average humidity and temperature conditions are the same and the members are protected from rain. This phenomenon is generally explained by the fact that concrete 


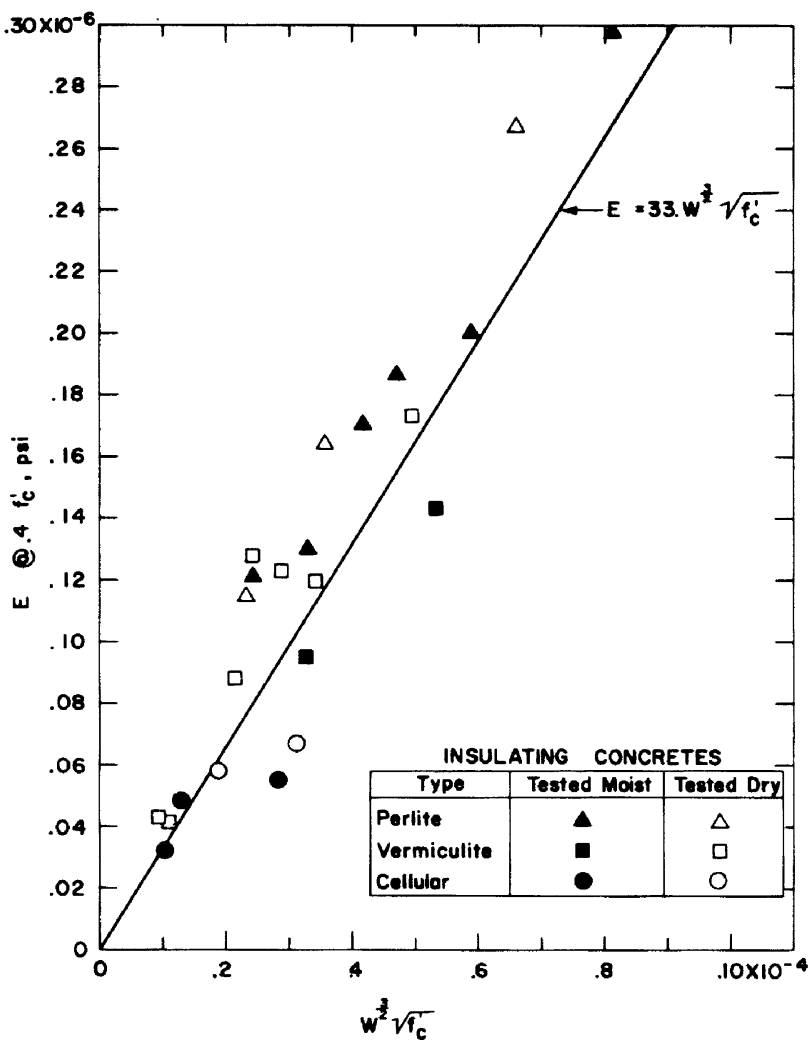

FIGURE 18. Relationship between the secant modulus of elasticity $(\mathrm{E})$ and the compressive strength $\left(\mathrm{f}^{\prime}{ }_{c}\right)$ and unit weight (W) of very lightweight concretes.

$\mathrm{f}^{\prime}{ }_{c}$ varied from 75 to $600 \mathrm{psi}$. W varied from about 23 to 48 pef.

will take up moisture much faster than it will release it. Under field conditions of alternate high and low humidities the concrete behaves as if it were exposed to much higher than the average ambient humidity.

In applying shrinkage values from a laboratory investigation to actual structures, exposure conditions should be considered. It is thought that the 1-year values reported here are probably close to the maximum which would be encountered over most of the United States for similar concretes. However, in certain areas, such as the Southwest, a combination of high ambient temperatures and low humidities would probably result in shrinkage values greater than those reported here.

The creep and shrinkage data presented here indicate that the creeps are roughly proportional to the drying shrinkages.

At the present time, long-term testing appears to be the only reliable method which can be used to judge the effect an aggregate will have on the creep and shrinkage of concrete. The data shown in figures 6 and 7 show that it might be possible to obtain a reliable estimate of long-term creep from a relatively short-term test, but the estimate is considerably less reliable for shrinkage.

In interpreting the creep data it should be remembered that the specimens tested for this investigation were kept loaded at a constant stress level. In a sense this test method is directly applicable only to structural members subjected to a constant sustained stress uniform over the cross section of the member; it is somewhat unrealistic in its application to prestressed concrete since the stress in a prestressed member is not uniform and is reduced in proportion to the length change of the concrete and with relaxation of the steel tendons. It has been shown [18] that a considerable loss in stress can occur due to relaxation of the steel. Three methods which can be used in determining prestress losses, when creep, shrinkage, and relaxation data are available, have been discussed in [18, 19, 20, and 21].

From the compressive-creep data presented here it is obvious that creep, as it was defined for this report, is not proportional to the applied load. Among other factors, this lack of proportionality can be explained if we assume that the drying shrinkage of a concrete is increased by an applied load. Neville [9] made some creep determinations on mortar specimens which were air dried before being placed under creep load and concluded that creep is proportional to the applied load when no drying shrinkage can occur.

The data presented in figures 13 and 15 show a relationship which is very significant in a structural design. These data indicate that one of the most significant factors affecting the creep of concrete is the stress-strength ratio. The effect of the stress-strength ratio on the creep overshadows the effect of most other factors. Variables such as type of cement, curing conditions, and aggregate gradation are factors affecting creep mainly insofar as they affect the stress-strength ratio.

The aggregate used in making the concrete appears to be another significant factor in creep. From the data presented in table 13 for aggregate 25 there is an indication that the creep and shrinkage are affected to a greater extent by the properties of the coarse particles than by those of the fines of the aggregate.

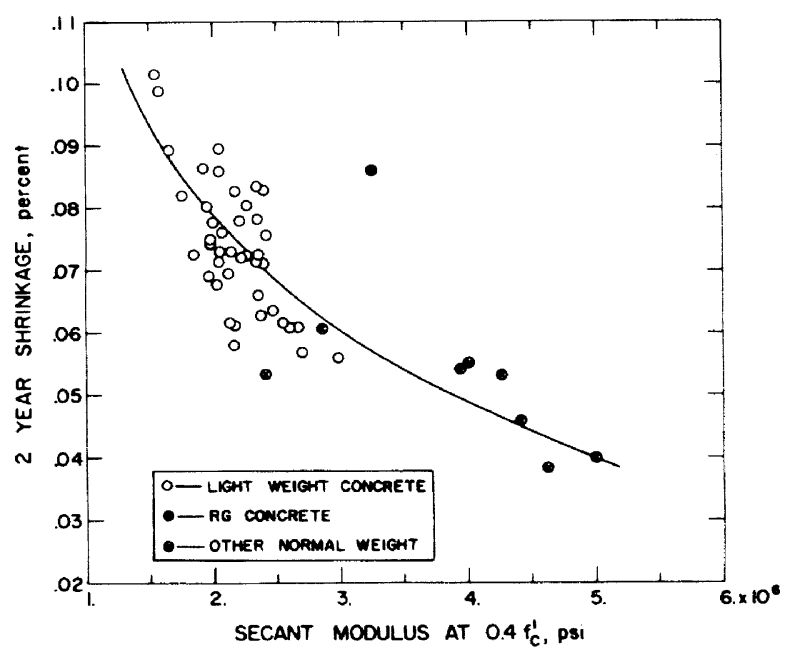

FIGURE 19. Relationship between the drying shrinkage and the secant modulus of elasticity of concrete. The modulus indicated is for the 28 day test. 
Carlson [22] concluded that the relative compressibility of the aggregate particles appeared to be the most important factor causing different aggregates to produce concretes of different shrinkage. The validity of this ussumption was investigated in this study by examining the relationship between the moduli of elasticity and the drying shrinkage of the concretes. Data from this investigation which are plotted in figure 19, indicate that such a correlation probably does exist between the shrinkage and modulus of elasticity. However, the creep data indicate poor or no correlation between the creep and the modulus of elasticity of the concrete.

\subsection{Conclusions}

1. Some lightweight concretes have practically the same mechanical properties as some normalweight concretes, except for flexural strength.

2 . It appears that the use of a steam-curing method which increases the early strength of a concrete is detrimental to the long-term strength gain.

3. If data from granite-aggregate concretes are excluded, the proposed empirical formula for the modulus of elasticity, $E=33 W^{3} / \sqrt[2]{f^{\prime}}{ }_{c}$, will yield values for both lightweight and normal-weight concretes reasonably close to observed values.

4. Partially dried lightweight concretes have lower flexural strength than continuously moist concretes. There are indications that other factors being comparable, the fully dried concretes may be equal in flexural strength to the moist concretes.
5. Partially dried concretes made from aggregate $E$, the only normal-weight concrete tested in flexure, had higher flexural strength than the moist concretes.

6. A concrete which exhibits relatively low values of creep will exhibit low values of drying shrinkage.

7. Two-year creep values may be estimated with good accuracy from 90-day creep values. The same is not true for the shrinkage values.

8. Creep as defined for this report is not strictly proportional to the stress-strength ratio, even at relatively low loads.

9. The stress-strength ratio appears to be one of the two significant factors affecting the creep of concrete. The effect of this ratio on creep overshadows the effect of many other variables, when exposure conditions are constant.

10. The type of aggregate used in making the concrete is the other significant factor in the creep of concrete. The property of the aggregate which determines the creep is as yet unidentified. It appears that the coarse aggregate is more effective than the fines in affecting the creep.

11. There appears to be a correlation between the shrinkage and the modulus of elasticity of the concrete. This correlation would be expected since the modulus of the concrete is an index of the compressibility of the aggregate used.

12. There was no evidence of any disruptive expansion reaction between any aggregate tested and the cement, or even any significant long-term moisture expansion of the aggregate itself.

\section{Appendix}

\subsection{Lightweight Aggregates}

The lightweight aggregates used in this investigation were either expanded shale, clay, or slate. However, neither an examination of the acoregates, nor an analysis of the performance of the aggregates in concrete appeared to furnish a means of identifying the raw material in the aggregates.

This type of lightweight agroregate is produced in rotary kilns, generally ranging in diameter from 8 to 12 feet and in length from 80 to 200 feet. The typical production process consists of introducing raw materials, grading from approximately one inch to the No. 50 screen, in to the kiln where the temperature is raised until it reaches a pyroplastic state at which time gases are generated and bloat the agcregate particles. The material is quickly discharged from the kiln and cooled. The bloating temperatures range from 1800 to 2100 ${ }^{\circ} \mathrm{F}$, depending upon the raw material. After cooling, the material is screened and oversize particles are crushed to fill out the gradation of both fine and coarse aggregate.

There are slight variations in the process, for example, in some cases raw material is separated into different size fractions which are expanded separately. Sometimes the kiln feed is presized to such an extent that no crushing is required after burning.
Examples of the agrogregates are shown in figure 20. The sample designated $A$ has a "coated" appearance. One of these particles has been sliced to show the cellular interior. Sample $B$ illustrates aggregate particles that have been crushed after processing so that the cellular interior structure is exposed. Table 16 gives a brief physical description of each lightweight aggregate used in this investigation.

\subsection{Strain Measurements}

The gage used for determining the changes in length of the creep, shrinkage, and moisture expansion specimens was a 10-in. Whittemore strain gage. This mechanical gage required two metal inserts spaced 10-in. apart in the concrete. The two pointed legs of the gage are seated into small holes in the inserts when making measurements.

Accuracy of the Whittemore is determined by the accuracy of the dial indicator. The sensitivity of the gage is about $\pm 2 \times 10^{-6}$ in./in., but practical considerations such as the precision of the gage holes in the inserts, consistency of measuring technique, and accuracy of temperature compensation increases the uncertainty of the measurements to an estimated $\pm 10 \times 10^{-6} \mathrm{in}$./in. 

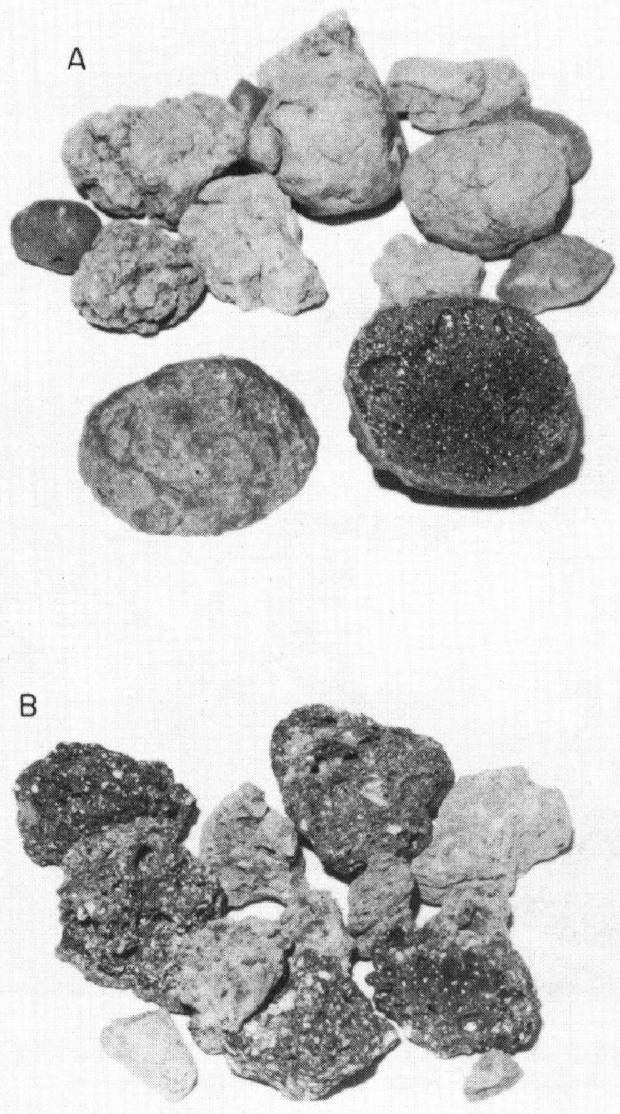

FIgURE 20. Two types of expanded-shale aggregate. A indicates typical coated particles with one particle cut open to show the cellular interior. $\mathrm{B}$ indicates typical crushed particles.

TABLE 16. Physical appearance of aggregate particles

\begin{tabular}{|c|c|c|c|}
\hline $\begin{array}{c}\text { Aggre- } \\
\text { gate }\end{array}$ & Color & Shape 1 & Surface texture ${ }^{2}$ \\
\hline & Medium gray & \multirow{9}{*}{ 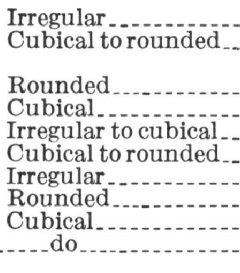 } & \multirow{23}{*}{$\begin{array}{l}\text { Smooth. } \\
\text { Mostly smooth. } \\
\text { Smooth. } \\
\text { Smooth to rough. } \\
\text { Do. } \\
\text { Do. } \\
\text { Smooth. } \\
\text { Do. } \\
\text { Rough. } \\
\text { Do. } \\
\text { Do. } \\
\text { Mostly smooth. } \\
\text { Do. } \\
\text { Do. } \\
\text { Smooth. } \\
\text { Mostly rough. } \\
\text { Rough. } \\
\text { Smooth. } \\
\text { Mostly smooth. } \\
\text { Rough. } \\
\text { Do. } \\
\text { Mostly smooth. } \\
\text { Smooth. } \\
\text { Rough. }\end{array}$} \\
\hline 2 & $\begin{array}{l}\text { Medium gray with } \\
\text { some brown. }\end{array}$ & & \\
\hline & Reddish brown & & \\
\hline 5 & Light gray & & \\
\hline & Dark gray & & \\
\hline 8 & Mixed gray and brown & & \\
\hline & $\begin{array}{l}\text { Grayish brown } \\
\text { Reddish brown }\end{array}$ & & \\
\hline 11 & Medium gray & & \\
\hline 14 & $\begin{array}{l}\text { Medium gray some } \\
\text { tans. }\end{array}$ & & \\
\hline 15 & Tan to medium gr & \multirow{14}{*}{$\begin{array}{l}\text { Rounded } \\
\text { Irregular to cubical } \\
\text { Rounded } \\
\text { Cubical } \\
\text { Irregular to cubical } \\
\text { Cubical } \\
\text { Rounded } \\
\text { Cubical }\end{array}$} & \\
\hline 16 & Reddish b & & \\
\hline 17 & & & \\
\hline 18 & Dark brown. & & \\
\hline 20 & gray, some tan. & & \\
\hline 21 & Dar & & \\
\hline 22 & Dark gray to brown. & & \\
\hline 23 & $\mathrm{Ta}$ & & \\
\hline 24 & $\mathrm{R}$ & & \\
\hline 25 & $\mathrm{~B}_{1}$ & & \\
\hline 26 & M & & \\
\hline 27 & $\mathrm{Re}$ & & \\
\hline 28 & to brown. & & \\
\hline & & & \\
\hline
\end{tabular}

1 "Rounded" particles are approximately spherical or egg-shaped. "Cubical" particles are approximately equal in all three dimensions but not rounded. "Irregular" particles are other than rounded or cubical.

${ }_{2}$ "Rough" texture means that the cellular interior is at least partly exposed.
The gage point inserts used in this investigation were No. 12-24 stainless steel screws with a No. 59 drill-hole drilled in the end. The method of holding the insert in place on the mold during casting is shown in figure 21 . The brass holding screw was removed when the concrete was removed from the mold.

Upon removal from the mold each gage point was cleaned and the gage holes were lightly reamed with a $60^{\circ}$ reamer until the pointed legs of the measuring gage could be well seated.

Since the No. 12-24 screw is no longer considered a stock size it was thought that another size screw should be used for future investigations. Also the standard nut used at the end of the insert did not provide sufficient thread length for foolproof use. At the present time a brass knurled nut with twice the thread length is being used.

\subsection{Steam Curing of the Concrete}

The steam-curing chamber was a fairly wellinsulated room approximately 6 - by 7 - by 6 -ft. The walls and ceiling of the room were lined with heavy aluminum foil. A 1-in. concrete overlay on the floor had been placed over a vapor barrier.

The steam source was a 2-stage generator as sold for use in steam rooms of health clubs. The generator was heated with two separate $4 \mathrm{kw}$ electric units which were individually thermostatically controlled. One unit, called the maintenance heater, was on all the time so that there was a continuous flow of steam into the curing chamber. The second $4 \mathrm{kw}$ unit was used to increase the steam flow until the preset temperature was reached.

The steam was fed into the chamber through 1/2-in. copper tubing in which $1 / 8$-in. holes were drilled for distribution of steam.

The two stages of the generator were controlled by timers so that the rate of temperature rise as well as the curing period could be automatically controlled. Figure 22 shows a typical curing

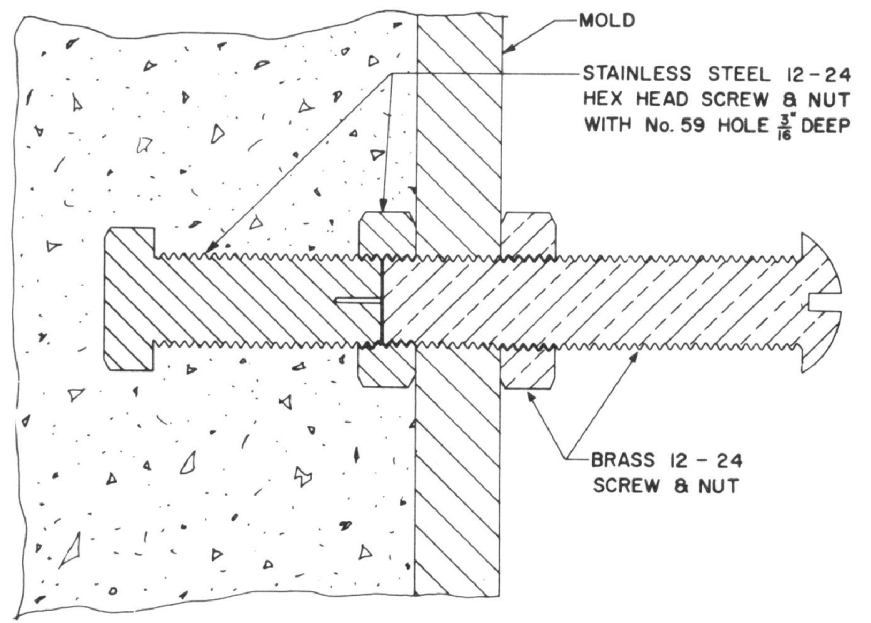

FIgURE 21. Stainless-steel Whittemore gage point used in the creep and shrinkage tests.

The brass screw and nut were used to maintain the position of the gage point until the concrete hardened. 


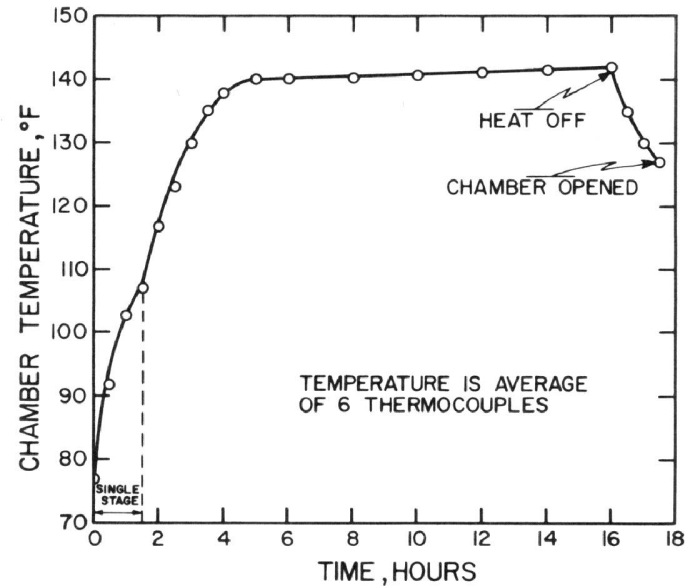

FIgURE 22. Typical steam-curing cycle for concretes made with type III cement.

The first heating stage of the steam generator was on for about $1 \frac{1}{2} \mathrm{hr}$ before the second stage was turned on. The concrete was allowed to set about $4 \mathrm{hr}$ before steam curing.

cycle in the steam chamber. Concretes made with type III cement were cured for $12 \mathrm{hr}$ at $140^{\circ} \mathrm{F}$, while those made with type I cement were cured $36 \mathrm{hr}$ at $140{ }^{\circ} \mathrm{F}$.

\subsection{Preparation of Cylinder Bearing Surfaces}

In lieu of the usual capping procedure, all creep and strength specimens were prepared for loading by wet-grinding their ends flat and parallel in a vertical-spindle surface grinder. The concrete specimens were held to the 36 -in. rotary magnetic chuck by a special steel jig which also assured that the specimens ends were ground perpendicular to their axes. Figure 23 shows the grinder with the holding jig and specimens in grinding position.

The grinding was done as soon as possible following removal of the specimens from the mold. It took approximately 15 min to grind both ends of a set of four specimens.

A short preliminary test was made to determine if there would be any obvious difference in the compressive strength due to the difference in the bearing surfaces of ground and capped specimens. Thirty-one 6 - by 12 -in. cylinders were cast from a single batch of sand and gravel concrete. The specimens were all steam-cured $12 \mathrm{hr}$ at $140{ }^{\circ} \mathrm{F}$ and then air-dried until tested. The bearing surfaces of 20 cylinders were prepared by grinding and the other 11 were capped with a standard sulfur-silica capping compound. The grinding was done when the concrete was 24 to $26 \mathrm{hr}$ old and the capping the day before testing.

Eleven specimens prepared by each method were loaded in compression at $80,000 \mathrm{lb} / \mathrm{min}$ until failure in a hydraulic testing machine when 3 days old. The results are listed in table 17. From these data it would seem that grinding the ends of the specimen may have a slight advantage over the capping. The coefficient of variation $(V)$ was computed as 4.5 percent for the capped cylinders and 2.6 percent for the ground cylinders.

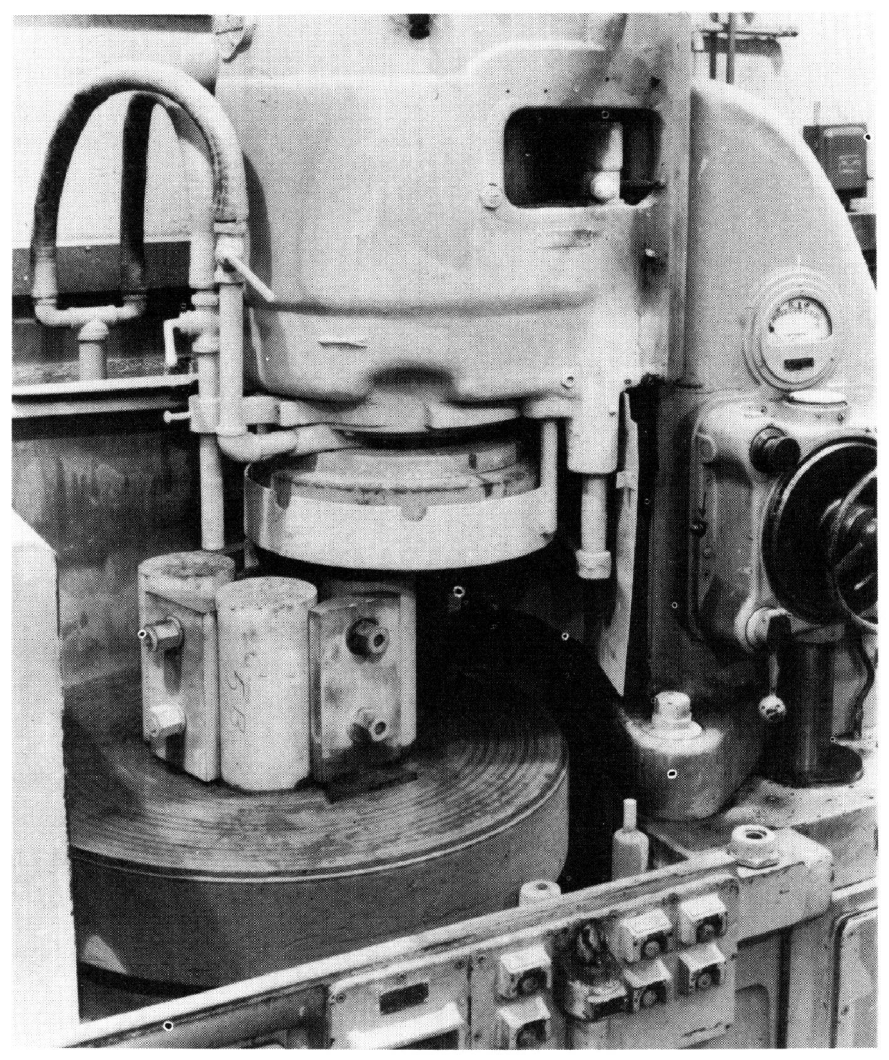

FIGURE 23. Method of holding and grinding the bearing surfaces of four 6-by 12-in. cylinders.

Both ends of each cylinder were ground. The height of the cylinders after grinding averaged about 11.86 in. A C-80 grit stone was used in the grinder.

TABLE 17. Effect of capping method on the compressive strength test results

\begin{tabular}{|c|c|c|}
\hline \multirow{2}{*}{ Cylinder } & \multicolumn{2}{|c|}{ Breaking loads } \\
\hline & Ground ends & Capped ends \\
\hline${ }^{1}{ }^{1}{ }^{2}$ & $\begin{array}{l}l b \\
111,400 \\
107,100 \\
104,500 \\
106,300 \\
108,100 \\
111,100 \\
110,400 \\
105,000 \\
106,600 \\
110,200 \\
104,200\end{array}$ & $\begin{array}{r}l b \\
102,000 \\
110,900 \\
106,600 \\
92,500 \\
108,800 \\
104,800 \\
107,500 \\
106,700 \\
104,500 \\
108,700 \\
111,400\end{array}$ \\
\hline $\begin{array}{l}\text { Avg. load, lb. } \\
\text { Avg. strength, psi }\end{array}$ & $\begin{array}{r}107,700 \\
3,810\end{array}$ & $\begin{array}{r}105,700 \\
3,750\end{array}$ \\
\hline
\end{tabular}

\subsection{Rate of Loading for Compressive Strength Test}

In the compressive strength tests of the cylinders for this investigation two different rates of loading were used. The lowest rate, about $22 \mathrm{psi} / \mathrm{sec}$, was adopted as the maximum rate at which stressstrain data could be reliably determined "on-therun". As the sample size for each concrete at each age was two cylinders, the second cylinder could be tested at some other rate for comparison. 
ASTM method C39-61 specifies that the rate of loading for the compressive test should be within the range of 20 to $50 \mathrm{psi} / \mathrm{sec}$. It was decided that the second cylinder of each set would be loaded at double the first, or $44 \mathrm{psi} / \mathrm{sec}$. Both rates are within the ASTM specification.

An analysis of 295 sets of cylinders indicate that the faster rate of loading increased the measured compressive strength only 0.2 percent over the slow rate. This increase which is insignificant when compared with the within-test coefficient of variation of 3.6 percent would seem to show that within the allowable ASTM rates the rate of loading will have no effect on the measured compressive strength values.

\subsection{Creep Test}

\subsubsection{Creep Test Frame}

All creep test frames used in this investigation were essentially as used by Shideler [1] and as shown in figure 24 . The $7 / 8$-in. rods for most of the frames were made from an AISI C-1040 Steel with a yield point of $87,000 \mathrm{psi}$. The double nuts over

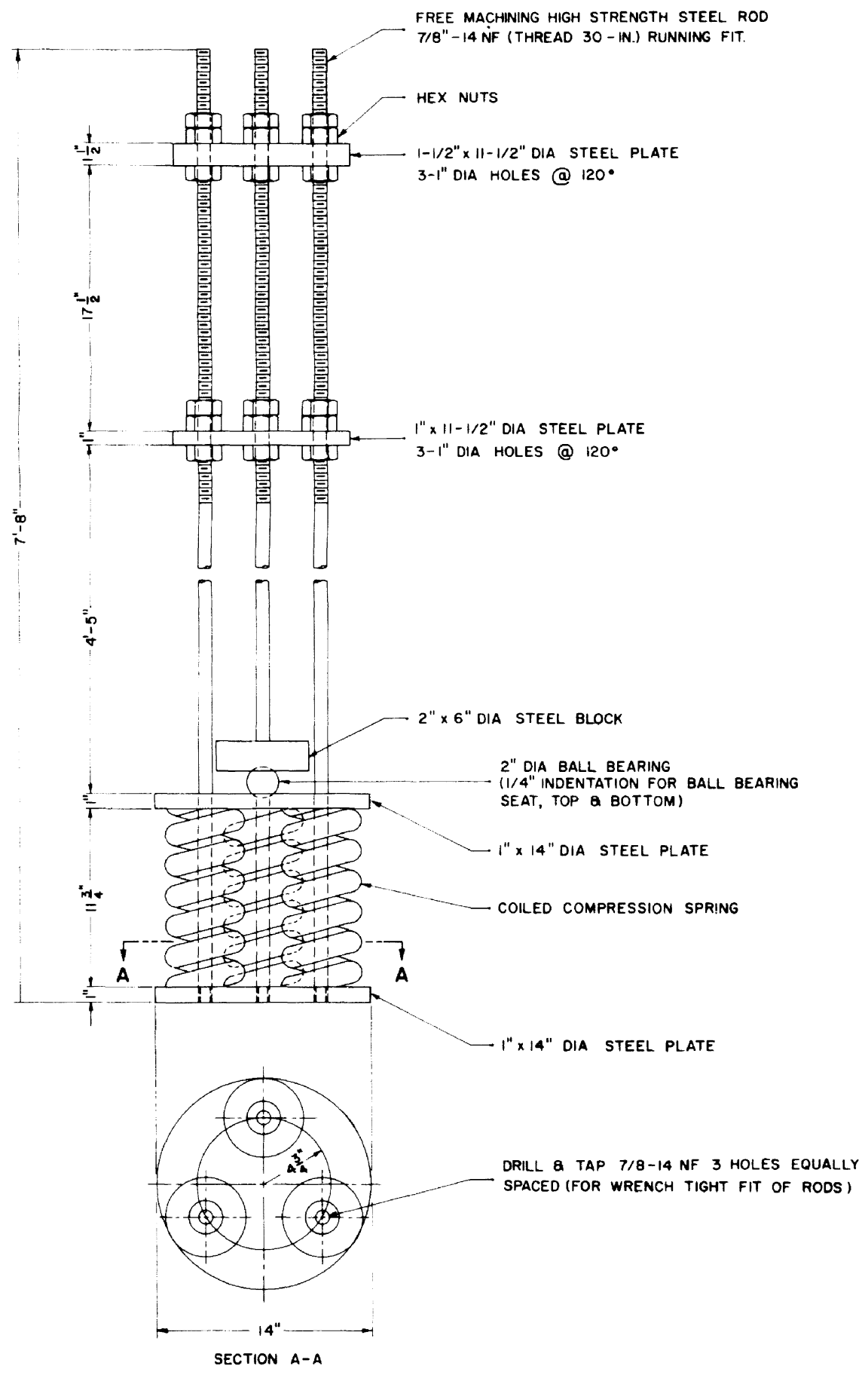

FIGURE 24. Construction details of creep frame used for the 2000 psi load on thrce 6-by 12-in. cylinders. 
the top plates were thought to be necessary for the higher-load frames as the threads on the rods had been made for a loose running fit of the nuts. All plates were torch cut to size. The springs were designed so that the deflection at the expected load would be approximately 2 -in.

\subsubsection{Loading Procedure}

When the investigation was being planned, it had been decided to limit the variation in strain due to eccentricity of the load to 10 percent. It had been thought that the eccentricity might be easily determined by measuring the strain in each rod as the load was applied. It was found that this procedure could not be used as the strains in the three rods were about equal even when the eccentricity was rather high. The eccentricity was defined as the ratio of strain in the gage line with the highest strain to that with the lowest. There were some indications that factors other than load eccentricity contributed to the differences in strain.

It was found quite early that much time and effort would be saved if considerable care were taken in setting up each frame prior to loading. The frame was leveled on the floor so that the rods were as nearly vertical as could be determined. The top two plates were adjusted so that they were parallel to each other and perpendicular to the rods.

Two special jigs were used in setting up the test frames. One jig was used to make the springs symmetrical and concentric with the ball bearing seat in the plate. The other jig was used in checking that the test specimens were centered within the three rods at the top of the stack.

After the stack of three test cylinders and the two dummy cylinders were placed and centered in the frame, a small load (about 200 psi) was applied. Variation in the strain around the top test specimen was then determined. If the loading was judged to be more than 10 percent ecrentric the load was removed, and the top test specimen was rotated slightly relative to the other specimens. When the loading was within the 10 percent eccentricity, the load was increased to about $1 / 2$ the creep load (usually $1000 \mathrm{psi}$ ) and the eccentricity was determined as before. If the eccentricity was within acceptable linits at this load, the final load increment was applied.

The determination of the actual load transfered to the frame from the hydraulic ram presents experimental difficulties with spring-loaded test frames. The usual procedure in transferring the load from the hydraulic ram to the three reaction rods is to tighten the nuts while the ram carries the entire creep load. However, the degree of tightening. which is difficult to evaluate, determines the loss of the applied load upon the release of the ram. Accordingly, for this investigation bonded wire strain gages were attuched to the rods for their use as dynamometers in transferring the load. In effect, these dynamometers were calibrated every time the frame was loaded by measuring the strain in the rod when the required creep load was applied to the specimens by the ram.

\subsubsection{Effect of Eccentricity of Loading on Creep Measurements}

The eccentricity of loading could be determined and controlled at the initial loading, but this control was not possible when the needed adjustments of the applied load were made. For this reason it was assumed that, although the eccentricity probably changed slightly each time a frame was reloaded, the average long-term eccentricity could be determined by comparing the total deformations measured in each gage line. It was thought that through a comparison of the results from the two companion specimens the effect of eccentricity on the values of creep could be determined. An analysis was made, and it was concluded that if load eccentricity had any effect on the measured creep values, it was masked by the effect of other factors.

The eccentricity of loading caused an average variation of strain in the three gage lines of \pm 7 percent; the individual strains differed from the mean by 0 to 16 percent.

\subsubsection{Effect of Loading Time on Creep Measurements}

The measured change in length of the creep specimen obtained immediately after the initial application of load is usually considered to be elustic movement. However, this strain can include some creep strain if the period of time from initial loading until measurements are made is considerable. Thus, the elastic strain for the last specimen in a given frame to be measured would include more creep strain than that for the first specimen measured. Another factor affecting this measurement would be the time required to make adjustments due to eccentricity of loading.

As a check on the extent of the creep strain which might be included in the elastic strain, a comparison was made between the stress-strain dat a for the compressive test specimen and the companion creep specimens. This comparison was made by comparing the strains at the same stress level for both methods of loading. It was assumed that on the avernge, results from the 64 batches of concrete considered, the effect of individual differences between companion specimens would be cincelled out, and only differences due to the different methods of loading would affect the results.

The results indicated that, on the average, the measured elastic strain in the creep specimens was 4 percent greater than the strain in the companion compressive test specimen. This result means that, on the average, 4 percent of the initial strain which was thought to be elastic was probably creep. 


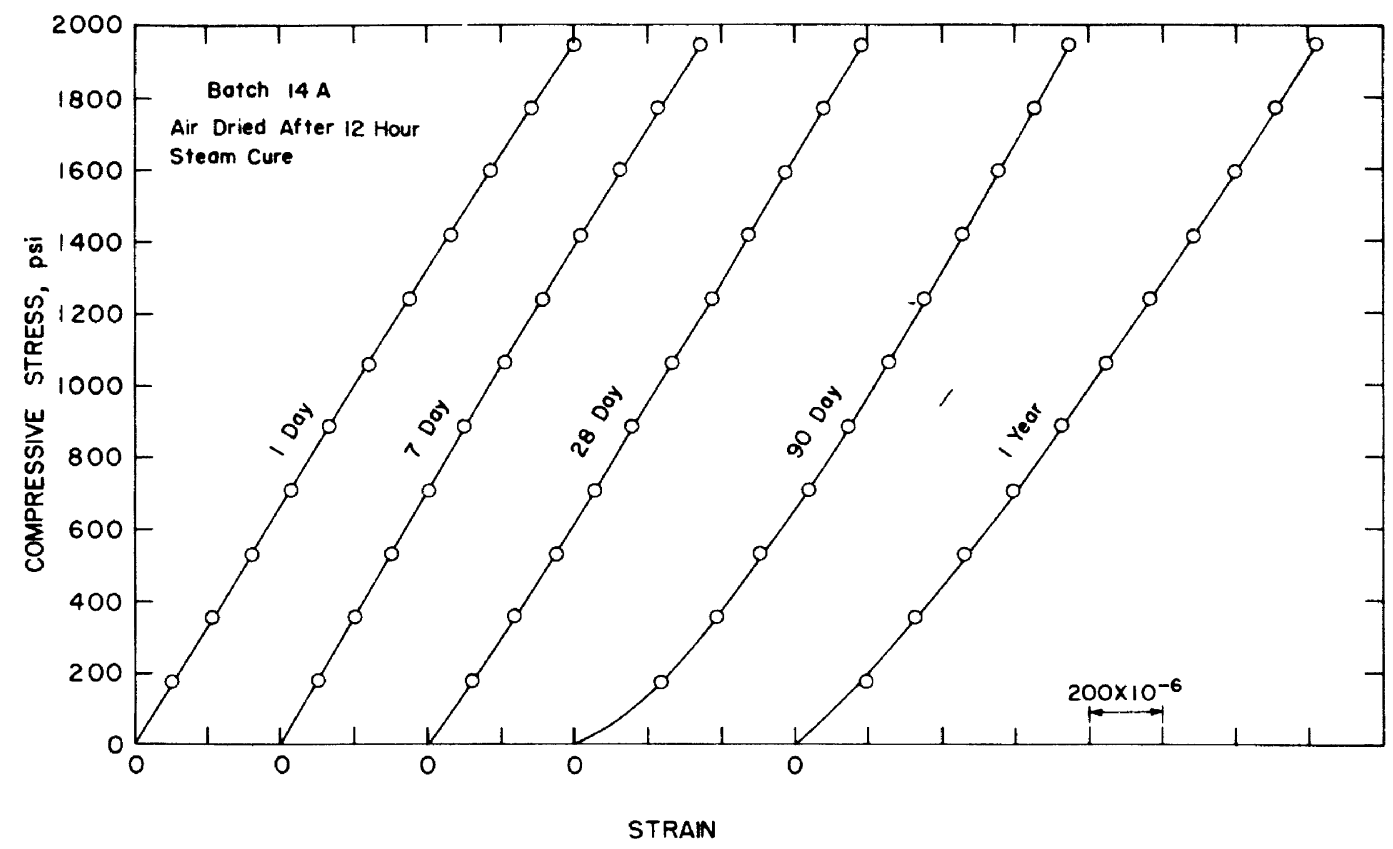

FIGURE 25. Stress-strain data for a concrete made with aggregate 14.

\subsection{Peculiarities of an Anomalous Lightweight Aggregate}

When the series A concretes made from aggregate no. 14 were about three weeks old, it was noticed that the concretes were considerably different than the others. For instance, the measured drying shrinkage was less than expected. This observation led to the discovery of surface shrinkage racks on all drying specimens of these concretes. These cracks were visible with the naked eye even on the loaded specimens.

The 28 day flexural test revealed a remarkable decrease in modulus of rupture for the drying specimens. (See fig. 1 and table 5.)

When the concrete was 90 days old, the cracks had progressed to such an extent that the resonantfrequency determinations for the dynamic test were meaningless, and the strain readings at low stress levels for the stress-strain test were such that the stress-strain curve was concave upwards. The effect of these cracks on the stress-strain curve can be seen by comparing the typical stressstrain curves of figure 2 and the curves for aggregate 14 concrete shown in figure 25 . It can be seen that this effect is the greatest for the 90-day test.

Since the shrinkage stresses were being relieved by cracking, the measurements continued to indicate low shrinkage values for this concrete until the 1-year readings. A comparison of the shrinkage-time values for the aggregate 14 concrete with the average lightweight is shown in figure 26 .

The shrinkage cracks were still visible on the unloaded 6 - by 12 -in. cylinders when 1 year old, but were not visible on the loaded specimens.

The shrinkage cracking affected the shrinkage, flexural strength, and stress-strain determinations,

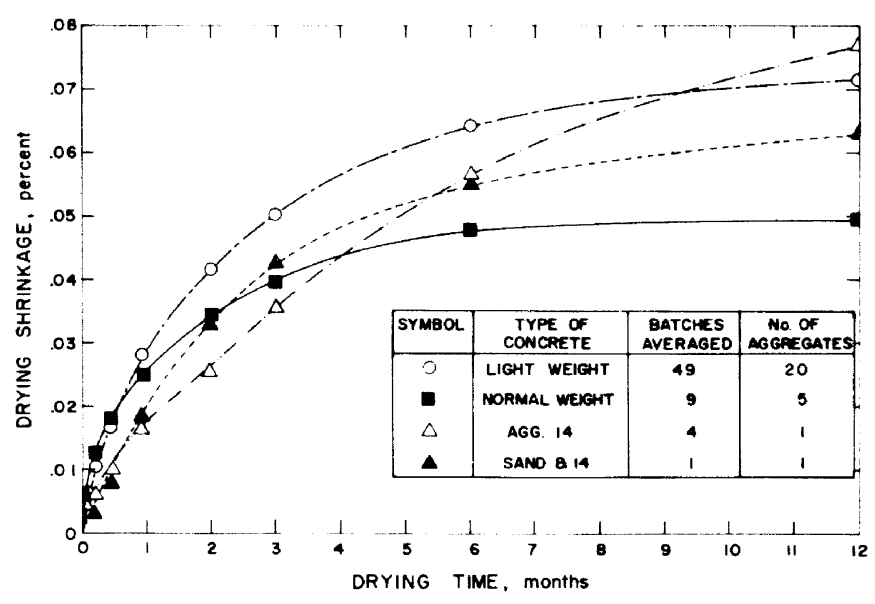

Figure 26. Comparison of shrinkage-time data for steamcured concretes made with aggregate 14 and with other aggregates.

but did not appear to effect the compressive strength.

When this cracking was noticed, specimens of the other concretes were examined for evidence of cracking. However, no sign of cracking could be observed on any other concrete.

Shrinkage cracking in the surface of concrete specimens of various sizes have been noted by Grieb and Werner [23]; and Kennedy [23] in his discussion of this work has reported measuring the buildup of a compressive stress exceeding 120 psi in the center of a drying 30- by 60 -in. concrete cylinder. The extent of the surface cracking which 'might be expected in a concrete is probably affected greatly by the size of the specimen and the drying conditions.

It should be emphasized that this particular aggregate (No. 14) is usually produced only in the coarse fraction which is intended to be used 
with natural sand in place of the lightweight fines used in making the series A concrete. Shrinkage-time values for a concrete made from the coarse fraction of this aggregate and a natural sand for test $\mathrm{V}$ of series $\mathrm{B}$ are included in figure 26 for comparison. This semilightweight concrete did not exhibit the unusual behavior noted above for the all-lightweight concretes.

\section{References}

[1] Shideler, J. J., Lightweight aggregate concrete for structural use, ACI Journal, Proc. 54, 299-328 (Oct. 1957).

[2] Shideler, J. J., Manufacture and use of lightweight aggregates for structural concrete, PCA Research and Development Laboratory Bull. D 40.

[3] Best, C. H., and Polivka, M., Creep of lightweight concrete, Mag. Concrete Res., 11, No. 33, 129-134 (Nov. 1959).

[4] Jones, Truman R., Hirsch, T. J., and Stephanson, Henson K., The physical properties of structural quality lightweight aggregate concrete, Final Report from Research Project $\mathrm{RP}-7$ for the Texas Highway Dept.. Texas Transportation Institute, Texas A. and M. College System (Aug. 1959).

[5] Hanson, J. A., Tensile strength and diagonal tension resistance of structural lightweight concrete, ACI Journal, Proc. 58, 1-37 (July 1961).

[6] Abrams, Duff A., Flexural strength of plain concrete, ACI Journal Proc. 18, 20-45 (1922).

[7] Walker, Stanton and Bloem, Delar L., Studies of flexural strength of concrete-Part 3: Effects of variations in testing procedures, Proc. ASTM 57, 1122-1142 (1957).

[8] Glucklich, Joseph, and Ishai, Ori, Creep mechanism in cement mortar, ACI Journal, Proc. 59, 923-948 (July 1962).

[9] Verbeck, G. J., Carbonation of hydrated portland cement, ASTM Special Publication No. 205, 1736 (Feb. 1958).
[10] Neville, A. M., Role of cement in the creep of mortar, ACI Journal, Proc. 55, 963-983 (March 1959).

[11] Hanson, J. A., A 10-year study of creep properties of concrete, Concrete Laboratory Report No. SP-38, U.S. Bur. of Reclamation (1953).

[12] Lyse, Inge, The shrinkage and creep of concrete, Mag. Concrete Res., 11, No. 33, 143-150 (Nov. $1959)$.

[13] Recommended practice for evaluation of compressive test results of field concrete, ACI Journal, Proc. 54, 1-9 (July 1957).

[14] Price, Walter H., Factors influencing concrete strength, ACI Journal, Proc. 47, 417-432 (Feb. 1951).

[15] Hanson, J. A., Optimum steam curing procedure in precasting plants, ACI Journal, Proc. 60, 75-99 (Jan. 1963).

[16] Higginson, Elmo C., Effect of steam curing on the important properties of concrete, ACI Journal, Proc. 58, 281-296 (Sept. 1961).

[17] Pauw, Adrian, Static modulus of elasticity of concrete as affected by density, ACI Journal, Proc. 57, 679-687 (Dec. 1960).

[18] Kingham, R. I., Fisher, J. W., Viest, I. M., Creep and shrinkage of concrete in outdoor exposure and relaxation of prestressing steel, Highway Res. Board Special Report $66^{6}$ (Publication 933), 103131 (1961).

[19] Corley, W. G., Sozen, M. A., Siess, C. P., Time dependent deflections of prestressed concrete beams, H RB Bull. 307 (Publication 939), 1-25 (1961).

[20] Freudenthal, A. M., and Roll, F., Creep and creep recovery of conerete under high compressive stress, ACI Proc. 54, 111-1142 (Jume 1958).

[21] Mellenry, I), A new aspect of creep in concrete and its application to design, ASTM 43, 1069-1084 (1943).

[22] Carlson, R. W., Drying shrinkage of concrete as affected by many factors, Proc. ASTM 38, 419437, Part İ (1938).

[23] Grieb, W. E., and Werner, George, Comparison of splitting tensile strength of concrete with flexural and compressive strengths, Proc. ASTM 62, 972975 (1962). Author's Closure and Discussion by T. B. Kennedy. 


\section{THE NATIONAL BUREAU OF STANDARDS}

The scope of activities of the National Bureau of Btandards at its major laboratories in Washington, D.C., and Boulder, Colorado, is suggested in the following listing of the divisions and sections engaged in technical work. In general, each section carries out specialized research, development, and engineering in the field indicated by its title. A brief description of the activities, and of the resultant publications, appears on the inside of the front cover.

\section{WASHINGTON, D.C.}

Electrieity. Resistance and Reactance. Electrochemistry. Electrical Instrumente. Magnetic Measurements. Dielectrics. High Voltage. Absolute Electrical Measurements.

Metrology. Photometry and Colorimetry. Refractometry. Photographic Research. Length. Engineering Metrology. Mass and Volume.

Heat. Temperature Physics. Heat Measurements. Cryogenic Physics. Equation of State. Statistical Physics.

Radiation Physics. X-ray. Radioactivity. Radiation Theory. High Energy Radiation. Radiological Equipment. Nucleonic Instrumentation. Neutron Physics.

Analytical and Inorganic Chemistry. Pure Substances. Spectrochemistry. Solution Chemistry. Standard Reference Materials. Applied Analytical Research. Crystal Chemistry.

Mechanics. Sound. Pressure and Vacuum. Fluid Mechanics. Engineering Mechanics. Rheology. Combustion Controls.

Polymers. Macromolecules. Synthesis and Structure. Polymer Chemistry. Polymer Physics. Polymer Characterization. Polymer Evaluation and Testing. Applied Polymer Standards and Research. Dental Research.

Metallurgy. Engineering Metallurgy. Metal Reactions. Metal Physics. Electrolysis and Metal Deposition.

Inorganic Solids. Engineering Ceramics. Glass. Solid State Chemistry. Crystal Growth. Physical Properties.

Crystallography.

Building Research. Structural Engineering. Fire Research. Mechanical Systems. Organic Building Materials. Codes and Safety Standards. Heat Transfer. Inorganic Building Materials. Metallic Building Materials.

Applied Mathematics. Numerical Analysis. Computation. Statistical Engineering. Mathematical Physics. Operations Research.

Data Processing Systems. Components and Techniques. Computer Technology. Measurements Automation. Engineering Applications. Systems Analysis.

Atomic Physics. Spectroscopy. Infrared Spectroscopy. Far Ultraviolet Physics. Solid State Physics. Electron Physics. Atomic Physics. Plasma Spectroscopy:

Instrumentation. Engineering Electronics. Electron Devices. Electronic Instrumentation. Mechanical Instruments. Basic Instrumentation.

Physical Chemistry. Thermochemistry. Surface Chemistry. Organic Chemistry. Molecular Bpectroscopy. Elementary Processes. Mass Spectrometry. Photochemistry and Radiation Chemistry.

Ofice of Weights and Measures.

BOULDER, COLO.

Cryogenic Engineering Laboratory. Cryogenic Processes. Cryogenic Properties of Bolids. Cryogenic Technical Bervices. Properties of Cryogenic Fluids.

\section{Central Radio pROPAgation LABORATORY}

Ionosphere Research and Propagation. Low Frequency and Very Low Frequency Research. Ionosphere Research. Prediction Services. Sun-Earth Relationships. Field Engineering. Radio Warning Services. Vertical Soundings Research.

Troposphere and Space Telecommunications. Data Reduction Instrumentation. Radio Noise. Tropospheric Measurements. Tropospheric Analysis. Spectrum Utilization Research. Radio-Meteorology. Lower Atmosphere Physics.

Radio Systems. Applied Electromagnetic Theory. High Frequency and Very High Frequency Research. Frequency Utilization. Modulation Research. Antenna Research. Radiodetermination.

Upper Atmosphere and Space Physics. Upper Atmosphere and Plasma Physics. High Latitude Ionosphere Physics. Ionosphere and Exosphere Scatter. Airglow and Aurora. Ionospheric Radio Astronomy.

\section{RADIO STANDARDS LABORATORY}

Radio Standards Physics. Frequency and Time Disseminations. Radio and Microwave Materials. Atomic Frequency and Time-Interval Standards. Radio Plasma. Microwave Physics.

Radio Standards Engineering. High Frequency Electrical Standards. High Frequency Calibration Services. High Frequency Impedance Standards. Microwave Calibration Services. Microwave Circuit Standards. Low Frequency Calibration Servioes.

\section{NBS LABORATORY ASTROPHYSICS GROUP}

(Joint Institute for Laboratory Astrophysics at University of Colorado) 
